Historic, archived document

Do not assume content reflects current scientific knowledge, policies, or practices. 



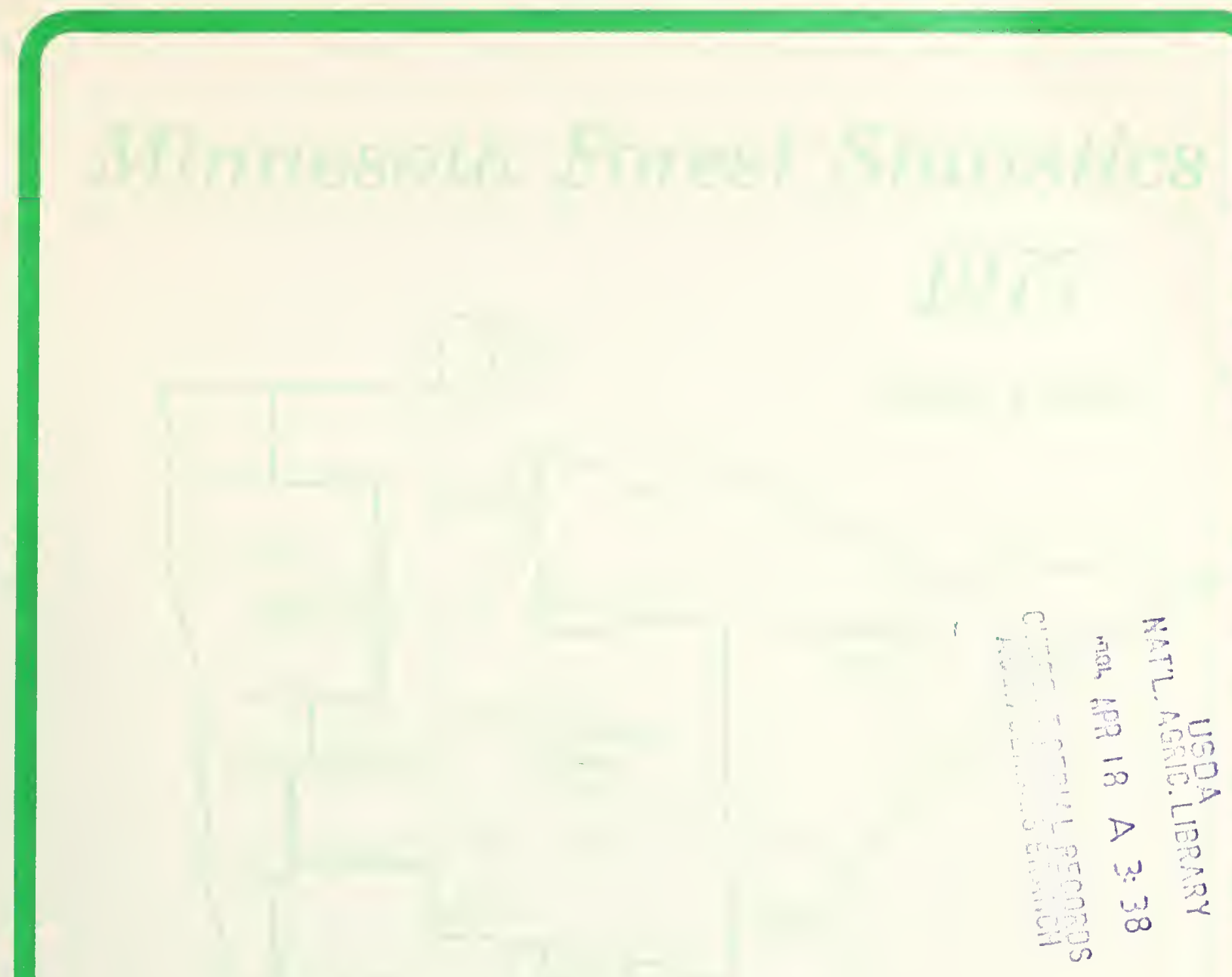

North Central Forest Experiment Station

Forest Service, U.S. Department of Agriculture
LIBRARY

FORESTRY SCIENCES LABORATORY

P. O. BOX 909

JUNEAU, ALASKA 99801 
North Central Forest Experiment Station Forest Service-U.S. Department of Agriculture 1992 Folwell Avenue St. Paul, Minnesota 55108

Manuscript approved for publication April 15, 1980 


\section{FOREWORD}

Resources Evaluation (formerly called Forest Survey) is a continuing endeavor as mandated by the Forest and Rangeland Renewable Resources Planning Act of 1974, which was preceded by the McSweeney-McNary Forest Research Act of 1928. Its objective is to periodically inventory the Nation's forest land to determine its extent, condition, and volume of timber, growth, and depletions. This kind of up-to-date information is essential to frame intelligent forest policies and programs. USDA Forest Service regional experiment stations are responsible for conducting these inventories and publishing summary reports for individual States. The North Central Forest Experiment Station is responsible for Resources Evaluation work done in Michigan, Wisconsin, Minnesota, North Dakota, eastern South Dakota, Nebraska, Iowa, Illinois, Indiana, Missouri, and Kansas.

Fieldwork for the 1977 Minnesota Forest Survey was begun in July 1974 and was completed in July 1978. Reports on the three previous surveys of Minnesota's timber resource are dated 1936,1953 , and 1962.

Similar Resource Bulletins reporting statistical highlights and detailed tables on the timber resource of the four Survey Units in Minnesota (see cover) are available.

More accurate survey information was obtained during the 1977 survey than otherwise would have been feasible because of intensified field sampling. This was made possible by extra funding and manpower provided the North Central Station by the State Legislature through the Minnesota Department of Natural Resources. The Department also assisted in a canvass of primąry wood-using plants in the State, which was used to help estimate the quantity of timber products harvested in Minnesota.

Aerial photos used in the Minnesota Forest Inventory were furnished by the Boise Cascade Company, Chippewa National Forest, Lake County Land Commissioner's Office, Minnesota Department of Natural Resources, Superior National Forest, USDA Agricultural Stabilization and Conservation Service, and USDI Bureau of Indian Affairs.

The following North Central Forest Experiment Station office personnel helped prepare the State tables and manuscript:

Mary Jean Hanson, Secretary

Patrick Peine, Statistical assistant

Gerhard K. Raile, Associate mensurationist

Carol Weist, Computer programmer 


\section{CONTENTS}

Principal Tree Species Groups in Minnesota ..........

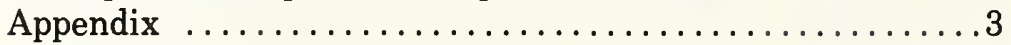

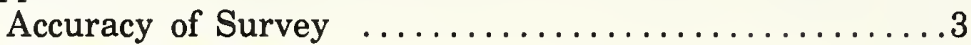

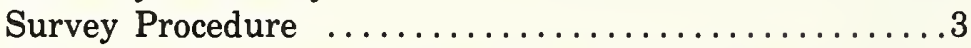

Comparing Minnesota's Fourth Survey with the

Third Survey $\ldots \ldots \ldots \ldots \ldots \ldots \ldots \ldots \ldots \ldots \ldots$

Metric Equivalents of Units Used in this Report ...5

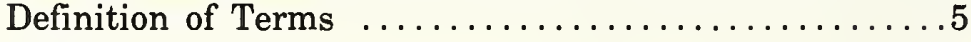

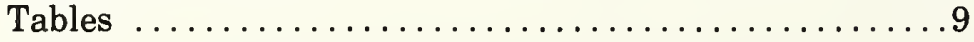




\title{
MINNESOTA FOREST STATISTICS, 1977
}

\author{
Pamela J. Jakes \\ Associate Resource Analyst
}

\section{HIGHLIGHTS}

\section{Area}

- Commercial forest area declined from 15.4 million acres in 1962 to 13.7 million acres in 1977, an annual rate of change of -0.8 percent.

- Commercial forest area is concentrated in the Northern Pine (5.7 million acres) and AspenBirch (5.5 million acres) Forest Survey Units.

- Farmers own the largest portion of Minnesota's commercial forest land base (3.4 million acres), followed by the State of Minnesota (2.7 million acres) and county and municipal agencies (2.3 million acres).

- Aspen is the most common forest type in Minnesota, occurring on 5.3 million acres of commercial forest land.

- The majority of the commercial forest land (6.9 million acres) is in poletimber stands. The remaining commercial forest area is fairly evenly divided among sawtimber, and seedling and sapling stands.

- The area of productive-reserved forest land increased from 470,100 acres in 1962 to 1.2 million acres in 1977, due largely to creation of Voyageur's National Park and expansion of wilderness portions of the Boundary Waters Canoe Area.
- Eleven percent of Minnesota's forest land is unproductive.

\section{Volume}

- In 1977 there were 12.9 billion cubic feet of timber in Minnesota-11.4 billion cubic feet in growing-stock trees, 1.4 billion cubic feet in rough and rotten trees, and 0.1 billion cubic feet in salvable dead trees.

- Growing-stock volume per acre on commercial forest land increased from 613 cubic feet in 1962 to 836 cubic feet in 1977, resulting in a 21 percent increase in growing-stock volume, from 9.4 to 11.4 billion cubic feet.

- Sawtimber volume increased 65 percent between surveys, from 14.9 billion board feet in 1962 to 24.6 billion board feet in 1977. This reflects the increasing maturity of Minnesota's forests.

- Growing-stock volume is concentrated in the Northern Pine (5.0 billion cubic feet) and AspenBirch (4.5 billion cubic feet) Forest Survey Units.

- Aspen accounts for 30 percent of the growingstock volume ( 3.4 billion cubic feet) and 24 percent of the sawtimber volume ( 6.0 billion board feet).

- Farmers own 2.7 billion cubic feet of growingstock volume, county and municipal agencies own 2.0 billion cubic feet, and the State owns 1.9 billion cubic feet. 


\section{Stand Conditions}

- Net annual growth of growing stock for softwoods increased from 106.8 million cubic feet in 1961 to 119.8 million cubic feet in 1976 , but net annual growth for hardwoods decreased from 257.4 million cubic feet to 229.1 million cubic feet resulting in an overall decrease in net annual growth between surveys.

- Net annual growth rate of growing stock is 3.0 percent of inventory. The growth rate for softwoods ( 3.4 percent) is higher than the growth rate for hardwoods (2.9 percent).

- Net annual growth per acre remained fairly constant between surveys-24 cubic feet in 1961 compared to 25 cubic feet in 1976.

- Mortality of growing-stock trees averaged 1.2 percent of inventory.

- Disease is the most common cause of mortality and accounts for 57 percent of 141.5 million cubic feet.

- Sixty-five percent of Minnesota's commercial forest land is capable of growing trees at least 51 feet high at age 50 .

- More than two-thirds of Minnesota's sawtimber volume is in butt log grade 3 timber.

- Fifty-seven percent of the commercial forest area is in stands less than 51 years old.

\section{Timber Use}

- Timber removals from growing stock totaled 193.6 million cubic feet in 1976-135.0 million cubic feet for timber products, $\mathbf{5 3 . 5}$ million cubic feet for other removals, and 5.1 million cubic feet for logging residue.

- Growing-stock removals in 1976 are 33 percent higher than removals in 1962. The increase is entirely due to greater hardwood removals.

- Fifty-four percent of growing-stock removals came from farmer-owned and miscellaneous private-owned land.

- Timber products output in 1975 totaled 169.9 million cubic feet-63 percent as pulpwood, 17 percent as saw logs, and 14 percent as fuelwood.

- Primary plant residue in 1975 totaled 11.6 million cubic feet-7.1 million cubic feet from hardwoods and 4.5 million cubic feet from softwoods. An additional 5.6 million cubic feet of residue was bark.

\section{PRINCIPAL TREE SPECIES GROUPS IN MINNESOTA ${ }^{1}$}

\section{Softwoods:}

Eastern white pine ............Pinus strobus Red pine ..................Pinus resinosa Jack pine .................Pinus banksiana Black spruce ................Picea mariana White spruce ................Picea glauca

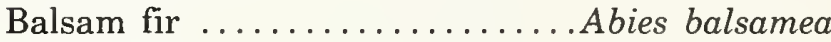
Tamarack .................... Larix laricina Northern white-cedar ........Thuja occidentalis Other softwoods:

Eastern redcedar .......Juniperus virginiana Scotch pine ..............Pinus sylvestris

\section{Hardwoods:}

White oaks:

White oak.............. Quercus alba Bur oak ............. Quercus macrocarpa Swamp white oak ......... Quercus bicolor Select red oak:

Northern red oak ..........Quercus rubra

Other red oaks:

Black oak ..............Quercus velutina

Northern pin oak ......Quercus ellipsoidalis

Hickories:

Shagbark hickory ............. Carya ovata

Bitternut hickory .......... Carya cordiformis Yellow birch ...........Betula alleghaniensis Hard maples:

Sugar maple ..............Acer saccharum

Black maple .................Acer nigrum Soft maples:

Red maple .................Acer rubrum Silver maple .............Acer saccharinum Ashes:

White ash .............Fraxinus americana Black ash .................Fraxinus nigra Green ash ..........Fraxinus pennsylvanica Balsam poplar ...........Populus balsamifera Paper birch ............... Betula papyrifera

\footnotetext{
${ }^{1}$ The common and scientific names are based on "Check List of Native and Naturalized Trees of the United States (including Alaska)," by Elbert L. Little, Jr. 1979. U.S. Department of Agriculture, Agriculture Handbook 541, 375 p.
} 
Aspens:

Bigtooth aspen ........Populus grandidentata

Quaking aspen ..........Populus tremuloides

Basswood .................Tilia americana

Elms:

American elm ...........Ulmus americana

Slippery elm ...............Ulmus rubra

Rock elm ..............Ulmus thomassii

Select hardwoods:

Butternut .............Juglans cinerea
Black walnut ...........Juglans nigra

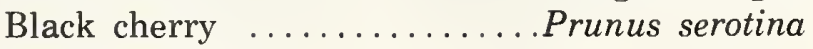

Other hardwoods:

Boxelder ..................Acer negundo

River birch ......................... nigra

Hackberry ................. oltis occidentalis

Eastern cottonwood ........Populus deltoides

Black willow .......................... nalix niga

Kentucky coffeetree .....Gymnocladus dioicus

\section{APPENDIX}

\section{Accuracy of Survey}

Resources Evaluation information is based on a sampling procedure designed to provide reliable statistics at the State and Survey Unit levels. Consequently, the reported figures are estimates only. However, a measure of reliability of these figures is given by sampling errors. These sampling errors mean that the chances are two out of three that if a 100 -percent inventory had been taken, using the same methods, the results would have been within the limits indicated.

For example, the estimated area of commercial forest land in Minnesota in 1977, 13,695.1 thousand acres, has a sampling error of \pm 0.39 percent $( \pm$ 53,900 acres). The commercial forest area from a $100-$ percent inventory, then, would be expected to fall between 13,749.0 and 13,641.2 thousand acres $(13,695.1 \pm 53.9)$, there being a one in three chance that this is not the case.

Sampling errors were calculated separately for National Forest land and other land because of the higher sampling intensity on this land. For example, the sampling error for growing-stock volume on land other than National Forest is \pm 0.82 percent but for Chippewa National Forest land it is \pm 7.47 percent.

The following tabulation shows the combined sampling errors for the 1977 Minnesota Forest Inventory:
Item

Minnesota totals $\begin{gathered}\text { Sampling } \\ \text { error } \\ \text { (percent) }\end{gathered}$

Growing stock:

Volume $\quad 11,454.0$ million cubic feet

Growth 348.9 million cubic feet

Removals 193.6 million cubic feet

5.33

Sawtimber:

Volume 24,607.2 million board feet

Growth 1,111.5 million board feet

Removals 460.3 million boardfeet

4.36

Commercial forest

land:

13,695.1 thousand acres

0.39

As survey data are broken down into sections smaller than State or Survey Unit totals, the sampling error increases. The smaller the breakdown, the larger the sampling error. For example, the sampling error for area of commercial forest land in a particular county is higher than that for total commercial forest area in the Survey Unit (table 70 shows the sampling errors for estimates smaller than State totals).

\section{Survey Procedure}

The major steps in the survey of Minnesota were as follows:

1. A total of 276,897 1-acre points were systematically distributed across aerial photos of the entire 
State, except the Chippewa and Superior National Forests. These points were classified as forest land $(72,700)$, unproductive forest land $(4,483)$, nonforest land $(197,674)$, or questionable $(2,040)$, in order to make a preliminary estimate of forest area. Next, all of the forest points $(72,700), 592$ of the unproductive forest points, and all of the questionable points $(2,040)$ were stereoclassified as to forest type, standsize class, and density. Then 9,796 points classed as forest, 592 points classed as unproductive, 276 points classed as questionable, and 25,498 points classified as nonforest were examined on the ground to correct the preliminary area estimate for errors in classification and for actual changes in land use since the photos were taken. At each of the 8,547 commercial forest locations, variable-radius plots (basal area factor 37.5 ) were established at 10 points uniformly placed over the sample acre. Tree measurements made at these locations were the basis for estimates of timber volume, growth, mortality, number of trees, and other forest classifications.

2. Growth and mortality on all commercial forest land were estimated using the Forest Resources Evaluation Program (FREP) ${ }^{2}$. This is an individual tree-growth projection system that uses stand and tree characteristics such as tree diameter, crown ratio, and stand density to estimate tree volumes.

3. Data from the National Forests were incorporated in the Survey data. Area statistics for the Chippewa National Forest were prepared by the Forest Timber Management staff from compartment examination records. In 1975, the Forest conducted its own forest inventory using 10-point variable-radius plots established by Forest personnel under the direction of the North Central Forest Experiment Station. Data from these plots were used as input for FREP, which, with modifications in the Program, was used to update volumes to 1977 . The updated Chippewa National Forest data were approved by the Forest and combined with Survey data from non-National Forest commercial forest land to estimate volume, growth, and mortality for the State.

Area statistics for the Superior National Forest were also prepared by the Forest Timber Management staff from compartment examination records. Volume data for Superior National Forest land were

\footnotetext{
${ }^{2}$ For more information on FREP, see: U.S. Department of Agriculture, Forest Service. 1979. A generalized forest growth projection system for the Lake States Region. U.S. Department of Agriculture Forest Service, General Technical Report NC-49, 96 p.
}

prepared by staff using permanent sample plots established by Forest personnel. Superior National Forest data, then, were added to data for the rest of the State computed from 10-point variable radius plot information.

4. Statistics on timber utilization during 1975 were obtained from mill surveys. The Minnesota Department of Natural Resources and the North Central Forest Experiment Station canvassed resident sawmills, veneer mills, and other primary wood-using plants. The North Central Forest Experiment Station canvassed resident pulpmills, as well as out-ofState sawmills, pulpmills, and veneer mills to determine their use of timber from Minnesota. Fuelwood and fencepost output was based on a sample of private landowners to determine their production of fuelwood and fenceposts, and on a canvass of industrial and public timber owners. Estimates of primary mill residue used for fuelwood were obtained from the canvass of Minnesota primary wood-using plants. Timber cut for products by ownership class was determined by a canvass of public and industrial timber owners. The portion of timber cut unaccounted for by the latter owners was grouped under "farmer and other owners".

5. A total of 1,028 felled trees were measured throughout the State during 1975-1976 to develop wood utilization factors for converting timber products output to timber removals for saw logs and pulpwood. Factors for veneer logs were obtained during the 1967-1968 Wisconsin utilization study. Factors for all other products were obtained during the 19601961 Minnesota utilization study.

\section{Field data were sent to St. Paul and compiled.}

\section{Comparing Minnesota's Fourth Survey with the Third Survey}

Data from new forest surveys are often compared with data from earlier ones to determine trends in forest areas and volumes. Changes in procedures and definitions between surveys make it necessary to adjust earlier survey data so they are comparable to data from the new survey.

The Unit boundaries in Minnesota changed between surveys so published 1962 commercial forest area was adjusted to take these changes into account. Then, a portion of the 1962 commercial forest area was withdrawn and added to unproductive forest (noncommercial) and to nonforest to allow for changes in the method of determining land uses. The 
resulting adjusted 1962 commercial area was then used to adjust 1962 inventory, net growth, and mortality volumes.

A test was made to ensure that it was possible to move from the adjusted 1962 volumes to the new 1977 volumes by means of Timber Resource Analysis System (TRAS), which is a Forest Service computer program for updating, backdating and projecting timber volume, growth, mortality, and removals. In order for the program to work, the 2 years to be reconciled must have comparable commercial forest land bases so that changes in volumes between surveys reflect actual changes in forest conditions or land use. To achieve this condition, it was necessary to make further adjustments in 1962 data so that land transferred from commercial forest to productive-reserved between the two surveys would not appear in the 1962 land base. If this adjustment were not made, removals between 1962 and 1977 would appear artificially high to absorb the loss of timber from this change in land status. This adjustment was made for the test only; area and volumes removed for the test were added back into the 1962 reported data.

TRAS recalculated 1962 volumes using 1977 estimates of cubic foot volume per tree and 1977 board foot-cubic foot ratios. This volume adjustment was necessary so that volume differences between surveys represented actual change and not merely change in the volume equations used on each occasion.

When the final adjustments of the 1962 data were completed, the resulting 1962 volumes and area were distributed among the four Survey Units. A check was made to ensure that it was possible to move from the adjusted 1962 volumes to the new 1977 volumes in each Unit. This was done using the average periodic difference between growth and removals for the two surveys and applying this difference to the 1962 volume. The same procedure was repeated for each individual species within each Unit to make sure inventory volumes reported for each species were consistent with reported growth and removals volumes.

Removals estimates for 1962 and 1977 are for total removals, including timber cut in addition to "other" removals, and are trend level removals. "Other" removals from transfer of commercial forest land to productive-reserved are not included. Previously published 1960 estimates are of timber cut only and do not include "other" removals. TRAS generated an estimate of 1962 "other" removals to provide the adjusted 1962 total removals.

\section{Metric Equivalents of Units Used in this Report}

1 acre $=4,046.86$ square meters or 0.405 hectare. 1,000 acres $=405$ hectares.

1,000 board feet (International 1/4-inch log rule) = 3.48 cubic meters.

Breast height $=1.4$ meters above the ground.

1 cubic foot $=0.0283$ cubic meter.

1 foot $=30.48$ centimeters or 0.3048 meter.

1 inch $=25.4$ millimeters, 2.54 centimeters, or 0.0254 meter.

\section{Definition of Terms}

\section{Land-use classes}

Gross area.-The entire area of land and water as determined by the Bureau of Census, 1970.

Land area.-The area of dry land and land temporarily or partially covered by water such as marshes, swamps, flood plains, streams, sloughs, and estuaries. Canals less than 1/8-mile wide and lakes, reservoirs, and ponds smaller than 40 acres are included as land area. These figures are from the Bureau of Census, 1970.

Forest land.-Land at least 16.7 percent stocked by trees of any size, or formerly having such tree cover, and not currently developed for nonforest use. Includes afforested areas. The minimum forest area classified was 1 acre. Roadside, streamside, and shelterbelt strips of timber must have a crown width of at least 120 feet to qualify as forest land. Unimproved roads and trails, streams, and clearings in forest areas were classed as forest if less than 120 feet wide.

Commercial forest land.-Forest land that is producing or is capable of producing crops of industrial wood and that is not withdrawn from timber utilization by statute or administrative regulation. This includes areas capable of producing in excess of 20 cubic feet per acre of annual growth. This includes both inaccessible and inoperable areas.

Noncommercial forest land.-(a) Unproductive-forest land incapable of yielding crops of industrial wood because of adverse site conditions, (b) productive-reserved-forest land withdrawn from commercial timber use through statute or administrative regulation, or exclusively used for Christmas tree production. 
Nonforest land.-Land that has never supported forests, and land formerly forested where forest use is precluded by development for nonforest uses, such as cropland, improved pasture, residential areas, and city parks. Also includes improved roads and adjoining rights-of-way, powerline clearings, and certain areas of water classified by the Bureau of Census as land. Unimproved roads, streams, canals, and nonforest strips in forest areas must be more than 120 feet wide and clearings in forested areas must be more than $l$ acre in size to qualify as nonforest land.

\section{Ownership classes}

National forest.-Federal land that has been designated by executive order or statute as National Forests or purchase units and other land under the administration of the USDA Forest Service.

Other Federal.-Federal land other than National Forest.

State, county, and municipal.-Land owned by States, counties, or local public agencies, or land leased by them for more than 50 years.

Forest industry.-Land owned by companies or individuals operating primary wood-using plants.

Farmer-owned.-Land owned by operators of farms. A farm must include 10 or more acres from which the sale of agricultural products totals $\$ 50$ or more annually, or if less than 10 acres, the yield must be at least $\$ 250$ annually.

Farmer-owned, leased.-Land owned by operators of farms but leased to other parties.

Miscellaneous private-corporation.-Land owned by a private corporation not in the business of operating primary wood-using plants.

Miscellaneous private-individual._Land owned by a private individual. Miscellaneous private-corporation, leased.-Land owned by private corporations but leased to other parties. Miscellaneous private-individual, leased.-Land owned by private individuals but leased to other parties.

\section{Tree classes}

All live trees.-Growing-stock, rough, and rotten trees 1 inch d.b.h. and larger.

Growing-stock trees.-All live trees of commercial species except rough and rotten trees.

Desirable trees.-Growing-stock trees having no serious quality defects that would limit present or prospective use, possessing relatively high vigor, and containing no pathogens that may kill or seriously deteriorate it before rotation age. These are trees that would be favored by forest managers in silvicultural operations.

Acceptable trees.-Trees meeting the standards for growing stock but not qualifying as desirable trees.

Sawtimber trees.-Growing-stock trees of commercial species containing at least a 12 -foot saw log or two noncontiguous saw logs, each 8 feet or longer. At least 33 percent of the gross volume of the tree must be sound wood. Softwoods must be at least 9 inches d.b.h. and hardwoods at least 11 inches d.b.h.

Poletimber trees.-Growing-stock trees of commercial species at least 5.0 inches d.b.h. but smaller than sawtimber size, and of good form and vigor.

Saplings.-Live trees of commercial species 1 to 5 inches d.b.h. and of good form and vigor.

Seedlings.-Live trees of commercial species less than 1 inch d.b.h. that are expected to survive according to regional standards (examples of seedlings not expected to survive are those that are diseased or heavily damaged by logging, browsing, or fire). Only softwood seedlings more than 6 inches tall and hardwood seedlings more than 1 foot tall are counted.

Rotten trees.-Live trees (any size) of commercial species that do not contain a merchantable 12foot saw log or two noncontiguous 8-foot or longer saw logs, now or prospectively, because of rot (that is, when more than 50 percent of the cull volume of the tree is rotten).

Rough trees.-Live trees that do not contain at least one merchantable 12 -foot saw log or two noncontiguous 8-foot or longer saw logs, now or prospectively, because of roughness and poor form. Includes all live noncommercial species.

Short-log (rough trees).-Sawtimber-sized trees of commercial species that contain at least one merchantable 8- to 11-foot saw log but not a 12 -foot saw log.

\section{Stocking}

The degree of land utilization by trees as measured in terms of basal area and or the number of trees in a stand compared to the basal area and/or number of trees required to fully utilize the growth potential of the land.

A stocking percent of 100 indicates full utilization of the site and is equivalent to 80 square feet of basal 
area per acre in trees 5 inches d.b.h. and larger. In a stand of trees less than 5 inches d.b.h., a stocking percent of 100 would indicate that the present number of trees is sufficient to produce 80 square feet of basal area per acre when the trees reach 5 inches d.b.h.

Stocking of all live trees, growing-stock trees, and desirable trees are recorded separately and stands are grouped into the following stocking classes.

\section{Stocking classes}

Overstocked stands.--Stands in which stocking of trees is 133 percent or more.

Fully-stocked stands.-Stands in which stocking of trees is from 100 to 133 percent.

Medium-stocked stands.-Stands in which stocking of trees is from 60 to 100 percent.

Poorly-stocked stands.-Stands in which stocking of trees is from 16.7 to 60 percent.

Nonstocked areas.-Commercial forest land on which stocking of trees is less than 16.7 percent.

Stand-size classes

Stand.-A growth of trees on a minimum of 1 acre of forest land that is stocked by trees of any size.

Sawtimber stands.-Stands at least 16.7 percent stocked with growing-stock trees, at least half of which are sawtimber or poletimber and sawtimber stocking at least equal to that of poletimber.

Poletimber stands.-Stands at least 16.7 percent stocked with growing-stock trees, at least half of which are sawtimber and/or poletimber trees and poletimber stocking exceeding that of sawtimber.

Sapling-seedling stands.-Stands at least 16.7 percent stocked with growing-stock trees and with saplings and/or seedlings comprising more than half of this stocking.

Nonstocked areas.-Commercial forest land on which stocking of growing-stock trees is less than 16.7 perent.

\section{Other classifications}

Site index.-An expression of forest site quality based on the height of a free-growing dominant or codominant tree of a representative species in the forest type at age 50 .
Site class.-A classification of forest land in terms of inherent capacity to grow crops of industrial wood expressed in cubic-foot growth per acre per year.

Stand-age.-Age of the main stand. Main stand refers to trees of the dominant forest type and standsize class.

Basal area.-The area in square feet of the cross section at breast height of a single tree. When the basal area of all the trees in a stand are summed, the result is usually expressed as square feet of basal area per acre.

\section{Biomass}

The above-ground volume of all live trees (including bark and foliage) reported in green tons. Biomass is made up of 5 components:

Growing-stock bole.-Biomass of a growingstock tree from a 1-foot stump to a variable 4-inch top.

Growing-stock tops and limbs.-Biomass of a growing-stock tree from a 1 -foot stump minus the growing-stock bole.

Cull bole.-Biomass of a cull tree from a 1-foot stump to a variable 4 -inch top.

Cull tops and limbs.-Biomass of a cull tree from a 1-inch stump minus the cull bole.

1- to 5-inch trees.-Biomass of all live trees 1- to 5 -inches in diameter at breast height.

\section{Forest types}

A classification of forest land based on the species forming a plurality of live-tree stocking. Major forest types in Minnesota are:

Jack pine.-Forests in which jack pine comprises a plurality of the stocking. (Common associates include eastern white pine, red pine, aspen, birch, and maple.)

Red pine.-Forests in which red pine comprises a plurality of the stocking. (Common associates include eastern white pine, jack pine, aspen, birch, and maple.)

White pine.-Forests in which eastern white pine comprises a plurality of the stocking. (Common associates include red pine, jack pine, aspen, birch, and maple.)

Balsam fir.-Forests in which balsam fir and white spruce comprise a plurality of stocking with balsam fir the most common. (Common associates 
include white spruce, aspen, maple, birch, northern white-cedar, and tamarack.)

White spruce.-Forests in which white spruce and balsam fir comprise a plurality of the stocking with white spruce the most common. (Common associates include balsam fir, aspen, maple, birch, northern white-cedar, and tamarack.)

Black spruce.-Forests in which swamp conifers comprise a plurality of the stocking with black spruce the most common. (Common associates include tamarack and northern white-cedar.)

Northern white-cedar.-Forests in which swamp conifers comprise a plurality of the stocking with northern white-cedar the most common. (Common associates include tamarack and black spruce.)

Tamarack.-Forests in which swamp conifers comprise a plurality of the stocking with tamarack the most common. (Common associates include black spruce and northern white-cedar.)

Oak.-Forests in which northern red oak, white oak, or bur oak, singly or in combination, comprise a plurality of the stocking. (Common associates include elm, maple, and aspen.)

Elm-ash-cottonwood.-Forests in which lowland elm, ash, cotton wood, and red maple, singly or in combination, comprises a plurality of the stocking. (Common associates include basswood and balsam poplar.)

Maple-basswood.-Forests in which sugar maple, basswood, yellow birch, upland American elm, and red maple, singly or in combination, comprises a plurality of the stocking. (Common associates include white pine and elm.)

Aspen.-Forests in which quaking aspen or bigtooth aspen, singly or in combination, comprises a plurality of the stocking. (Common associates include balsam poplar, balsam fir, and paper birch.)

Paper birch.-Forests in which paper birch comprises a plurality of the stocking. (Common associates include maple, aspen, and balsam fir.)

Balsam poplar._Forests in which balsam poplar comprises a plurality of the stocking. (Common associates include aspen, elm, and ash.)

\section{Timber volume}

Volume of growing stock.-The volume of sound wood in the bole of growing-stock trees 5 inches d.b.h. and over, from a 1 -foot stump to a minimum 4 inch top diameter outside bark, or to the point where the central stem breaks into limbs. Growing-stock volumes are shown in cubic feet. They can be converted to cords by multiplying by 79 (79 cubic feet/ solid wood cord).

Volume of sawtimber.-Net volume of the saw log portion of live sawtimber trees in board feet, International $1 / 4$-inch rule, from stump to a minimum 7 inches top diameter outside bark (d.o.b.) for softwoods and a minimum 9 inches top d.o.b. for hardwoods.

Upper stem portion.-That part of the bole of sawtimber trees above the merchantable sawtimber top to a minimum top diameter of 4 inches outside bark or to the point where the central stem breaks into limbs. Growth and mortality

Net annual growth of growing stock.-The annual change in volume of sound wood in live growing-stock and sawtimber trees and total volume of trees entering these classes through ingrowth, less volume losses resulting from natural causes.

Net annual growth of sawtimber.-The annual change in volume of live sawtimber trees and the total volume of trees reaching sawtimber size, less volume losses resulting from natural causes.

Mortality of growing stock.-The volume of sound wood in growing-stock trees dying annually.

Mortality of sawtimber.-The net board-foot volume of sawtimber trees dying annually.

\section{Timber removals}

Timber removals from growing stock.-The volume of sound wood in growing-stock trees removed annually for forest products (including roundwood products and logging residues) and for other removals. Roundwood products are logs, bolts, or other round sections cut and used from trees. Logging residues are the unused portions of cut trees plus unused trees killed by logging. Other removals are growing-stock trees removed but not utilized for products or trees left standing but "removed" from the commercial forest land classification by land use change- examples are removals from cultural operations such as timber stand improvement work, land clearing, and changes in land use.

Timber removals from sawtimber.-The net board-foot volume of live sawtimber trees removed for 
forest products annually (including roundwood products and logging residues) and for other removals.

Timber products output.-All timber products cut from roundwood, and byproducts of wood manufacturing plants. Roundwood products include logs, bolts, or other round sections cut from growing-stock trees, cull trees, salvable dead trees, trees on nonforest land, noncommercial species, sapling-size trees, and limbwood. Byproducts from primary manufacturing plants include slabs, edgings, trimmings, miscuts, sawdust, shavings, veneer cores and clippings, and screenings of pulpmills that are used as pulpwood chips or other products.

Plant byproducts.-Wood products, such as pulpwood chips, obtained incidental to production of other manufactured products.

Plant residues.-Wood materials from manufacturing plants not utilized for some product.

Table 1.--Area of 1 and by 1 and class, Minnesota, 1962 - / and 1977

(In thousand acres)

\begin{tabular}{|c|c|c|}
\hline Land class & $19621 /$ & 1977 \\
\hline $\begin{array}{l}\text { FOREST: } \\
\text { Commercial forest land: } \\
\text { Jack pine } \\
\text { Red pine } \\
\text { White pine } \\
\text { Balsam fir } \\
\text { White spruce } \\
\text { Black spruce } \\
\text { Northern white-cedar } \\
\text { Tamarack } \\
\text { Oak-hickory } \\
\text { Elm-ash-cottonwood } \\
\text { Maple-basswood } \\
\text { Aspen } \\
\text { Paper birch } \\
\text { Balsam poplar } \\
\text { Nonstocked } \\
\text { Subtotal }\end{array}$ & $\begin{array}{r}872.0 \\
280.6 \\
132.0 \\
907.9 \\
57.3 \\
1,152.3 \\
333.7 \\
470.5 \\
1,022.7 \\
1,286.4 \\
1,004.3 \\
5,399.8 \\
795.1 \\
447.5 \\
1,249.7 \\
15,411.8\end{array}$ & $\begin{array}{r}504.4 \\
246.9 \\
65.6 \\
859.1 \\
79.2 \\
1,041.8 \\
498.6 \\
465.4 \\
893.9 \\
738.1 \\
1,283.9 \\
5,302.3 \\
997.6 \\
548.9 \\
169.4 \\
13,695.1\end{array}$ \\
\hline $\begin{array}{l}\text { Noncommercial forest } 1 \text { and: } \\
\text { Unproductive } \\
\text { Productive-reserved } \\
\text { Subtotal }\end{array}$ & $\begin{array}{r}2,563.1 \\
470.1 \\
3,033.2 \\
\end{array}$ & $\begin{array}{l}1,835.5 \\
1,178.6 \\
3,014.1 \\
\end{array}$ \\
\hline Total & $18,445.0$ & $16,709.2$ \\
\hline NONFOREST LAND: & $32,760.8$ & $34,035.6$ \\
\hline Total & $51,205.8$ & $50,744.8$ \\
\hline
\end{tabular}


Table 2.--Area by land $\mathrm{class}$ and Forest Survey Unit, Minnesota, 1977

(In thousand acres)

\begin{tabular}{|c|c|c|c|c|c|}
\hline Land class & $\begin{array}{c}\text { AlT } \\
\text { units }\end{array}$ & $\begin{array}{l}\text { Aspen- } \\
\text { Birch } \\
\end{array}$ & $\begin{array}{c}\text { Northern } \\
\text { Pine } \\
\end{array}$ & $\begin{array}{l}\text { Central } \\
\text { Hardwood }\end{array}$ & Prairie \\
\hline $\begin{array}{l}\text { FOREST LAND: } \\
\text { Commercial } \\
\text { Productive-reserved } 1 / \\
\text { Unproductive } \\
\text { Total }\end{array}$ & $\begin{array}{r}13,695 \cdot 1 \\
1,178 \cdot 6 \\
1,835 \cdot 5 \\
16,709 \cdot 2 \\
\end{array}$ & $\begin{array}{r}5,451.4 \\
1,050.6 \\
969.8 \\
7,471.8 \\
\end{array}$ & $\begin{array}{r}5,758.4 \\
46.9 \\
706.9 \\
6,512.2 \\
\end{array}$ & $\begin{array}{r}1,951.1 \\
72.4 \\
120.2 \\
2,143.1 \\
\end{array}$ & $\begin{array}{r}534.2 \\
8.7 \\
38.6 \\
581.5 \\
\end{array}$ \\
\hline $\begin{array}{l}\text { NONFOREST LAND: } \\
\text { Nonforest with trees: } \\
\text { Cropland } \\
\text { Improved pasture } \\
\text { Wooded strips } \\
\text { Idle farmland } \\
\text { Windbreaks } \\
\text { Wooded pasture } \\
\text { Subtotal }\end{array}$ & $\begin{array}{r}65.7 \\
90.1 \\
158.9 \\
7.9 \\
151.0 \\
135.0 \\
608.6 \\
\end{array}$ & $\begin{array}{r}4.8 \\
10.6 \\
1.6 \\
13.5 \\
23.3 \\
53.8 \\
\end{array}$ & $\begin{array}{r}20.8 \\
29.4 \\
24.6 \\
1.4 \\
12.3 \\
22.1 \\
110.6 \\
\end{array}$ & $\begin{array}{r}35.4 \\
50.0 \\
99.4 \\
3.9 \\
103.1 \\
80.5 \\
372.3\end{array}$ & $\begin{array}{r}9.5 \\
5.9 \\
24.3 \\
1.0 \\
22.1 \\
9.1 \\
71.9\end{array}$ \\
\hline $\begin{array}{l}\text { Nonforest without trees: } \\
\text { Cropland } \\
\text { Improved pasture } \\
\text { Idle farmland } \\
\text { Marsh } \\
\text { Other farm-farmstead } \\
\text { Urban and other } \\
\text { Noncensus water } \\
\text { Subtotal }\end{array}$ & $\begin{array}{r}26,421.3 \\
897.7 \\
47.1 \\
2,961.6 \\
570.5 \\
2,406.7 \\
122.1 \\
33,427.0 \\
\end{array}$ & $\begin{array}{r}357.1 \\
67.6 \\
15.4 \\
350.6 \\
33.6 \\
283.5 \\
-- \\
1,107.8\end{array}$ & $\begin{array}{r}2,007.2 \\
527.3 \\
22.3 \\
1,449.9 \\
43.3 \\
350.2 \\
32.1 \\
4,432.3\end{array}$ & $\begin{array}{r}7,218.5 \\
159.5 \\
5.8 \\
662.5 \\
219.0 \\
1,085.7 \\
52.2 \\
9,403.2\end{array}$ & $\begin{array}{r}16,838.5 \\
143.3 \\
3.6 \\
498.6 \\
274.6 \\
687.3 \\
37.8 \\
18,483.7\end{array}$ \\
\hline Total & $34,035.6$ & $1,161.6$ & $4,542.9$ & $9,775.5$ & $18,555.6$ \\
\hline Total 1 and area $2 /$ & $50,744.8$ & $8,633.4$ & $11,055.1$ & $11,919.2$ & $19,137.1$ \\
\hline Census water & $3,058.7$ & 715.1 & $1,437.7$ & 581.9 & 324.0 \\
\hline Total gross area & $53,803.5^{4}$ & $9,348.5$ & $12,492.8$ & $12,501.1$ & $19,461.1$ \\
\hline
\end{tabular}

$1 /$ Includes 3,000 acres of productive-deferred forest 1 and, commercial forest 1 and being withheld from harvest while a decision is made whether to $\mathrm{place}$ it in productive-reserved status.

2/U.S. Department of Commerce, Bureau of Census. 1970. Area measurement reports, GE-20, No. $1,22 \mathrm{p}$. 


\begin{tabular}{|c|c|c|c|c|c|}
\hline \multicolumn{6}{|c|}{ ASPEN-BIRCH } \\
\hline \multirow[b]{2}{*}{ County } & \multirow[b]{2}{*}{$\begin{array}{l}\text { Land } 1 / \\
\text { area- }\end{array}$} & \multicolumn{3}{|c|}{ Forest land } & \multirow{2}{*}{$\begin{array}{c}\text { Percent } \\
\text { commercial } \\
\text { forest }\end{array}$} \\
\hline & & $\begin{array}{l}\text { AाT } \\
\text { forest }\end{array}$ & $\begin{array}{l}\text { Non- } \\
\text { commercial }\end{array}$ & Commercial & \\
\hline & -- & \multicolumn{3}{|c|}{-Thousand acres- - - } & \\
\hline Carlton & 551.8 & 336.1 & 23.3 & 312.8 & 57 \\
\hline Cook & 861.4 & 852.9 & 314.1 & 538.8 & 63 \\
\hline Koochiching & $2,001.3$ & $1,794.3$ & 515.4 & $1,278.9$ & 64 \\
\hline Lake & $1,319.8$ & $1,257.1$ & 401.8 & 855.3 & 65 \\
\hline \multirow{2}{*}{$\begin{array}{l}\text { St. Louis } \\
\text { All Counties }\end{array}$} & $3,899.1$ & $3,231.4$ & 765.8 & $2,465.6$ & 63 \\
\hline & $8,633.4$ & $7,471.8$ & $2,020.4$ & $5,451.4$ & 63 \\
\hline \multicolumn{6}{|c|}{ NORTHERN PINE } \\
\hline Aitkin & $1,169.7$ & 762.6 & 90.1 & 672.5 & 58 \\
\hline Becker & 830.0 & 322.8 & 8.9 & 313.9 & 38 \\
\hline Bel trami & $1,604.2$ & $1,044.0$ & 249.4 & 794.6 & 50 \\
\hline Cass & $1,278.8$ & 883.4 & 24.8 & 858.6 & 67 \\
\hline Clearwater & 639.9 & 333.7 & 32.0 & 301.7 & 47 \\
\hline Crow Wing & 636.5 & 380.7 & 8.8 & 371.9 & 58 \\
\hline Hubbard & 596.2 & 403.2 & 5.2 & 398.0 & 67 \\
\hline Itasca & $1,685.3$ & $1,331.6$ & 50.6 & $1,281.0$ & 76 \\
\hline Lake of the Woods & 838.8 & 596.1 & 235.5 & 360.6 & 43 \\
\hline Mahnomen & 360.2 & 107.8 & 1.4 & 106.4 & 30 \\
\hline Roseau & $1,072.8$ & 232.6 & 40.7 & 191.9 & 18 \\
\hline Wadena & 342.7 & 113.7 & 6.4 & 107.3 & 31 \\
\hline All Counties & $11,055.1$ & $6,512.2$ & 753.8 & $5,758.4$ & 52 \\
\hline \multicolumn{6}{|c|}{ CENTRAL HARDWOODS } \\
\hline Anoka & 271.0 & 42.9 & 6.3 & 36.6 & 14 \\
\hline Benton & 257.4 & 28.3 & 2.2 & 26.1 & 10 \\
\hline Carver & 229.9 & 12.3 & 1.9 & 10.4 & 5 \\
\hline Chisago & 268.1 & 55.7 & 5.5 & 50.2 & 19 \\
\hline Dakota & 368.4 & 19.5 & 3.0 & 16.5 & 5 \\
\hline Douglas & 413.9 & 20.4 & 1.9 & 18.5 & 5 \\
\hline Fillmore & 549.8 & 72.9 & 7.6 & 65.3 & 12 \\
\hline Goodhue & 482.1 & 60.7 & 4.3 & 56.4 & 12 \\
\hline Hennepin & 363.1 & 11.4 & 3.6 & 7.8 & 2 \\
\hline Houston & 361.4 & 119.6 & 8.1 & 111.5 & 31 \\
\hline Isanti & 280.6 & 54.1 & 7.2 & 46.9 & 17 \\
\hline Kanabec & 335.1 & 134.5 & 5.5 & 129.0 & 39 \\
\hline LeSueur & 281.6 & 11.4 & 1.4 & 10.0 & 4 \\
\hline Mille Lacs & 365.6 & 136.2 & 13.4 & 122.8 & 34 \\
\hline Morrison & 721.4 & 161.3 & 12.6 & 148.7 & 21 \\
\hline 01msted & 419.6 & 35.3 & 3.3 & 32.0 & 8 \\
\hline Otter Tail & $1,256.2$ & 200.5 & 14.2 & 186.3 & 15 \\
\hline Fine & 904.7 & 477.6 & 52.0 & 425.6 & 47 \\
\hline Ramsey & 99.0 & 0.1 & 0.1 & -- & 0 \\
\hline Rice & 317.6 & 14.1 & 2.0 & 12.1 & 4 \\
\hline Scott & 225.7 & 16.3 & 2.7 & 13.6 & 6 \\
\hline Sherburne & 275.8 & 62.3 & 5.4 & 56.9 & 21 \\
\hline Stearns & 858.9 & 60.3 & 4.3 & 56.0 & 7 \\
\hline Todd & 602.6 & 111.1 & 6.6 & 104.5 & 17 \\
\hline Wabasha & 334 & 62.1 & 4.7 & 57.4 & 17 \\
\hline Washington & 246 & 12.1 & 1.9 & 10.2 & 4 \\
\hline Winona & 397 & 114.9 & 8.6 & 106.3 & 27 \\
\hline Wright & 431.6 & 35.8 & 2.3 & 33.5 & 8 \\
\hline All Counties & $11,919.2$ & $2,143.7$ & 192.6 & $1,951.1$ & 16 \\
\hline
\end{tabular}




\begin{tabular}{|c|c|c|c|c|c|}
\hline \multirow[b]{2}{*}{ County } & \multirow[b]{2}{*}{$\begin{array}{l}\text { Land } 1 / \\
\text { area- }\end{array}$} & \multicolumn{3}{|c|}{ Forest Tand } & \multirow{2}{*}{$\begin{array}{c}\text { Percent } \\
\text { commercial } \\
\text { forest }\end{array}$} \\
\hline & & $\begin{array}{l}\text { AाI } \\
\text { forest }\end{array}$ & $\begin{array}{l}\text { Non- } \\
\text { commercial }\end{array}$ & Commercial & \\
\hline & -- & - - - Tho & and acres- - & $---\cdot-$ & \\
\hline Big Stone & 313.8 & 2.7 & 0.3 & 2.4 & 1 \\
\hline Blue Earth & 471.3 & 23.6 & 2.2 & 21.4 & 5 \\
\hline Brown & 390.3 & 10.7 & 1.6 & 9.1 & 2 \\
\hline Chippewa & 372.2 & 4.0 & 0.1 & 3.9 & 1 \\
\hline Clay & 669.1 & 11.2 & 0.9 & 10.3 & 2 \\
\hline Cottonwood & 407.0 & 2.6 & 0.1 & 2.5 & 1 \\
\hline Dodge & 278.4 & 7.4 & 1.0 & 6.4 & 2 \\
\hline Faribault & 455.1 & 6.5 & 0.3 & 6.2 & 1 \\
\hline Freeborn & 448.4 & 5.7 & 0.9 & 4.8 & 1 \\
\hline Grant & 349.6 & 3.0 & 0.1 & 2.9 & 1 \\
\hline Jackson & 445.5 & 2.3 & 0.2 & 2.1 & 1 \\
\hline Kandiyohi & 501.2 & 12.4 & 2.5 & 9.9 & 2 \\
\hline Kittson & 719.0 & 71.9 & 4.4 & 67.5 & 9 \\
\hline Lac qui Parle & 491.6 & 5.2 & 0.6 & 4.6 & 1 \\
\hline Lincoln & 339.8 & 2.2 & 0.2 & 2.0 & 1 \\
\hline Lyon & 453.5 & 5.3 & 0.9 & 4.4 & 1 \\
\hline McLeod & 312.3 & 6.1 & 0.3 & 5.8 & 2 \\
\hline Marshall & $1,145.1$ & 142.7 & 14.2 & 128.5 & 11 \\
\hline Martin & 450.1 & 3.8 & 0.2 & 3.6 & 1 \\
\hline Meeker & 396.3 & 12.0 & 1.2 & 10.8 & 3 \\
\hline Mower & 449.9 & 6.1 & 0.9 & 5.2 & 1 \\
\hline Murray & 449.9 & 1.4 & 0.3 & 1.1 & 0 \\
\hline Nicollet & 276.7 & 13.7 & 1.4 & 12.3 & 4 \\
\hline Nobles & 455.5 & 0.7 & -- & 0.7 & 0 \\
\hline Norman & 566.4 & 23.1 & 0.9 & 22.2 & 4 \\
\hline Pennington & 398.0 & 32.0 & 1.3 & 30.7 & 8 \\
\hline Pipestone & 297.0 & 0.4 & $\ldots$ & 0.4 & 0 \\
\hline Polk & $1,288.1$ & 71.3 & 3.3 & 68.0 & 5 \\
\hline Pope & 428.3 & 7.8 & 0.9 & 6.9 & 2 \\
\hline Red Lake & 276.5 & 28.9 & 1.7 & 27.2 & 10 \\
\hline Redwood & 559.4 & 6.7 & 0.5 & 6.2 & 1 \\
\hline Renville & 626.3 & 8.0 & 0.8 & 7.2 & 1 \\
\hline Rock & 310.4 & 0.5 & -- & 0.5 & 0 \\
\hline Sibley & 373.2 & 11.9 & 1.0 & 10.9 & 3 \\
\hline Steele & 272.3 & 5.6 & 0.6 & 5.0 & 2 \\
\hline Stevens & 357.2 & 1.0 & -- & 1.0 & 0 \\
\hline Swift & 473.2 & 5.0 & 0.3 & 4.7 & 1 \\
\hline Traverse & 363.7 & 0.9 & -- & 0.9 & 0 \\
\hline Waseca & 265.3 & 5.0 & 0.4 & 4.6 & 2 \\
\hline Watonwan & 276.9 & 1.1 & -- & 1.1 & 0 \\
\hline Wilkin & 481.1 & 0.5 & -- & 0.5 & 0 \\
\hline Yellow Medicine & 482.2 & 8.6 & 0.8 & 7.8 & 2 \\
\hline All Counties & $19,137.1$ & 581.5 & 47.3 & 534.2 & 3 \\
\hline All units & $50,744.8$ & $16,709.2$ & $3,014.1$ & $13,695.1$ & 27 \\
\hline
\end{tabular}

$1 /$ U.S. Department of Commerce, Bureau of Census. 1970. Area measurement reports, GE-20, No. 1, 22 p. 
Table 4.--Area of commercial forest land by ownership

class and Forest Survey Unit, Minnesota, 1977

(In thousand acres)

\begin{tabular}{|c|c|c|c|c|c|}
\hline Ownership class & $\begin{array}{c}\text { AII } \\
\text { units }\end{array}$ & $\begin{array}{l}\text { Aspen- } \\
\text { Birch }\end{array}$ & $\begin{array}{l}\text { Northern } \\
\text { Pine }\end{array}$ & $\begin{array}{l}\text { Central } \\
\text { Hardwood }\end{array}$ & Prairie \\
\hline $\begin{array}{l}\text { National Forest } \\
\text { Bureau of Land Mgmt. } \\
\text { Indian } \\
\text { Miscell aneous federal } \\
\text { State } \\
\text { County and municipal } \\
\text { Forest industry } \\
\text { Farmer } \\
\text { Farmer owned-leased } \\
\text { Misc. private-corp. } \\
\text { Misc. private-indiv. } \\
\text { Misc. priv.-corp., leased } \\
\text { Misc. priv.-indiv., leased }\end{array}$ & $\begin{array}{r}1,715.1 \\
43.9 \\
466.8 \\
110.5 \\
2,650.5 \\
2,341.6 \\
772.0 \\
3,403.7 \\
-- \\
466.7 \\
1,712.0 \\
5.7 \\
6.6 \\
\end{array}$ & $\begin{array}{r}1,152.8 \\
8.1 \\
97.5 \\
20.7 \\
1,132.1 \\
1,185.9 \\
534.7 \\
544.2 \\
-- \\
246.8 \\
523.6 \\
4.0 \\
1.0 \\
\end{array}$ & $\begin{array}{r}562.3 \\
35.8 \\
364.9 \\
60.5 \\
1,228.7 \\
1,096.7 \\
233.8 \\
1,322.1 \\
.-- \\
154.3 \\
696.6 \\
--- \\
2.7\end{array}$ & $\begin{array}{r}-- \\
-- \\
4.4 \\
20.3 \\
244.7 \\
59.0 \\
0.5 \\
1,141.4 \\
-\overline{-} \\
61.1 \\
418.1 \\
-\overline{1.0} \\
1.6\end{array}$ & $\begin{array}{r}-- \\
-- \\
-- \\
9.0 \\
45.0 \\
-- \\
3.0 \\
396.0 \\
-- \\
4.5 \\
73.7 \\
1.7 \\
1.3 \\
\end{array}$ \\
\hline All owners & $13,695.1$ & $5,451.4$ & $5,758.4$ & $1,951.1$ & 534.2 \\
\hline
\end{tabular}


Table 5.--Area of commercial forest land by ownership class and forest type, Minnesota, 1977

(In thousand acres)

\begin{tabular}{|c|c|c|c|c|c|c|c|c|}
\hline \multirow[b]{2}{*}{ Ownership class } & \multirow[b]{2}{*}{$\begin{array}{c}\text { All } \\
\text { types }\end{array}$} & \multicolumn{7}{|c|}{ Forest type } \\
\hline & & $\begin{array}{l}\text { Jack } \\
\text { pine }\end{array}$ & $\begin{array}{l}\text { Red } \\
\text { pine }\end{array}$ & $\begin{array}{l}\text { White } \\
\text { pine }\end{array}$ & $\begin{array}{c}\text { Bal sam } \\
\text { fir }\end{array}$ & $\begin{array}{l}\text { White } \\
\text { spruce }\end{array}$ & $\begin{array}{l}\text { Black } \\
\text { spruce }\end{array}$ & $\begin{array}{l}\text { Northern } \\
\text { white- } \\
\text { cedar }\end{array}$ \\
\hline National Forest & $1,715.1$ & 116.9 & 130.7 & 19.4 & 185.9 & 26.3 & 184.4 & 61.5 \\
\hline Bureau of Land Mgmt. & 43.9 & 6.7 & 1.4 & 1.4 & -- & 1.4 & 6.9 & 6.2 \\
\hline Indian & 466.8 & 5.2 & 8.8 & 7.5 & 23.0 & 6.5 & 51.4 & 48.7 \\
\hline Miscellaneous federal & 110.5 & 5.4 & 1.3 & -- & 6.1 & -- & 4.2 & 1.6 \\
\hline State & $2,650.5$ & 93.8 & 23.7 & 2.6 & 198.7 & 22.3 & 421.2 & 202.0 \\
\hline County and municipal & $2,341.6$ & 82.4 & 24.5 & 4.4 & 196.4 & 4.1 & 160.6 & 68.7 \\
\hline Forest industry & 772.0 & 52.4 & 13.5 & 5.1 & 80.6 & 2.7 & 66.8 & 49.9 \\
\hline Farmer & $3,403.7$ & 80.9 & 21.5 & 16.7 & 75.5 & 7.8 & 69.9 & 25.4 \\
\hline Farmer owned-leased & -- & -- & -- & -- & -- & -- & - & - \\
\hline Misc. private-corp. & 466.7 & 12.4 & 3.6 & 1.0 & 32.4 & -- & 27.2 & 6.6 \\
\hline Misc. private-indiv. & $1,712.0$ & 48.3 & 17.9 & 7.5 & 60.5 & 8.1 & 49.2 & 28.0 \\
\hline Misc.priv.-corp., leased & 5.7 & - & -- & - & -- & -- & - & -- \\
\hline Misc. priv.-indiv., leased & 6.6 & -- & -- & -- & -- & -- & -- & -- \\
\hline All owners & $13,695.1$ & 504.4 & 246.9 & 65.6 & 859.1 & 79.2 & $1,041.8$ & 498.6 \\
\hline
\end{tabular}

(Table 5 continued)

\begin{tabular}{|c|c|c|c|c|c|c|c|c|}
\hline \multirow[b]{2}{*}{ Ownership class } & \multicolumn{8}{|c|}{ Forest type } \\
\hline & Tamarack & $\begin{array}{c}\text { Oak- } \\
\text { hickory }\end{array}$ & $\begin{array}{l}\text { ETm } \\
\text { ash-cotton- } \\
\text { wood }\end{array}$ & $\begin{array}{c}\text { Maple- } \\
\text { basswood }\end{array}$ & Aspen & $\begin{array}{l}\text { Paper } \\
\text { birch }\end{array}$ & $\begin{array}{l}\text { Bal sam } \\
\text { poplar }\end{array}$ & $\begin{array}{c}\text { Non- } \\
\text { stocked }\end{array}$ \\
\hline National Forest & 7.2 & 11.1 & 44.6 & 98.7 & 598.5 & 196.5 & -- & 33.4 \\
\hline Bureau of Land Mgmt. & - & - & 1.6 & - & 13.9 & - & 2.8 & 1.6 \\
\hline Indian & 25.5 & 8.6 & 19.0 & 24.9 & 187.2 & 26.9 & 20.3 & 3.3 \\
\hline Miscellaneous federal & 5.6 & 6.5 & 14.5 & 11.7 & 33.1 & 8.7 & 8.7 & 3.1 \\
\hline State & 223.7 & 65.8 & 123.3 & 117.2 & 846.6 & 129.9 & 140.4 & 39.3 \\
\hline County and municipal & 73.1 & 39.0 & 122.8 & 163.0 & $1,057.9$ & 223.9 & 97.4 & 23.4 \\
\hline Forest industry & 10.0 & 10.3 & 38.5 & 40.2 & 315.8 & 49.0 & 29.0 & 8.2 \\
\hline Farmer & 76.0 & 581.2 & 234.8 & 586.1 & $1,278.8$ & 161.2 & 152.3 & 35.6 \\
\hline Farmer owned-leased & -- & -- & -- & -- & -- & -- & -- & -- \\
\hline Misc. private-corp. & 16.6 & 29.9 & 19.7 & 33.5 & 204.4 & 54.1 & 19.8 & 5.5 \\
\hline Misc. private-indiv. & 27.7 & 140.2 & 119.3 & 205.7 & 758.0 & 147.4 & 78.2 & 16.0 \\
\hline Misc. priv.-corp., leased & -- & -- & -- & -- & 5.7 & -- & -- & -- \\
\hline Misc. priv.-indiv., leased & -- & 1.3 & -- & 2.9 & 2.4 & -- & -- & -- \\
\hline All owners & 465.4 & 893.9 & 738.1 &, 283.9 & $5,302.3$ & 997.6 & 548.9 & 169.4 \\
\hline
\end{tabular}


Table 6.--Area of commercial forest land by ownership

class and site class, Minnesota, 1977

(In thousand acres)

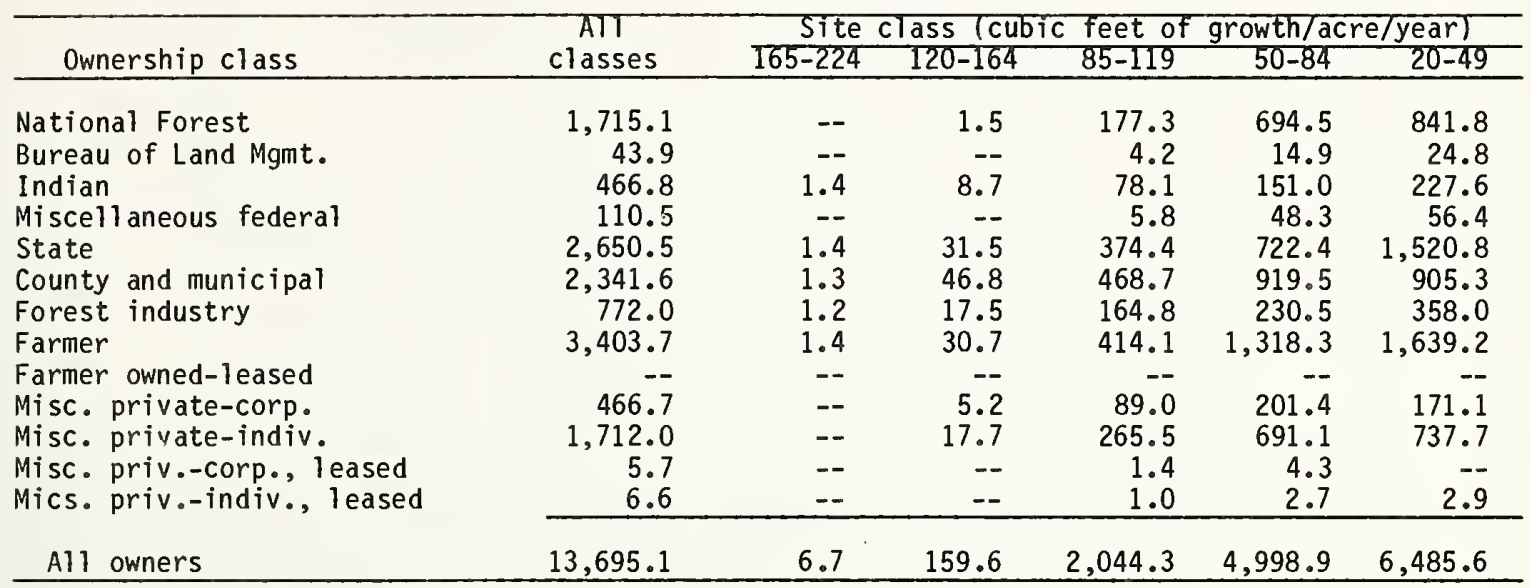




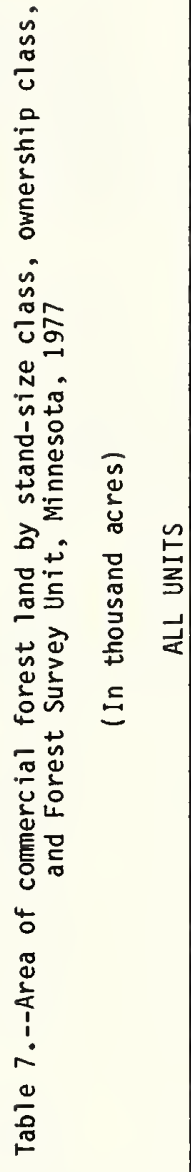

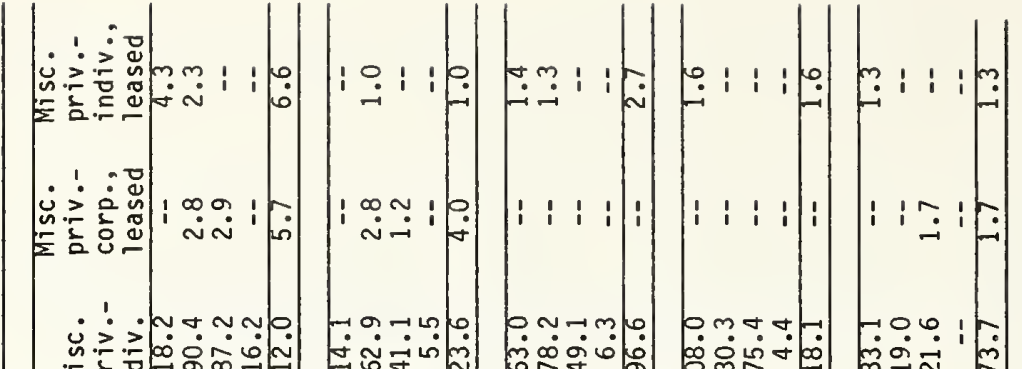

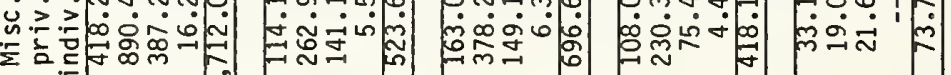
i.j.

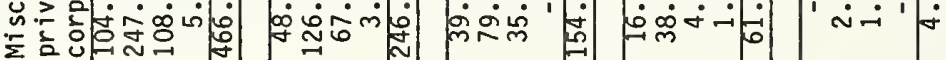
至

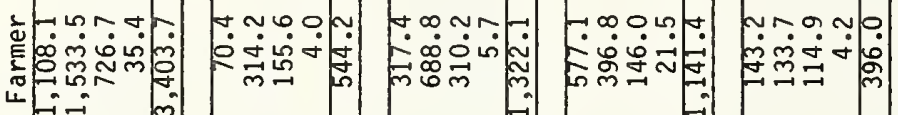

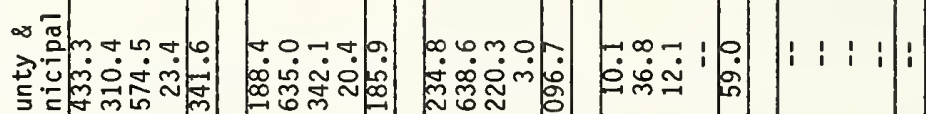
ठํㅣㄹ

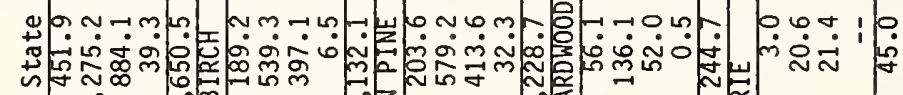

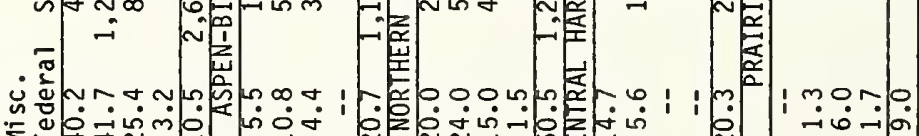

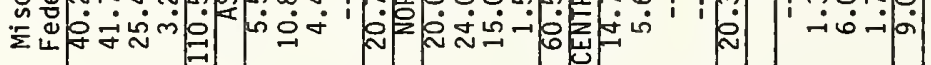

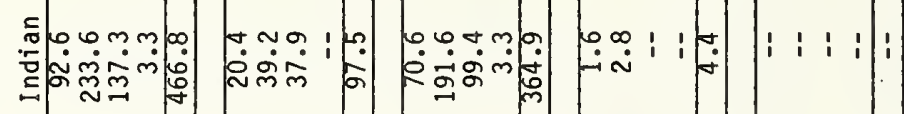
䓃蓄 空

- $0 \rightarrow-7.7$ un a n

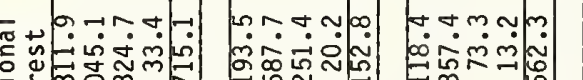
乎 ₹

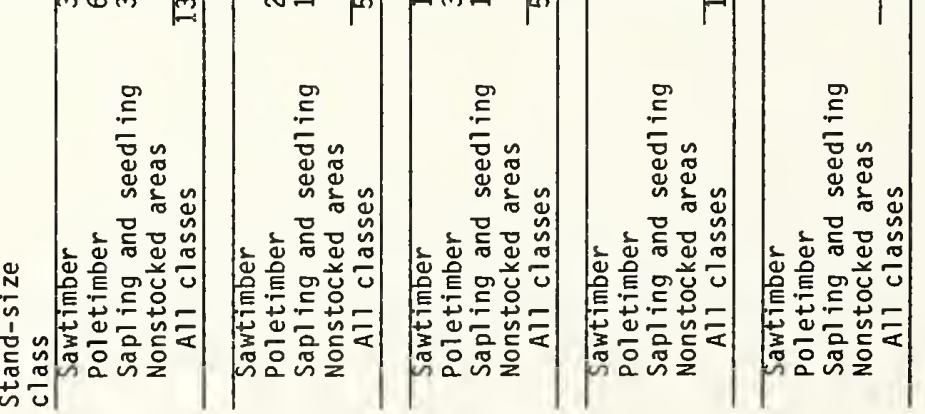


Table 8.--Area of commercial forest land by ownership class and area-condition class, Minnesota, 1977

(In thousand acres)

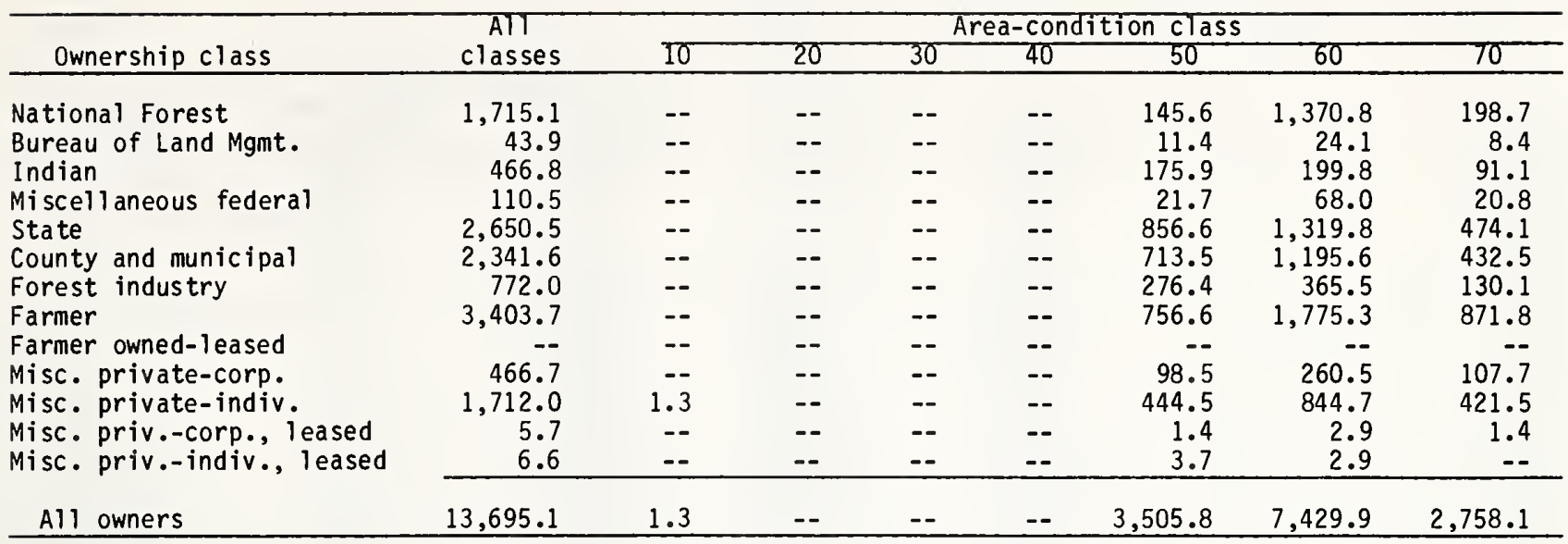

Table 9.--Area of commercial forest land by ownership class and stand-volume class, Minnesota, 1977

(In thousand acres)

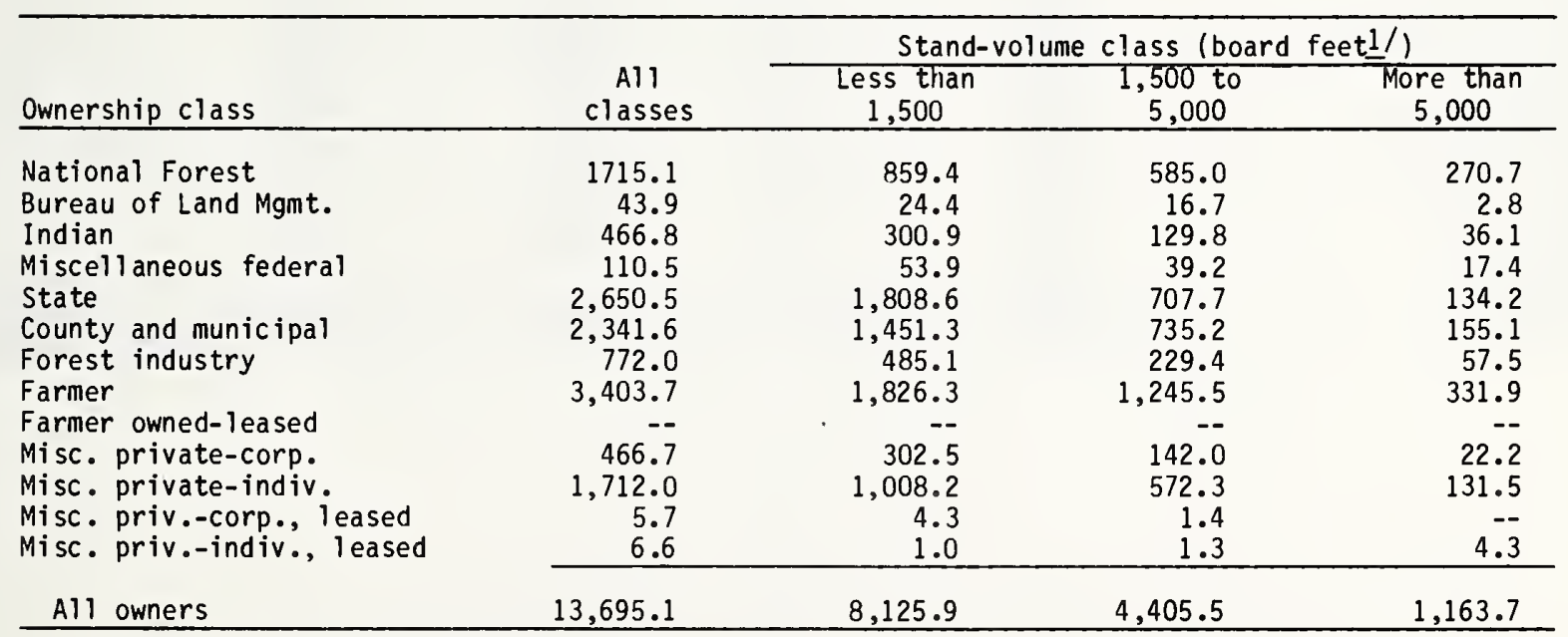

1/International $1 / 4$-inch rule. 
Table 10.--Area of commercial forest land by forest type, stand-size class, and Forest Survey Unit, Minnesota, 1977

(In thousand acres)

ALL UNITS

\begin{tabular}{|c|c|c|c|c|c|}
\hline \multirow[b]{2}{*}{$\begin{array}{l}\text { Forest } \\
\text { type }\end{array}$} & \multirow[b]{2}{*}{$\begin{array}{l}\text { Al l } \\
\text { stands }\end{array}$} & \multicolumn{4}{|c|}{ Stand-size class } \\
\hline & & $\begin{array}{l}\text { Sawtimber } \\
\text { stands }\end{array}$ & $\begin{array}{l}\text { Poletimber } \\
\text { stands }\end{array}$ & $\begin{array}{l}\text { Sapling and } \\
\text { seedling stands }\end{array}$ & $\begin{array}{l}\text { Nonstocked } \\
\text { areas }\end{array}$ \\
\hline Jack pine & 504.4 & 177.3 & 247.2 & 79.9 & -- \\
\hline Red pine & 246.9 & 145.1 & 41.8 & 60.0 & -- \\
\hline White pine & 65.6 & 58.4 & 3.9 & 3.3 & -- \\
\hline Balsam fir & 859.1 & 140.0 & 490.5 & 228.6 & -- \\
\hline White spruce & 79.2 & 21.7 & 23.5 & 34.0 & -- \\
\hline Black spruce & $1,041.8$ & 31.1 & 390.3 & 620.4 & -- \\
\hline Northern white-cedar & 498.6 & 145.5 & 275.5 & 77.6 & -- \\
\hline Tamarack & 465.4 & 43.5 & 200.3 & 221.6 & -- \\
\hline Oak-hickory & 893.9 & 452.7 & 360.9 & 80.3 & -- \\
\hline Elm-ash-cottonwood & 738.1 & 194.8 & 388.8 & 154.5 & -- \\
\hline Maple-basswood & $1,283.9$ & 675.6 & 493.5 & 114.8 & -- \\
\hline Aspen & $5,302.3$ & 791.4 & $3,032.3$ & $1,478.6$ & -- \\
\hline Paper birch & 997.6 & 142.0 & 758.6 & 97.0 & -- \\
\hline Bal sam poplar & 548.9 & 115.7 & 249.0 & 184.2 & -- \\
\hline Nonstocked & 169.4 & -- & -- & $\ldots$ & 169.4 \\
\hline Al1 types & $13,695.1$ & $3,134.8$ & $6,956.1$ & $3,434.8$ & 169.4 \\
\hline \multicolumn{6}{|c|}{ ASPEN-BIRCH } \\
\hline Jack pine & 165.7 & 68.3 & 66.3 & 31.1 & -- \\
\hline Red pine & 114.4 & 49.2 & 19.9 & 45.3 & -- \\
\hline White pine & 33.8 & 32.4 & -- & 1.4 & -- \\
\hline Bal sam fir & 626.0 & 106.1 & 362.9 & 157.0 & -- \\
\hline White spruce & 60.1 & 13.0 & 17.1 & 30.0 & -- \\
\hline Black spruce & 745.7 & 24.0 & 304.4 & 417.3 & -- \\
\hline Northern white-cedar & 317.8 & 110.2 & 153.0 & 54.6 & -- \\
\hline Tamarack & 157.6 & 9.8 & 53.6 & 94.2 & -- \\
\hline Oak-hickory & 5.4 & -- & 5.4 & -- & -- \\
\hline Elm-ash-cottonwood & 243.7 & 45.0 & 138.8 & 59.9 & -- \\
\hline Maple-bas swood & 214.5 & 106.0 & 79.8 & 28.7 & -- \\
\hline Aspen & $1,947.4$ & 260.5 & $1,120.5$ & 566.4 & -- \\
\hline Paper birch & 546.3 & 69.4 & 419.2 & 57.7 & -- \\
\hline Bal sam poplar & 208.0 & 49.0 & 106.2 & 52.8 & -- \\
\hline Nonstocked & 65.0 & -- & - & -- & 65.0 \\
\hline All types & $5,451.4$ & 942.9 & $2,847.1$ & $1,596.4$ & 65.0 \\
\hline \multicolumn{6}{|c|}{ NORTHERN PINE } \\
\hline Jack pine & 327.4 & 105.9 & 174.6 & 46.9 & -- \\
\hline Red pine & 121.9 & 94.3 & 15.6 & 12.0 & -- \\
\hline White pine & 24.8 & 20.9 & 3.9 & - & -- \\
\hline Balsam fir & 225.6 & 31.6 & 124.4 & 69.6 & -- \\
\hline White spruce & 19.1 & 8.7 & 6.4 & 4.0 & -- \\
\hline Black spruce & 278.2 & 7.1 & 80.8 & 190.3 & -- \\
\hline Northern white-cedar & 180.8 & 35.3 & 122.5 & 23.0 & -- \\
\hline Tamarack & 276.0 & 26.1 & 132.2 & 117.7 & -- \\
\hline Oak-hickory & 247.4 & 65.5 & 143.5 & 38.4 & -- \\
\hline Elm-ash-cottonwood & 309.8 & 76.4 & 169.5 & 63.9 & -- \\
\hline Maple-basswood & 466.4 & 179.0 & 252.9 & 34.5 & -- \\
\hline Aspen & $2,541.7$ & 452.4 & $1,451.4$ & 637.9 & -- \\
\hline Paper birch & 371.2 & 55.9 & 288.3 & 27.0 & -- \\
\hline Bal sam poplar & 297.5 & 66.7 & 116.5 & 114.3 & -- \\
\hline Nonstocked & 70.6 & -- & -- & -- & 70.6 \\
\hline All types & $5,758.4$ & $1,225.8$ & $3,082.5$ & $1,379.5$ & 70.6 \\
\hline
\end{tabular}




\begin{tabular}{|c|c|c|c|c|c|}
\hline \multirow{2}{*}{$\begin{array}{l}\text { Forest } \\
\text { type }\end{array}$} & \multirow{2}{*}{$\begin{array}{c}\text { A11 } \\
\text { stands }\end{array}$} & \multicolumn{4}{|c|}{ Stand-size class } \\
\hline & & $\begin{array}{l}\text { Sawt imber } \\
\text { stands }\end{array}$ & $\begin{array}{l}\text { Poletimber } \\
\text { stands }\end{array}$ & Sapling and & $\begin{array}{c}\text { Nonstocked } \\
\text { areas }\end{array}$ \\
\hline Jack pine & $\frac{\operatorname{sen} 05}{11.3}$ & $\frac{3.1}{3.1}$ & $\frac{62 a n d s}{6.3}$ & 1.9 & artas \\
\hline Red pine & 10.6 & 1.6 & 6.3 & 2.7 & -- \\
\hline White pine & 7.0 & 5.1 & -- & 1.9 & -- \\
\hline Balsam fir & 7.5 & 2.3 & 3.2 & 2.0 & -- \\
\hline White spruce & -- & -- & -- & -- & -- \\
\hline Black spruce & 17.9 & -- & 5.1 & 12.8 & -- \\
\hline Northern white-cedar & -- & -- & -- & -- & -- \\
\hline Tamarack & 30.8 & 7.6 & 14.5 & 8.7 & -- \\
\hline 0ak-hickory & 571.8 & 343.2 & 194.0 & 34.6 & -- \\
\hline Elm-ash-cottonwood & 129.5 & 39.1 & 62.7 & 27.7 & -- \\
\hline Maple-basswood & 485.8 & 296.7 & 147.4 & 41.7 & -- \\
\hline As pen & 560.2 & 70.1 & 349.1 & 141.0 & -- \\
\hline Paper birch & 80.1 & 16.7 & 51.1 & 12.3 & -- \\
\hline Balsam poplar & 10.7 & -- & 8.1 & 2.6 & -- \\
\hline Nonstocked & 27.9 & -- & - & -- & 27.9 \\
\hline All types & $1,951.1$ & 785.5 & 847.8 & 289.9 & 27.9 \\
\hline & & PRAIRIE & & & \\
\hline Jack pine & -- & -- & -- & -- & -- \\
\hline Red pine & -- & -- & -- & -- & -- \\
\hline White pine & -- & -- & -- & -- & -- \\
\hline Balsam fir & -- & -- & -- & -- & -- \\
\hline White spruce & -- & -- & -- & -- & -- \\
\hline Black spruce & -- & -- & -- & -- & -- \\
\hline Northern white-cedar & -- & -- & -- & -- & -- \\
\hline Tamarack & 1.0 & -- & -- & 1.0 & -- \\
\hline 0ak-hickory & 69.3 & 44.0 & 18.0 & 7.3 & -- \\
\hline Elm-ash-cottonwood & 55.1 & 34.3 & 17.8 & 3.0 & -- \\
\hline Maple-basswood & 117.2 & 93.9 & 13.4 & 9.9 & -- \\
\hline Aspen & 253.0 & 8.4 & 111.3 & 133.3 & -- \\
\hline Paper birch & -- & -- & & -- & -- \\
\hline Balsam poplar & 32.7 & -- & 18.2 & 14.5 & -- \\
\hline Nonstocked & 5.9 & - & -- & $\ldots$ & 5.9 \\
\hline All types & 534.2 & 180.6 & 178.7 & 169.0 & 5.9 \\
\hline
\end{tabular}




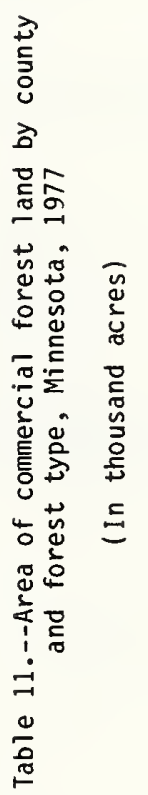

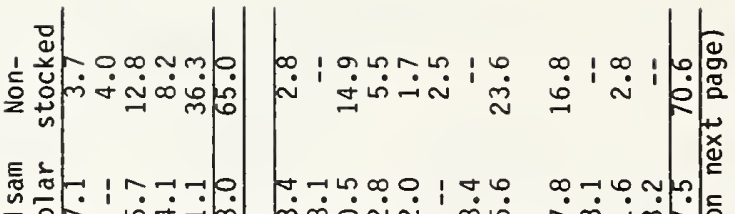

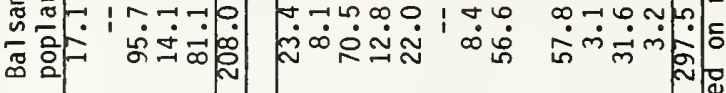

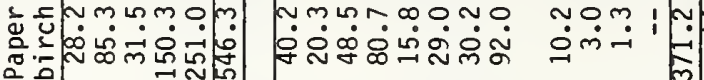

|ris

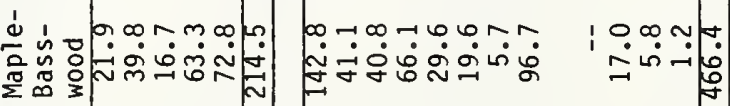

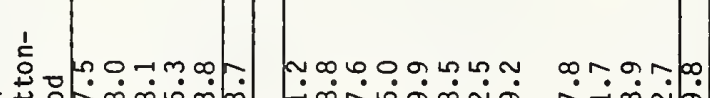

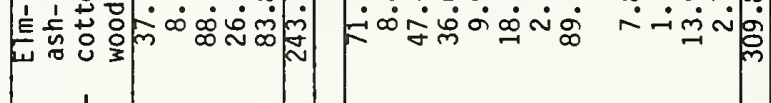

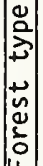

美总

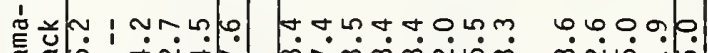

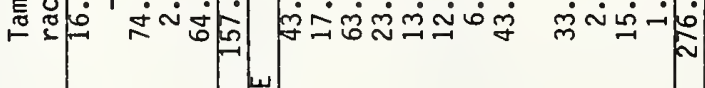

E

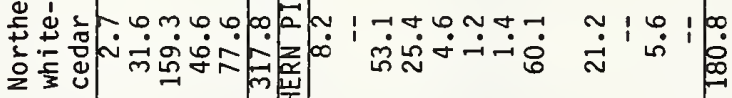

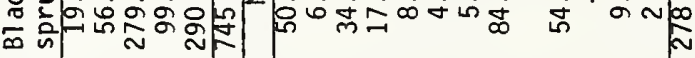

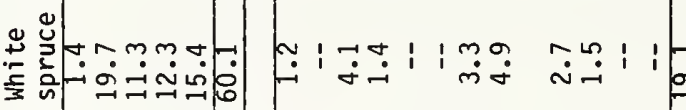

焉

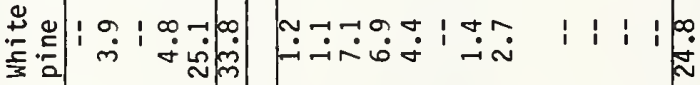

送范

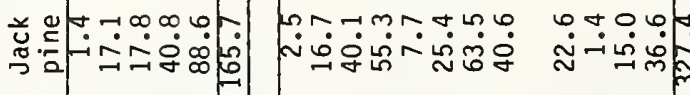

-

ก.

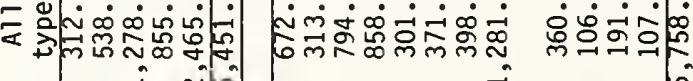

$\rightarrow$ if $\rightarrow$ in

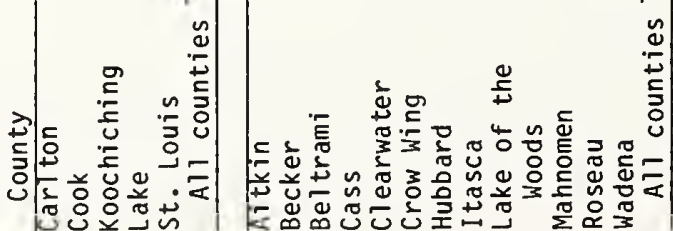




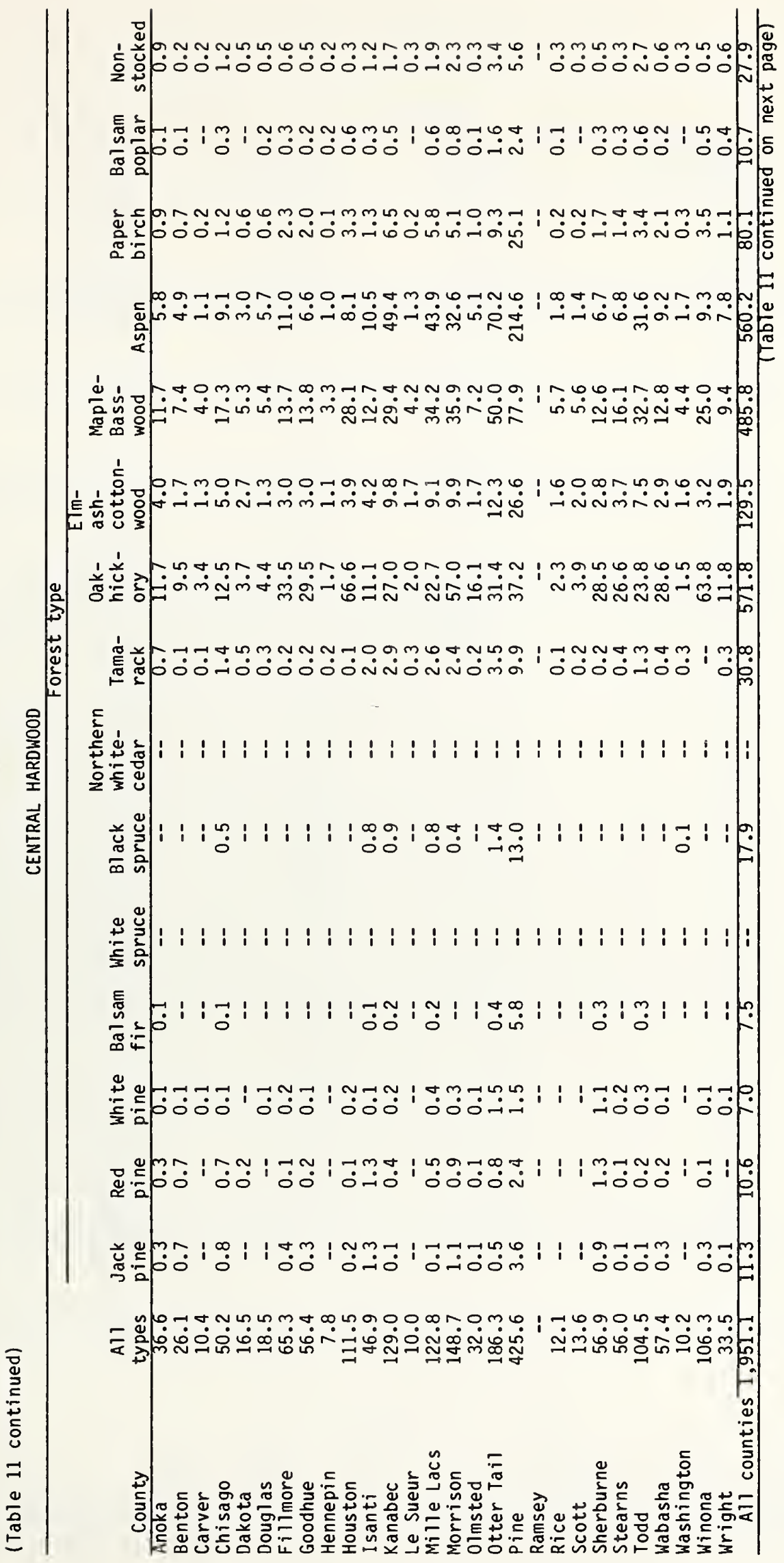




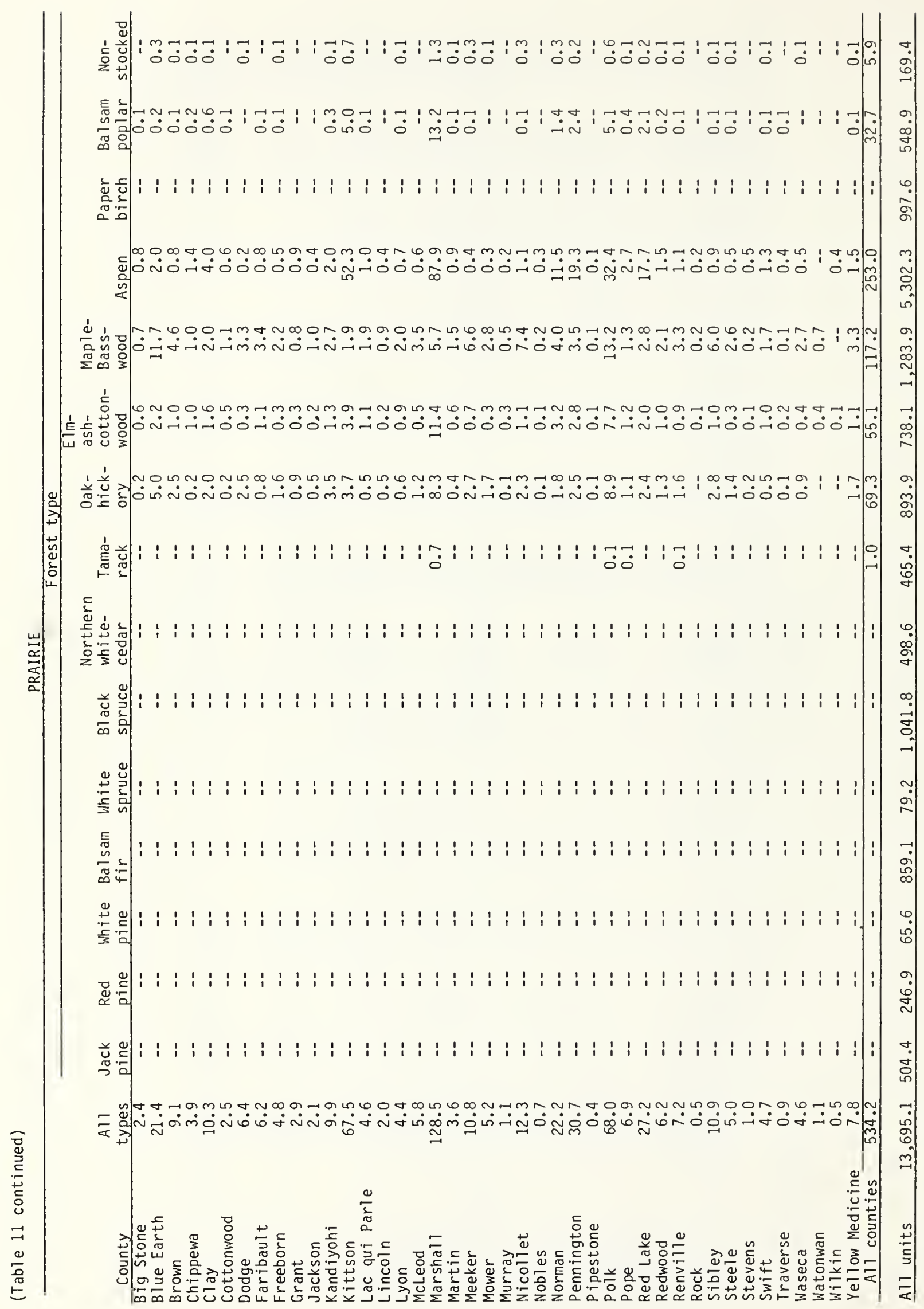


Table 12.--Area of commercial forest land by county and stand-size class, Minnesota, 1977

(In thousand acres)

ASPEN-BIRCH

\begin{tabular}{|c|c|c|c|c|c|}
\hline \multirow[b]{2}{*}{ County } & \multirow[b]{2}{*}{$\begin{array}{l}\text { All } \\
\text { stands }\end{array}$} & \multicolumn{4}{|c|}{ Stand-size cTass } \\
\hline & & $\begin{array}{l}\text { Sawtimber } \\
\text { stands }\end{array}$ & $\begin{array}{l}\text { Poletimber } \\
\text { stands }\end{array}$ & $\begin{array}{c}\text { Sapling and } \\
\text { seedling stands }\end{array}$ & $\begin{array}{c}\text { Nonstocked } \\
\text { areas }\end{array}$ \\
\hline carten & 312.8 & 38.4 & 162.2 & 108.5 & 3.7 \\
\hline Cook & 538.8 & 142.5 & 295.9 & 96.4 & 4.0 \\
\hline Koochiching & $1,278.9$ & 193.7 & 589.2 & 483.2 & 12.8 \\
\hline Lake & 855.3 & 194.8 & 429.9 & 222.4 & 8.2 \\
\hline St. Louis & $2,465.6$ & 373.5 & $1,369.9$ & 685.9 & 36.3 \\
\hline All counties & $5,451.4$ & 942.9 & $2,847.1$ & $1,596.4$ & 65.0 \\
\hline \multicolumn{6}{|c|}{ NORTHERN PINE } \\
\hline Aitkin & 672.5 & 142.0 & 331.1 & 196.6 & 2.8 \\
\hline Becker & 313.9 & 81.1 & 178.9 & 53.9 & - \\
\hline Bel trami & 794.6 & 154.8 & 428.6 & 196.3 & 14.9 \\
\hline Cass & 858.6 & 189.0 & 515.3 & 148.8 & 5.5 \\
\hline Clearwater & 301.7 & 77.5 & 150.4 & 72.1 & 1.7 \\
\hline Crow Wing & 371.9 & 81.7 & 223.4 & 64.3 & 2.5 \\
\hline Hubbard & 398.0 & 71.5 & 242.8 & 83.7 & -- \\
\hline Itasca & $1,281.0$ & 308.5 & 677.2 & 271.7 & 23.6 \\
\hline Lake of the Woods & 360.6 & 49.6 & 144.9 & 149.3 & 16.8 \\
\hline Mahnomen & 106.4 & 22.4 & 56.5 & 27.5 & -- \\
\hline Roseau & 191.9 & 29.7 & 72.3 & 87.1 & 2.8 \\
\hline Wadena & 107.3 & 18.0 & 61.1 & 28.2 & -- \\
\hline All counties & $5,758.4$ & $1,225.8$ & $3,082.5$ & $1,379.5$ & 70.6 \\
\hline \multicolumn{6}{|c|}{ CENTRAL HARDWOOD } \\
\hline Anoka & 36.6 & 19.5 & 10.6 & 5.6 & 0.9 \\
\hline Benton & 26.1 & 13.1 & 8.3 & 4.5 & 0.2 \\
\hline Carver & 10.4 & 6.6 & 2.4 & 1.2 & 0.2 \\
\hline Chisago & 50.2 & 24.1 & 17.1 & 7.9 & 1.1 \\
\hline Dakota & 16.5 & 7.5 & 6.7 & 1.8 & 0.5 \\
\hline Douglas & 18.5 & 6.5 & 8.0 & 3.5 & 0.5 \\
\hline Filimore & 65.3 & 35.4 & 22.5 & 6.8 & 0.6 \\
\hline Goodhue & 56.4 & 32.2 & 18.6 & 5.1 & 0.5 \\
\hline Hennepin & 7.8 & 4.6 & 1.9 & 0.9 & 0.4 \\
\hline Houston & 111.5 & 71.7 & 29.7 & 9.7 & 0.4 \\
\hline Isanti & 46.9 & 17.8 & 18.7 & 9.2 & 1.2 \\
\hline Kanabec & 129.0 & 41.2 & 68.8 & 17.3 & 1.7 \\
\hline Le Sueur & 10.0 & 5.8 & 2.9 & 1.0 & 0.3 \\
\hline Mille Lacs & 122.8 & 39.1 & 64.6 & 17.2 & 1.9 \\
\hline Morrison & 148.7 & 68.9 & 55.9 & 21.6 & 2.3 \\
\hline 01msted & 32.0 & 18.0 & 10.0 & 3.7 & 0.3 \\
\hline otter Ta $\mathbf{i}$ & 186.3 & 53.3 & 101.0 & 28.6 & 3.4 \\
\hline Pine & 425.6 & 84.9 & 246.1 & 89.0 & 5.6 \\
\hline Ramsey & -- & -- & -- & -- & -- \\
\hline Rice & 12.1 & 7.2 & 3.3 & 1.3 & 0.3 \\
\hline Scott & 13.6 & 8.6 & 3.2 & 1.4 & 0.4 \\
\hline Sherburne & 56.9 & 31.2 & 17.0 & 8.4 & 0.3 \\
\hline Stearns & 56.0 & 33.6 & 15.5 & 6.6 & 0.3 \\
\hline Todd & 104.5 & 37.7 & 47.1 & 17.1 & 2.6 \\
\hline Wabasha & 57.4 & 30.7 & 19.9 & 6.0 & 0.8 \\
\hline Washington & 10.2 & 5.6 & 3.2 & 1.2 & 0.2 \\
\hline Winona & 106.3 & 66.0 & 30.8 & 9.1 & 0.4 \\
\hline Wright & 33.5 & 14.7 & 14.0 & 4.2 & 0.6 \\
\hline All counties & $1,951.1$ & 785.5 & 847.8 & 289.9 & 27.9 \\
\hline
\end{tabular}




\begin{tabular}{|c|c|c|c|c|c|}
\hline & & & & e class & \\
\hline County & $\begin{array}{l}\text { All } \\
\text { stands }\end{array}$ & $\begin{array}{l}\text { Sawtimber } \\
\text { stands }\end{array}$ & $\begin{array}{l}\text { Poletimber } \\
\text { stands }\end{array}$ & $\begin{array}{l}\text { Sapling and } \\
\text { seedling stands }\end{array}$ & $\begin{array}{c}\text { Nonstocked } \\
\text { areas }\end{array}$ \\
\hline Big Stone & 2.4 & 1.0 & 0.7 & 0.7 & -- \\
\hline Blue Earth & 21.4 & 15.3 & 3.3 & 2.5 & 0.3 \\
\hline Brown & 9.1 & 6.5 & 1.5 & 1.0 & 0.1 \\
\hline Chippewa & 3.9 & 1.3 & 1.3 & 1.2 & 0.1 \\
\hline Clay & 10.3 & 3.7 & 3.2 & 3.3 & 0.1 \\
\hline Cottonwood & 2.5 & 1.2 & 0.9 & 0.4 & -- \\
\hline Dodge & 6.4 & 5.0 & 0.9 & 0.4 & 0.1 \\
\hline Faribault & 6.2 & 4.0 & 1.3 & 0.9 & -- \\
\hline Freeborn & 4.8 & 3.3 & 0.8 & 0.6 & 0.1 \\
\hline Grant & 2.9 & 1.4 & 1.1 & 0.4 & -- \\
\hline Jackson & 2.1 & 1.4 & 0.5 & 0.2 & -- \\
\hline Kandiyohi & 9.9 & 6.0 & 2.1 & 1.7 & 0.1 \\
\hline Kittson & 67.5 & 6.3 & 28.0 & 32.5 & 0.7 \\
\hline Lac qui Parle & 4.6 & 2.3 & 1.2 & 1.1 & -- \\
\hline Lincoln & 2.0 & 1.4 & 0.5 & 0.1 & -- \\
\hline Lyon & 4.4 & 2.3 & 1.1 & 0.9 & 0.1 \\
\hline Mcleod & 5.8 & 4.3 & 1.0 & 0.5 & -- \\
\hline Marshall & 128.5 & 16.7 & 47.7 & 62.9 & 1.2 \\
\hline Martin & 3.6 & 2.0 & 1.1 & 0.4 & 0.1 \\
\hline Meeker & 10.8 & 8.8 & 0.7 & 1.0 & 0.3 \\
\hline Mower & 5.2 & 4.0 & 0.8 & 0.3 & 0.1 \\
\hline Murray & 1.1 & 0.6 & 0.3 & 0.2 & -- \\
\hline Nicollet & 12.3 & 9.3 & 1.4 & 1.3 & 0.3 \\
\hline Nobles & 0.7 & 0.3 & 0.3 & 0.1 & -- \\
\hline Norman & 22.2 & 6.4 & 9.6 & 5.9 & 0.3 \\
\hline Pennington & 30.7 & 6.3 & 14.3 & 9.9 & 0.2 \\
\hline Pipestone & 0.4 & 0.2 & 0.2 & -- & -- \\
\hline Polk & 68.0 & 20.7 & 26.1 & 20.5 & 0.7 \\
\hline Pope & 6.9 & 2.4 & 2.4 & 2.0 & 0.1 \\
\hline Red Lake & 27.2 & 5.3 & 13.0 & 8.7 & 0.2 \\
\hline Redwood & 6.2 & 3.1 & 1.8 & 1.2 & 0.1 \\
\hline Renville & 7.2 & 4.5 & 1.5 & 1.1 & 0.1 \\
\hline Rock & 0.5 & 0.2 & 0.2 & 0.1 & -- \\
\hline Sibley & 10.9 & 8.0 & 1.8 & 1.0 & 0.1 \\
\hline Steele & 5.0 & 3.6 & 0.8 & 0.5 & 0.1 \\
\hline Stevens & 1.0 & 0.4 & 0.4 & 0.2 & -- \\
\hline Swift & 4.7 & 2.0 & 1.6 & 1.0 & 0.1 \\
\hline Traverse & 0.9 & 0.3 & 0.3 & 0.3 & -- \\
\hline Waseca & 4.6 & 3.5 & 0.5 & 0.5 & 0.1 \\
\hline Wa tonwan & 1.1 & 0.6 & 0.2 & 0.3 & -- \\
\hline Wilkin & 0.5 & 0.1 & 0.1 & 0.3 & -- \\
\hline Yellow Medicine & 7.8 & 4.6 & 2.2 & 0.9 & 0.1 \\
\hline All counties & 534.2 & 180.6 & 178.7 & 169.0 & 5.9 \\
\hline All units & $13,695.1$ & $3,134.8$ & $6,956.1$ & $3,434.8$ & 169.4 \\
\hline
\end{tabular}


Table 13.--Area of commercial forest land by forest type, stand-size class, and site class, Minnesota, 1977

(In thousand acres)

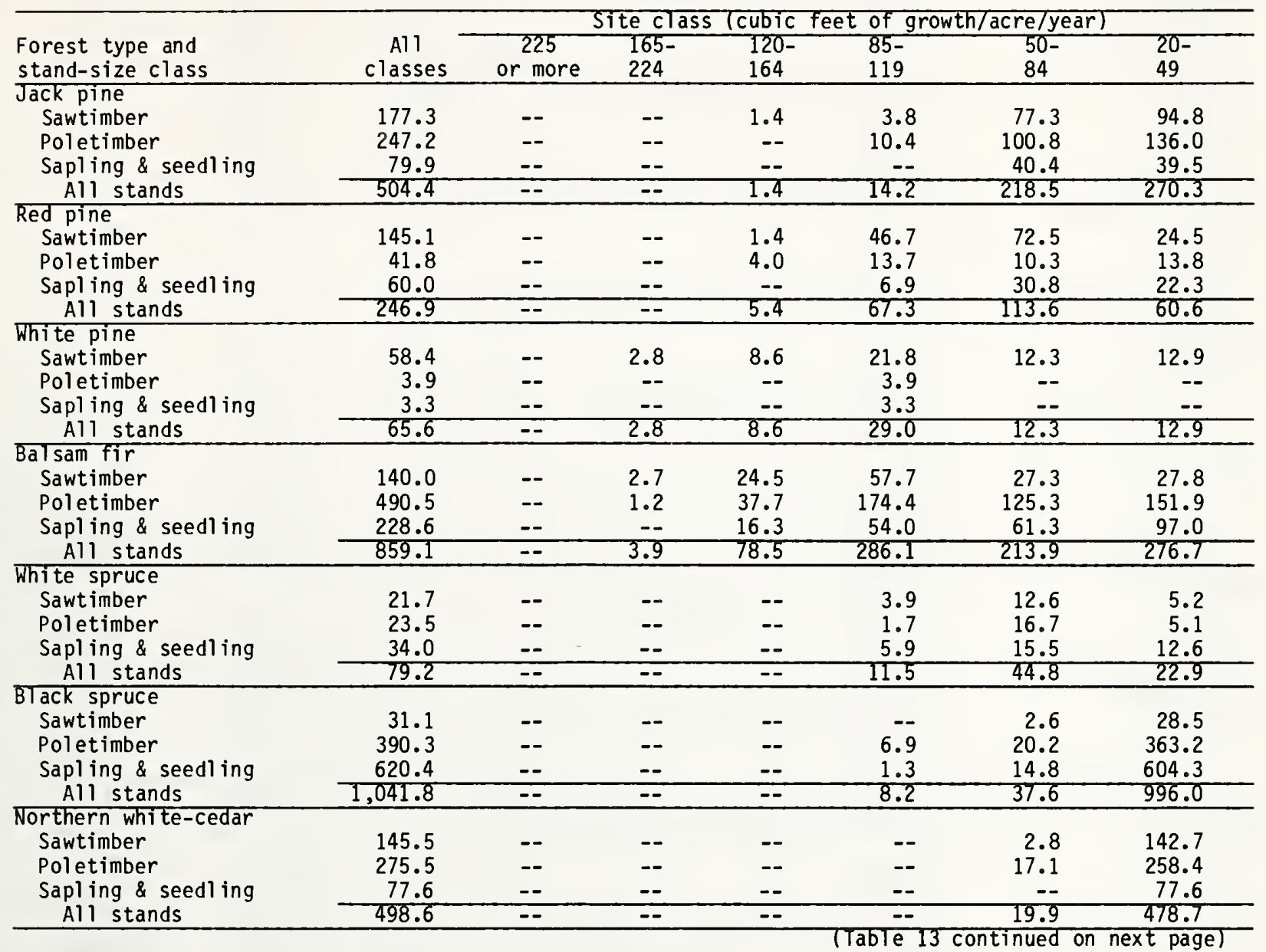


(Table 13 continued)

\begin{tabular}{|c|c|c|c|c|c|c|c|}
\hline \multirow{2}{*}{$\begin{array}{l}\text { Forest type and } \\
\text { stand-size class }\end{array}$} & \multirow[b]{2}{*}{$\begin{array}{c}\text { Al1 } \\
\text { classes }\end{array}$} & \multicolumn{3}{|c|}{ Site class (cubic } & \multicolumn{3}{|c|}{ eet of growth/acre/year) } \\
\hline & & $\begin{array}{c}225 \\
\text { or more }\end{array}$ & $\begin{array}{l}165- \\
224\end{array}$ & $\begin{array}{l}120- \\
164\end{array}$ & $\begin{array}{l}85- \\
119\end{array}$ & $\begin{array}{l}50- \\
84\end{array}$ & $\begin{array}{l}20- \\
49\end{array}$ \\
\hline \multicolumn{8}{|l|}{ Tamarack } \\
\hline Sawt imber & 43.5 & -- & -- & -- & 1.3 & 12.1 & 30.1 \\
\hline Poletimber & 200.3 & -- & -- & -- & 2.0 & 33.6 & 164.7 \\
\hline Sapling \& seedling & 221.6 & -- & -- & -- & - & 19.4 & 202.2 \\
\hline All stands & 465.4 & -- & -- & -- & 3.3 & 65.1 & 397.0 \\
\hline \multicolumn{8}{|l|}{ Oak-hickory } \\
\hline Sawtimber & 452.7 & -- & -- & 2.8 & 10.0 & 134.6 & 305.5 \\
\hline Poletimber & 360.9 & -- & -- & - & 14.7 & 118.8 & 227.4 \\
\hline Sapling \& seedling & 80.3 & -- & - & -- & - & 20.7 & 59.6 \\
\hline All stands & 893.9 & -- & -- & 2.8 & 24.7 & 274.1 & 592.3 \\
\hline \multicolumn{8}{|l|}{ ETm-ash-cottonwood } \\
\hline Sawtimber & 194.8 & -- & -- & -- & 6.3 & 37.2 & 151.3 \\
\hline Poletimber & 388.8 & -- & -- & -- & 1.1 & 25.8 & 361.9 \\
\hline Sapl ing \& seedling & 154.5 & -- & - & - & -. & 4.6 & 149.9 \\
\hline All stands & 738.1 & -- & -- & -- & 7.4 & 67.6 & 663.1 \\
\hline \multicolumn{8}{|l|}{ Maple-basswood } \\
\hline Sawtimber & 675.6 & -- & -- & -- & 30.6 & 245.2 & 399.8 \\
\hline Poletimber & 493.5 & -- & - & - & 29.4 & 130.6 & 333.5 \\
\hline Sapling \& seedling & 114.8 & -- & -- & -- & 1.1 & 23.1 & 90.6 \\
\hline All stands & $1,283.9$ & -- & -- & $=-$ & 61.1 & 398.9 & 823.9 \\
\hline \multicolumn{8}{|l|}{ Aspen } \\
\hline Sawtimber & 791.4 & -- & -- & 27.3 & 253.4 & 440.8 & 69.9 \\
\hline Poletimber & $3,032.3$ & -- & -- & 28.6 & 985.0 & $1,614.6$ & 404.1 \\
\hline Sapl ing \& seedling & $1,478.6$ & -- & -- & 4.2 & 210.0 & 828.0 & 436.4 \\
\hline All stands & $5,302.3$ & -- & -- & 60.1 & $1,448.4$ & $2,883.4$ & 910.4 \\
\hline \multicolumn{8}{|l|}{ Paper birch } \\
\hline Sawtimber & 142.0 & -- & -- & -- & 2.9 & 51.1 & 88.0 \\
\hline Poletimber & 758.6 & -- & -- & 1.4 & 6.7 & 259.0 & 491.5 \\
\hline Sapling \& seedling & 97.0 & -- & -- & -- & 1.3 & 22.1 & 73.6 \\
\hline All stands & 997.6 & -- & $=-$ & 1.4 & 10.9 & 332.2 & 653.1 \\
\hline \multicolumn{8}{|l|}{ Batsam poptar } \\
\hline Sawtimber & 115.7 & -- & -- & -- & 22.3 & 69.5 & 23.9 \\
\hline Poletimber & 249.0 & -- & -- & 1.4 & 34.3 & 126.5 & 86.8 \\
\hline Sapling \& seedling & 184.2 & -- & -- & -- & 10.1 & 90.0 & 84.1 \\
\hline All stands & 548.9 & -- & -- & 1.4 & 66.7 & 286.0 & 194.8 \\
\hline Nonstocked & 169.4 & -- & -- & -- & 5.5 & 31.0 & 132.9 \\
\hline \multicolumn{8}{|l|}{ AाT types } \\
\hline Sawt imber & $3,134.8$ & -- & 5.5 & 66.0 & 460.7 & $1,197.9$ & $1,404.7$ \\
\hline Poletimber & $6,956.1$ & -- & 1.2 & 73.1 & $1,284.2$ & $2,599.3$ & $2,998.3$ \\
\hline Sapl ing \& seedling & $3,434.8$ & -- & - & 20.5 & 293.9 & $1,170.7$ & $1,949.7$ \\
\hline Nonstocked & 169.4 & -- & -- & - & 5.5 & 31.0 & 132.9 \\
\hline All stands & $13,695,1$ & -- & 6.7 & 159.6 & $2,044,3$ & $4,998.9$ & $6,485.6$ \\
\hline
\end{tabular}




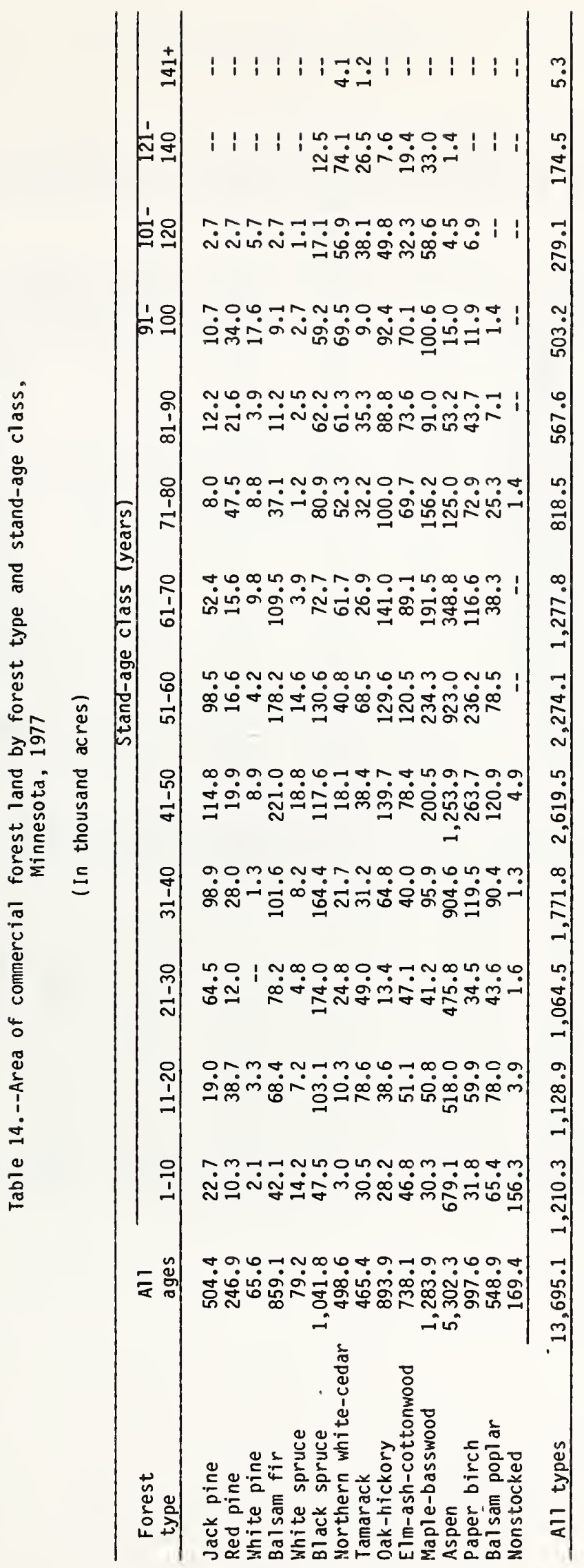


Table 15.--Area of commercial forest land by forest type and area-condition class, Minnesota, 1977

(In thousand acres)

\begin{tabular}{|c|c|c|c|c|c|c|c|c|}
\hline \multirow{2}{*}{$\begin{array}{l}\text { Forest } \\
\text { type }\end{array}$} & \multirow{2}{*}{$\begin{array}{c}\text { AlT } \\
\text { classes } \\
\end{array}$} & \multicolumn{7}{|c|}{ Area-condition class } \\
\hline & & 10 & 20 & 30 & 40 & 50 & 60 & 70 \\
\hline Jack pine & 504.4 & -- & -- & -- & -- & 154.0 & 311.8 & 38.6 \\
\hline Red pine & 246.9 & -- & -- & -- & -- & 118.5 & 116.1 & 12.3 \\
\hline White pine & 65.6 & -- & -- & -- & -- & 25.7 & 35.2 & 4.7 \\
\hline Bal sam fir & 859.1 & -- & -- & -- & -- & 225.7 & 497.6 & 135.8 \\
\hline White spruce & 79.2 & -- & -- & -- & -- & 17.8 & 44.3 & 17.1 \\
\hline Black spruce & $1,041.8$ & -- & -- & -- & -- & 367.9 & 519.7 & 154.2 \\
\hline Northern white-cedar & 498.6 & -- & -- & -- & -- & 196.0 & 234.9 & 67.7 \\
\hline Tamarack & 465.4 & -- & -- & -- & -- & 75.6 & 230.4 & 159.4 \\
\hline Oak-hickory & 893.9 & -- & -- & -- & -- & 227.5 & 495.9 & 170.5 \\
\hline Elm-ash-cottonwood & 738.1 & -- & -- & -- & -- & 189.6 & 322.0 & 226.5 \\
\hline Maple-basswood & $1,283.9$ & -- & -- & -- & -- & 285.0 & 685.5 & 313.4 \\
\hline Aspen & $5,302.3$ & 1.3 & -- & -- & -- & $1,255.4$ & $3,050.2$ & 995.4 \\
\hline Paper birch & 997.6 & -- & -- & -- & -- & 238.6 & 600.9 & 158.1 \\
\hline Bal sam poplar & 548.9 & -- & -- & -- & -- & 128.5 & 265.2 & 155.2 \\
\hline Nonstocked & 169.4 & -- & -- & -- & -- & -- & 20.2 & 149.2 \\
\hline All types & $13,695.1$ & 1.3 & -- & -- & -- & $3,505.8$ & $7,429.9$ & $2,758.1$ \\
\hline
\end{tabular}


Table 16.--Area of commercial forest land by forest type, site-index class, and Forest Survey Unit, Minnesota, 1977

(In thousand acres)

ALL UNITS

\begin{tabular}{|c|c|c|c|c|c|c|c|c|c|c|}
\hline $\begin{array}{l}\text { Forest } \\
\text { type }\end{array}$ & AाT & $\pi-20$ & $21-30$ & $31-40$ & $\frac{\text { Sit }}{41-50}$ & index cT & $3 s$ (feet) & $7=80$ & वा 0 & 91 \\
\hline Jack pine & 504.4 & -- & -- & 18.2 & 112.4 & 194.3 & 132.7 & $\frac{11-80}{40.0}$ & $\frac{81-90}{5.4}$ & $\frac{91+}{1.4}$ \\
\hline Red pine & 246.9 & -- & -- & 5.8 & 36.4 & 118.2 & 42.7 & 43.8 & -- & -- \\
\hline White pine & 65.6 & -- & -- & 3.7 & 18.8 & 29.5 & 10.7 & 2.9 & - & -- \\
\hline Balsam fir & 859.1 & -- & 18.6 & 179.3 & 316.6 & 250.4 & 71.2 & 19.1 & 3.9 & -- \\
\hline White spruce & 79.2 & -- & -- & 5.8 & 32.7 & 23.8 & 16.9 & -- & - & -- \\
\hline Black spruce & $1,041.8$ & 27.2 & 369.8 & 375.1 & 223.9 & 37.6 & 6.9 & 1.3 & -- & -- \\
\hline Northern white-cedar & 498.6 & 85.8 & 278.0 & 95.2 & 25.7 & 10.0 & 3.9 & -- & -- & -- \\
\hline Tamarack & 465.4 & 4.5 & 91.6 & 168.1 & 132.8 & 52.3 & 12.8 & 3.3 & -- & -- \\
\hline 0ak-hickory & 893.9 & -- & 7.5 & 164.3 & 307.2 & 225.7 & 134.9 & 39.5 & 12.0 & 2.8 \\
\hline Elm-ash-cottonwood & 738.1 & -- & 3.2 & 136.3 & 218.8 & 259.3 & 82.5 & 30.6 & 5.9 & 1.5 \\
\hline Maple-basswood & $1,283.9$ & -- & -- & 63.4 & 303.0 & 465.1 & 316.5 & 103.8 & 32.1 & -- \\
\hline Aspen & $5,302.3$ & 1.4 & 12.3 & 73.1 & 397.7 & $1,225.1$ & $1,780.0$ & $1,286.2$ & 466.4 & 60.1 \\
\hline Paper birch & 997.6 & -- & 3.0 & 47.0 & 186.7 & 388.9 & 290.1 & 69.5 & 11.0 & 1.4 \\
\hline Balsam poplar & 548.9 & -- & -- & 25.4 & 111.2 & 134.1 & 169.9 & 73.7 & 30.4 & 4.2 \\
\hline Nonstocked & 169.4 & 4.3 & 20.8 & 31.1 & 51.1 & 36.1 & 20.6 & 5.4 & -- & -- \\
\hline All types & $13,695.1$ & 123.2 & 804.8 & $1,391.8$ & $2,475.0$ & $3,450.4$ & $3,092.3$ & $1,719.1$ & 567.1 & 71.4 \\
\hline & & & & ASPEN & IRCH & & & & & \\
\hline Jack pine & 165.7 & -- & -- & 5.4 & 55.9 & 78.5 & 20.7 & 5.2 & -- & -- \\
\hline Red pine & 114.4 & -- & -- & 1.3 & 22.1 & 66.1 & 21.0 & 3.9 & -- & -- \\
\hline White pine & 33.8 & -- & -- & 3.7 & 15.0 & 11.6 & 3.5 & - & -- & -- \\
\hline Balsam fir & 626.0 & -- & 11.0 & 106.0 & 245.6 & 195.0 & 56.2 & 9.5 & 2.7 & -- \\
\hline White spruce & 60.1 & -- & -- & 5.8 & 21.7 & 21.0 & 11.6 & -- & -- & -- \\
\hline Black spruce & 745.7 & 21.6 & 248.3 & 270.1 & 169.9 & 29.2 & 5.3 & 1.3 & -- & -- \\
\hline Northern white-cedar & 317.8 & 52.0 & 182.9 & 54.4 & 17.9 & 6.7 & 3.9 & -- & -- & -- \\
\hline Tamarack & 157.6 & 1.2 & 40.8 & 51.5 & 44.4 & 17.0 & 2.7 & -- & -- & -- \\
\hline Oak-hickory & 5.4 & -- & -- & -- & 1.2 & 1.4 & 1.4 & 1.4 & -- & -- \\
\hline Elm-ash-cottonwood & 243.7 & -- & -- & 57.6 & 97.2 & 75.4 & 10.5 & 3.0 & -- & -- \\
\hline Maple-basswood & 214.5 & -- & -- & 14.2 & 75.0 & 82.6 & 39.0 & 2.7 & 1.0 & -- \\
\hline Aspen & $1,947.4$ & -- & 8.9 & 20.3 & 156.0 & 530.7 & 698.2 & 391.8 & 118.9 & 22.6 \\
\hline Paper birch & 546.3 & -- & 1.4 & 36.7 & 112.0 & 223.5 & 144.0 & 27.2 & 1.5 & -- \\
\hline Bal sam poplar & 208.0 & -- & - & 8.6 & 28.8 & 52.2 & 64.2 & 33.4 & 18.0 & 2.8 \\
\hline Nonstocked & 65.0 & -- & 3.5 & 9.3 & 33.7 & 7.2 & 8.4 & 2.9 & -- & -- \\
\hline All types &,, 451.4 & 74.8 & 496.8 & 644.9 & $1,096.4$ & $1,398.1$ & $1,090.6$ & 482.3 & 142.1 & 25.4 \\
\hline & & & & NORTHE & PÍNE & & & & & \\
\hline Jack pine & 327.4 & -- & -- & 12.8 & 54.6 & 112.7 & 109.8 & 30.7 & 5.4 & 1.4 \\
\hline Red pine & 121.9 & -- & -- & 4.5 & 14.3 & 47.4 & 17.7 & 38.0 & - & - \\
\hline White pine & 24.8 & -- & -- & -- & 2.5 & 15.3 & 5.6 & 1.4 & -- & - \\
\hline Balsam fir & 225.6 & -- & 7.6 & 71.3 & 69.6 & 51.3 & 15.0 & 9.6 & 1.2 & -- \\
\hline White spruce & 19.1 & -- & -- & -- & 11.0 & 2.8 & 5.3 & - & -- & -- \\
\hline Black spruce & 278.2 & 5.6 & 120.0 & 91.5 & 51.1 & 8.4 & 1.6 & -- & -- & - \\
\hline Nor thern white-cedar & 180.8 & 33.8 & 95.1 & 40.8 & 7.8 & 3.3 & -- & -- & -- & -- \\
\hline Tamarack & 276.0 & 3.3 & 49.1 & 113.2 & 71.1 & 30.7 & 7.3 & 1.3 & -- & -- \\
\hline Oak-hickory & 247.4 & -- & 4.6 & 34.9 & 92.8 & 67.0 & 34.5 & 9.4 & 4.2 & - \\
\hline Elm-ash-cottonwood & 309.8 & -- & 1.4 & 56.3 & 82.6 & 119.1 & 39.2 & 10.1 & 1.1 & -- \\
\hline Maple-basswood & 466.4 & -- & -- & 21.7 & 100.1 & 171.9 & 116.8 & 39.9 & 16.0 & -- \\
\hline Aspen & $2,541.7$ & 1.4 & 1.8 & 36.9 & 139.9 & 440.0 & 808.1 & 743.0 & 333.1 & 37.5 \\
\hline Paper birch & 371.2 & -- & -- & 4.9 & 54.8 & 143.1 & 122.0 & 35.5 & 9.5 & 1.4 \\
\hline Balsam poplar & 297.5 & -- & -- & 12.3 & 65.5 & 68.6 & 98.5 & 38.8 & 12.4 & 1.4 \\
\hline Nonstocked & 70.6 & 4.3 & 15.8 & 11.8 & 10.3 & 18.1 & 7.8 & 2.5 & -- & -- \\
\hline All types & $5,758.4$ & 48.4 & 295.4 & 512.9 & 828.0 & $1,299.7$ & $1,389.2$ & 960.2 & 382.9 & 41.7 \\
\hline
\end{tabular}


(Table 16 continued)

CENTRAL HARDWOODS

\begin{tabular}{|c|c|c|c|c|c|c|c|c|c|c|}
\hline Forest & AाT & $7-30$ & 21-30 & $31-4$ & Site & dexcl & (feet) & $7-80$ & $81-00$ & OIt \\
\hline Jack pine & 11.3 & $\frac{11-<0}{--}$ & $\frac{21-30}{--}$ & $\frac{31-40}{--}$ & $\frac{41-50}{1.9}$ & $\frac{51-60}{3.1}$ & $\frac{01-10}{2.2}$ & $\frac{11-00}{4.1}$ & $01-90$ & $\frac{91+}{--}$ \\
\hline Red pine & 10.6 & -- & -- & -- & -- & 4.7 & 4.0 & 1.9 & -- & -- \\
\hline White pine & 7.0 & -- & -- & -- & 1.3 & 2.6 & 1.6 & 1.5 & -- & -- \\
\hline Balsam fir & 7.5 & -- & -- & 2.0 & 1.4 & 4.1 & -- & -- & -- & -- \\
\hline White spruce & -- & -- & -- & -- & -- & -- & -- & -- & -- & -- \\
\hline Black spruce & 17.9 & -- & 1.5 & 13.5 & 2.9 & -- & -- & - & -- & - \\
\hline Northern white-cedar & -- & -- & -- & -- & -- & -- & -- & -- & -- & -- \\
\hline Tamarack & 30.8 & -- & 0.7 & 3.4 & 17.3 & 4.6 & 2.8 & 2.0 & -- & -- \\
\hline Oak-hickory & 571.8 & -- & 1.6 & 98.2 & 195.2 & 144.6 & 92.9 & 28.7 & 7.8 & 2.8 \\
\hline Elm-ash-cottonwood & 129.5 & -- & 1.8 & 13.5 & 27.3 & 45.8 & 21.3 & 15.0 & 4.8 & -- \\
\hline Maple-basswood & 485.8 & -- & -- & 20.5 & 105.3 & 167.6 & 135.0 & 43.8 & 13.6 & -- \\
\hline Aspen & 560.2 & -- & 1.6 & 2.5 & 36.2 & 153.1 & 223.6 & 128.8 & 14.4 & - \\
\hline Paper birch & 80.1 & -- & 1.6 & 5.4 & 19.9 & 22.3 & 24.1 & 6.8 & -- & -- \\
\hline Balsam poplar & 10.7 & -- & -- & 1.5 & 2.9 & 3.2 & 1.6 & 1.5 & -- & -- \\
\hline Nonstocked & 27.9 & -- & 1.5 & 10.0 & 2.6 & 9.4 & 4.4 & -- & - & -- \\
\hline All types & $1,951.1$ & $=$ & 10.3 & 170.5 & 414.2 & 565.1 & 513.5 & 234.1 & 40.6 & 2.8 \\
\hline \multicolumn{11}{|c|}{ PRAIRIE } \\
\hline Jack pine & -- & -- & - & $=-$ & $=$ & $=-$ & -- & -- & -- & -- \\
\hline Red pine & -- & -- & -- & -- & -- & -- & -- & -- & - & - \\
\hline White pine & -- & -- & -- & -- & -- & -- & -- & -- & -- & -- \\
\hline Balsam fir & -- & -- & - & $\rightarrow$ & -- & -- & $\rightarrow$ & -- & -- & -- \\
\hline White spruce & -- & -- & - & -- & -- & -- & -- & -- & -- & -- \\
\hline Black spruce & -- & -- & -- & -- & -- & -- & -- & -- & -- & -- \\
\hline Northern white-cedar & -- & -- & -- & -- & -- & -- & -- & -- & -- & - \\
\hline Tamarack & 1.0 & -- & 1.0 & -- & -- & - & -- & - & -- & -- \\
\hline Oak-hickory & 69.3 & -- & 1.3 & 31.2 & 18.0 & 12.7 & 6.1 & -- & -- & -- \\
\hline Elm-ash-cottonwood & 55.1 & -- & - & 8.9 & 11.7 & 19.0 & 11.5 & 2.5 & -- & 1.5 \\
\hline Maple-basswood & 117.2 & -- & -- & 7.0 & 22.6 & 43.0 & 25.7 & 17.4 & 1.5 & -- \\
\hline Aspen & 253.0 & $\ldots$ & -- & 13.4 & 65.6 & 101.3 & 50.1 & 22.6 & -- & -- \\
\hline Paper birch & -- & -- & -- & -- & -- & -- & -- & -- & -- & -- \\
\hline Balsam poplar & 32.7 & -- & -- & 3.0 & 14.0 & 10.1 & 5.6 & -- & -. & -- \\
\hline Nonstocked & 5.9 & -- & -- & -- & 4.5 & 1.4 & - & -- & -- & -- \\
\hline All types & 534.2 & $=$ & 2.3 & 63.5 & 136.4 & 187.5 & 99.0 & 42.5 & 1.5 & 1.5 \\
\hline
\end{tabular}




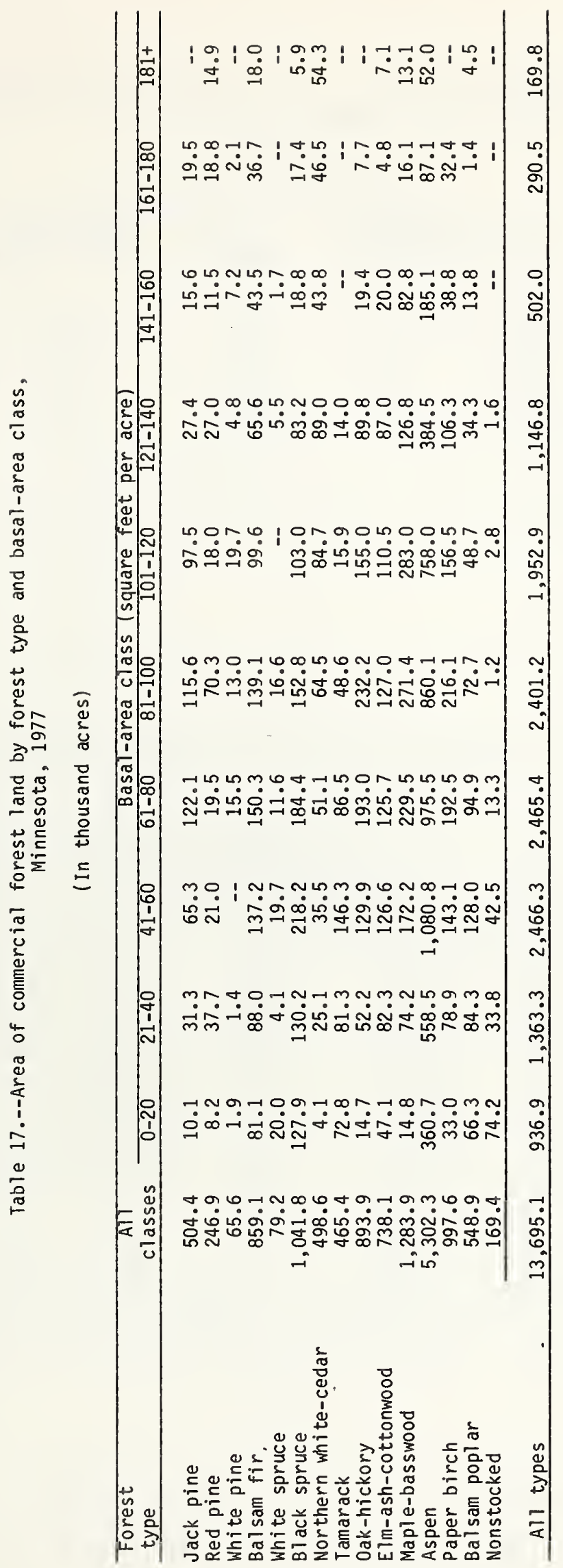


Table 18.--Area of commercial forest land by stocking class of growing-stock trees and stand-size class, Minnesota, 1977

(In thousand acres)

\begin{tabular}{|c|c|c|c|c|c|}
\hline \multirow[b]{2}{*}{$\begin{array}{l}\text { Percent stocking } \\
\text { class }\end{array}$} & \multirow[b]{2}{*}{$\begin{array}{c}\text { Al1 } \\
\text { stands }\end{array}$} & \multicolumn{4}{|c|}{ Stand-size class } \\
\hline & & $\begin{array}{c}\text { Sawt imber } \\
\text { stands }\end{array}$ & $\begin{array}{c}\text { Poletimber } \\
\text { stands }\end{array}$ & $\begin{array}{c}\text { Sapling and } \\
\text { Seedling stands }\end{array}$ & $\begin{array}{c}\text { Nonstocked } \\
\text { areas }\end{array}$ \\
\hline $\begin{array}{l}\text { Less than } 18 \\
18 \text { to } 60 \\
61 \text { to } 100 \\
101 \text { to } 133 \\
134 \text { or more }\end{array}$ & $\begin{array}{r}165.5 \\
2,725.3 \\
7,480.7 \\
3,006.1 \\
317.5 \\
\end{array}$ & $\begin{array}{r}1.6 \\
614.4 \\
1,734.3 \\
715.5 \\
69.0 \\
\end{array}$ & $\begin{array}{r}7.3 \\
1,213.7 \\
3,924.0 \\
1,660.3 \\
150.8 \\
\end{array}$ & $\begin{array}{r}7.4 \\
897.2 \\
1,802.2 \\
630.3 \\
97.7 \\
\end{array}$ & $\begin{array}{r}149.2 \\
-- \\
20.2 \\
-- \\
-- \\
\end{array}$ \\
\hline All classes & $13,695.1$ & $3,134.8$ & $6,956.1$ & $3,434.8$ & 169.4 \\
\hline
\end{tabular}

Table 19.--Area of commercial forest land by stocking class based on selected stand components, Minnesota, 1977

(In thousand acres)

\begin{tabular}{|c|c|c|c|c|c|}
\hline \multirow{3}{*}{$\begin{array}{l}\text { Stocking } \\
\text { percentage }\end{array}$} & \multirow{3}{*}{$\begin{array}{c}\text { All } \\
\text { trees }\end{array}$} & \multicolumn{4}{|c|}{ Stocking classified in terms of } \\
\hline & & & owing-stock & rees & Rough and \\
\hline & & Total & Desirable & Acceptable & rotten trees \\
\hline $0-10$ & 15.6 & 69.2 & $13,294.5$ & 73.3 & $4,846.2$ \\
\hline $11-20$ & 49.7 & 165.3 & 279.2 & 172.5 & $3,350.3$ \\
\hline $21-30$ & 104.8 & 315.9 & 87.0 & 319.6 & $3,308.0$ \\
\hline $31-40$ & 202.3 & 550.1 & 16.0 & 592.4 & $1,170.5$ \\
\hline $41-50$ & 376.4 & 690.8 & 11.5 & 731.5 & 553.9 \\
\hline $51-60$ & 520.8 & $1,098.9$ & 5.6 & $2,302.3$ & 270.7 \\
\hline $61-70$ & 661.3 & $2,456.3$ & -- & $1,396.3$ & 117.5 \\
\hline $71-80$ & $1,003.9$ & $1,570.4$ & -- & $1,645.8$ & 42.8 \\
\hline $81-90$ & $1,299.4$ & $1,725.9$ & -- & $1,721,4$ & 15.0 \\
\hline $91-100$ & $2,823.3$ & $1,729.4$ & -- & $1,677.6$ & 2.4 \\
\hline $101-110$ & $1,788.3$ & $1,299.1$ & 1.3 & $1,229.0$ & 9.4 \\
\hline $111-120$ & $1,739.0$ & 973.4 & -- & 900.1 & 4.2 \\
\hline $121-130$ & $1,432.6$ & 613.4 & -- & 553.3 & 1.3 \\
\hline $131-140$ & 979.3 & 277.0 & -- & 238.2 & -- \\
\hline $141-150$ & 513.5 & 115.6 & -- & 100.3 & 2.9 \\
\hline $151-160$ & 184.9 & 44.4 & -- & 41.5 & -- \\
\hline $161+$ & -- & -- & -- & -- & -- \\
\hline Total & $13,695.1$ & $13,695.1$ & $13,695.1$ & $13,695.1$ & $13,695.1$ \\
\hline
\end{tabular}


Table 20.--Area of noncommercial forest land by ownership class, Minnesota, 1977

(In thousand acres)

\begin{tabular}{lrrr}
\hline Ownership class & $\begin{array}{r}\text { AlT } \\
\text { areas }\end{array}$ & $\begin{array}{r}\text { Productive- } \\
\text { reserved areas }\end{array}$ & $\begin{array}{c}\text { Unproductive } \\
\text { areas }\end{array}$ \\
\hline National forest & 884.3 & 758.7 & 125.6 \\
Other federal & 253.8 & 126.7 & 127.1 \\
State, county and municipal & $1,560.3$ & 281.6 & $1,278.7$ \\
Forest industry & 35.2 & -- & 35.2 \\
Farmer & 211.1 & -- & 211.1 \\
Miscellaneous private & 69.4 & 11.6 & 57.8 \\
\hline
\end{tabular}

$1 /$ Includes 3,000 acres of productive-deferred forest land, commercial forest 1 and being withheld from harvest while a decision is made whether to place it in productive-reserved status.

Table 21.--Area of noncommercial forest 1 and by forest type, Minnesota, 1977

(In thousand acres)

\begin{tabular}{lrrr}
\hline $\begin{array}{l}\text { Forest } \\
\text { type }\end{array}$ & $\begin{array}{c}\text { A11 } \\
\text { areas }\end{array}$ & $\begin{array}{r}\text { Productive- } \\
\text { reserved areas }\end{array}$ & $\begin{array}{c}\text { Unproductive } \\
\text { areas }\end{array}$ \\
\hline Jack pine & 211.0 & 208.2 & 2.8 \\
Red-white pine & 79.4 & 71.9 & 7.5 \\
Spruce-fir & 171.9 & 104.7 & 67.2 \\
Black spruce & $1,101.1$ & 68.7 & $1,032.4$ \\
Northern white-cedar & 148.6 & 12.6 & 136.0 \\
Tamarack & 177.0 & 3.2 & 173.8 \\
Oak-hickory & 111.6 & 11.4 & 100.2 \\
Elm-ash-cottonwood & 225.9 & 38.3 & 187.6 \\
Maple-basswood & 30.7 & 23.1 & 7.6 \\
Aspen-birch & 743.0 & 633.9 & 109.1 \\
Nonstocked & 13.9 & 2.6 & 11.3 \\
& & & $1,835.5$ \\
\hline
\end{tabular}

$1 /$ Includes 3,000 acres of productive-deferred forest land, commercial forest land being withheld from harvest while a decision is made whether to $\mathrm{place}$ it in productive-reserved status. 


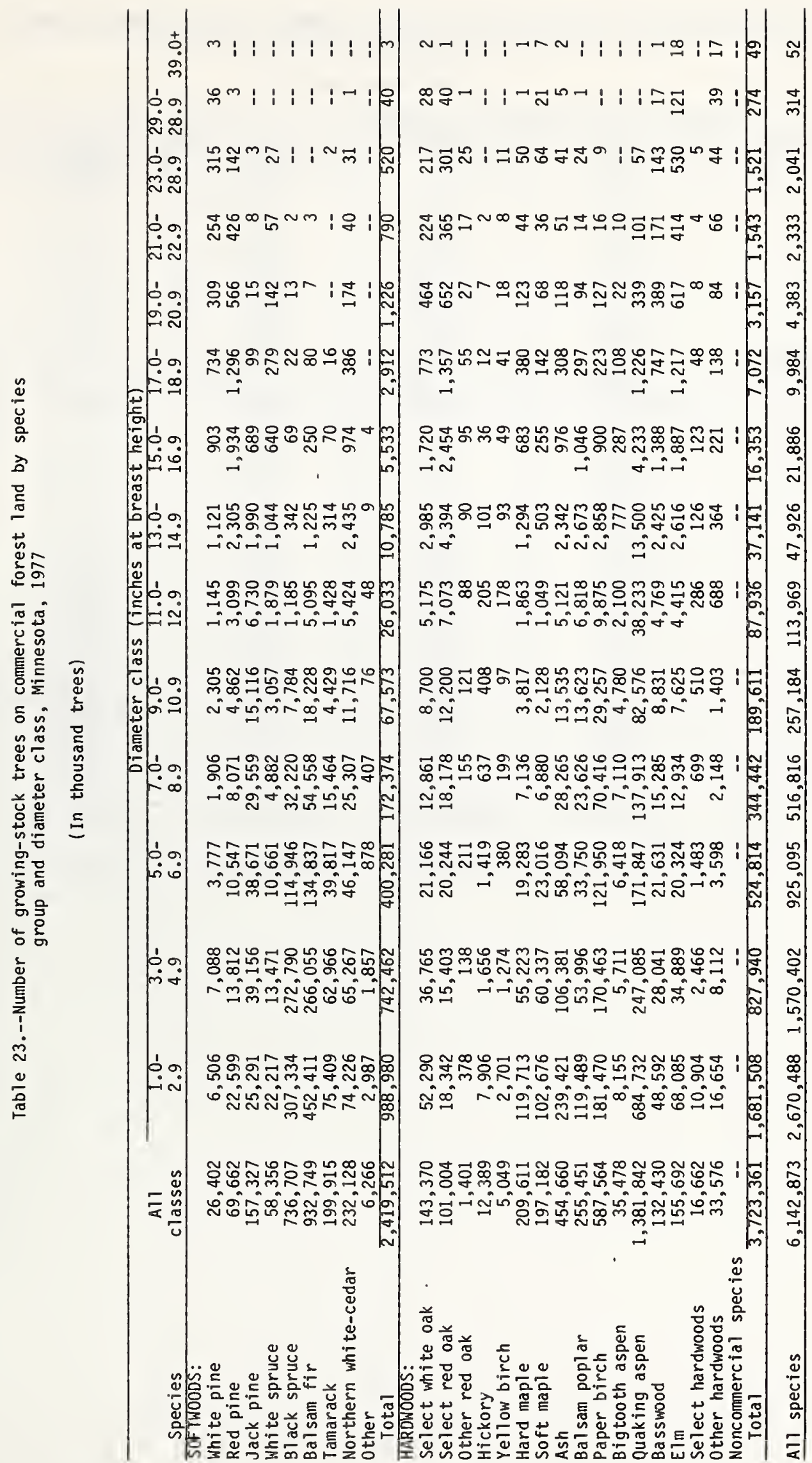


Table 24.--Number of growing-stock trees on commercial forest land by species group and Forest Survey Unit, Minnesota, 1977

(In thousand trees)

\begin{tabular}{|c|c|c|c|c|c|}
\hline Species & $\begin{array}{l}\text { All } \\
\text { units }\end{array}$ & $\begin{array}{l}\text { Aspen- } \\
\text { Birch }\end{array}$ & $\begin{array}{l}\text { Northern } \\
\text { Pine }\end{array}$ & $\begin{array}{l}\text { Central } \\
\text { Hardwood }\end{array}$ & Prairie \\
\hline \multicolumn{6}{|l|}{ SOFTWOODS: } \\
\hline White pine & 26,402 & 12,368 & 11,705 & 2,329 & -- \\
\hline Red pine & 69,662 & 31,991 & 29,529 & 8,123 & 19 \\
\hline Jack pine & 157,327 & 35,364 & 116,948 & 4,972 & 43 \\
\hline White spruce & 58,356 & 36,230 & 21,289 & 798 & 39 \\
\hline Black spruce & 736,707 & 550,365 & 177,347 & 8,811 & 184 \\
\hline Balsam fir & 932,749 & 668,902 & 258,386 & 5,269 & 192 \\
\hline Tamarack & 199,915 & 84,419 & 105,253 & 10,065 & 178 \\
\hline Northern white-cedar & 232,128 & 154,502 & 77,626 & & -- \\
\hline Other softwoods & 6,266 & 315 & 106 & 4,484 & 1,361 \\
\hline Total & $2,419,512$ & $1,574,456$ & 798,189 & 44,851 & 2,016 \\
\hline \multicolumn{6}{|l|}{ HARDWOODS: } \\
\hline Select white oak & 143,370 & 2,529 & 89,517 & 36,550 & 14,774 \\
\hline Select red oak & 101,004 & 1,855 & 57,252 & 39,823 & 2,074 \\
\hline Other red oak & 1,401 & -- & 718 & 638 & 45 \\
\hline Hickory & 12,389 & -- & 198 & 12,111 & 80 \\
\hline Yellow birch & 5,049 & 1,819 & 2,133 & 1,097 & -- \\
\hline Hard maple & 209,611 & 65,281 & 105,767 & 36,150 & 2,413 \\
\hline Soft maple & 197,182 & 85,414 & 71,241 & 40,251 & 276 \\
\hline Ash & 454,660 & 191,103 & 201,976 & 50,525 & 11,056 \\
\hline Bal sam poplar & 255,451 & 97,790 & 135,790 & 3,916 & 17,955 \\
\hline Paper birch & 587,564 & 328,755 & 204,134 & 53,304 & 1,371 \\
\hline Bigtooth aspen & 35,478 & 4,433 & 25,339 & 5,692 & 14 \\
\hline Quaking aspen & $1,381,842$ & 584,299 & 602,327 & 110,801 & 84,415 \\
\hline Basswood & 132,430 & 9,063 & 74,343 & 44,692 & 4,332 \\
\hline Elm & 155,692 & 12,720 & 69,716 & 58,588 & 14,668 \\
\hline Select hardwoods & 16,662 & 312 & 3,140 & 10,271 & 2,939 \\
\hline Other hardwoods & 33,576 & 1,130 & 3,457 & 13,667 & 15,322 \\
\hline \multirow{2}{*}{$\begin{array}{l}\text { Noncommercial species } \\
\text { Total }\end{array}$} & -- & -- & -- & - & - \\
\hline & $3,723,361$ & $1,386,503$ & $, 647,048$ & 518,076 & 171,734 \\
\hline All species & $6,142,873$ & $2,960,959$ & $2,445,237$ & 562,927 & 173,750 \\
\hline
\end{tabular}


Table 25.--Number of short-log trees on commercial forest land by species group and diameter class, Minnesota, 1977

(In thousand trees)

\begin{tabular}{|c|c|c|c|c|c|c|c|c|c|c|c|}
\hline \multirow{3}{*}{ Species } & \multirow[b]{2}{*}{$\begin{array}{c}\text { All } \\
\text { classes }\end{array}$} & \multicolumn{10}{|c|}{ Diameter class (inches at breast height) } \\
\hline & & $\begin{array}{l}9.0- \\
10.9\end{array}$ & $\begin{array}{l}11.0- \\
12.9\end{array}$ & $\begin{array}{l}13.0- \\
14.9\end{array}$ & $\begin{array}{l}15.0- \\
16.9\end{array}$ & $\begin{array}{l}17.0- \\
18.9\end{array}$ & $\begin{array}{l}19.0- \\
20.9\end{array}$ & $\begin{array}{l}21.0- \\
22.9\end{array}$ & $\begin{array}{l}23.0- \\
28.9\end{array}$ & $\begin{array}{l}29.0- \\
38.9\end{array}$ & $39.0+$ \\
\hline & & & & & & & & & & & \\
\hline White pine & 93 & 37 & 8 & 17 & 20 & 3 & 2 & -- & 5 & 1 & -- \\
\hline Red pine & 63 & 31 & 18 & 9 & -- & 3 & 2 & -- & -- & -- & -- \\
\hline Jack pine & 417 & 239 & 115 & 49 & 11 & 3 & -- & -- & -- & -- & -- \\
\hline White spruce & 35 & 26 & 5 & -- & 4 & -- & -- & -- & -- & -- & -- \\
\hline Black spruce & 142 & 130 & 8 & -- & 4 & -- & -- & -- & -- & -- & -- \\
\hline Balsam fir & 427 & 342 & 49 & 10 & 12 & 14 & -- & -- & -- & -- & -- \\
\hline Tamarack & 268 & 175 & 81 & 6 & 3 & 3 & -- & -- & -- & -- & -- \\
\hline Northern white-cedar & 1,880 & 883 & 613 & 210 & 70 & 84 & 14 & 4 & 2 & - & -- \\
\hline Other softwoods & 59 & 52 & -- & -- & 4 & -- & 3 & -- & $\ldots$ & -- & -- \\
\hline Total & 3,384 & 1,915 & 897 & 301 & 128 & 110 & 21 & 4 & 7 & 1 & -- \\
\hline HARDWOODS: & & & & & & & & & & & \\
\hline Select white oak & 1,133 & -- & 492 & 208 & 215 & 89 & 51 & 42 & 29 & 7 & -- \\
\hline Select red oak & 864 & -- & 323 & 266 & 121 & 76 & 26 & 31 & 19 & 2 & -- \\
\hline other red oak & 25 & -- & 5 & 12 & -- & 3 & 3 & 2 & -- & -- & -- \\
\hline Hickory & 17 & -- & 5 & 10 & -- & -- & 2 & -- & -- & -- & -- \\
\hline Yellow birch & 90 & -- & 18 & 42 & 5 & 10 & -- & 2 & 13 & -- & -- \\
\hline Hard maple & 547 & -- & 174 & 184 & 96 & 29 & 33 & 6 & 24 & 1 & -- \\
\hline Soft maple & 160 & -- & 27 & 58 & 30 & 15 & 13 & 4 & 7 & 5 & 1 \\
\hline Ash & 347 & -- & 217 & 75 & 39 & 9 & 2 & 2 & 2 & 1 & -- \\
\hline Bal sam poplar & 455 & -- & 211 & 76 & 16 & 103 & 26 & 19 & 3 & -- & 1 \\
\hline Paper birch & 1,398 & -- & 595 & 278 & 307 & 131 & 35 & 8 & 44 & -- & -- \\
\hline Bigtooth aspen & 184 & -- & 85 & 57 & 19 & 23 & -- & -- & -- & -- & -- \\
\hline Quaking aspen & 3,909 & -- & 2,131 & 939 & 497 & 222 & 99 & 10 & 10 & 1 & -- \\
\hline Basswood & 384 & -- & 175 & 86 & 54 & 22 & 14 & 7 & 24 & 2 & -- \\
\hline Elm & 1,099 & -- & 510 & 212 & 153 & 84 & 54 & 33 & 38 & 12 & 3 \\
\hline Select hardwoods & 49 & -- & 7 & 5 & 24 & 10 & -- & 2 & 1 & -- & -- \\
\hline Other hardwoods & 310 & -- & 161 & 88 & 31 & 14 & 5 & 2 & 6 & 1 & 2 \\
\hline Noncommercial species & -- & -- & -- & -- & -- & -- & -- & -- & -- & -- & $=-$ \\
\hline Total & 10,971 & $=$ & 5,136 & 2,596 & 1,607 & 840 & 363 & 170 & 220 & 32 & 7 \\
\hline All species & 14,355 & 1,915 & 6,033 & 2,897 & 1,735 & 950 & 384 & 174 & 227 & 33 & 7 \\
\hline
\end{tabular}


Table 26.--Net volume of growing stock and sawtimber on commercial forest land by species group, Minnesota, $1 / 1962$ and 1977

\begin{tabular}{|c|c|c|c|c|}
\hline \multirow[b]{2}{*}{ Species } & \multicolumn{2}{|c|}{ Growing stock } & \multicolumn{2}{|c|}{ Sawtimber } \\
\hline & $1 / 1962$ & 1977 & $1 / 1962$ & 1977 \\
\hline & \multicolumn{2}{|c|}{ Thousand cubic feet } & \multicolumn{2}{|c|}{ 2/Thousand board feet } \\
\hline \multicolumn{5}{|l|}{ SOFTWOODS: } \\
\hline White pine & 191,796 & 194,797 & 900,060 & $1,068,372$ \\
\hline Red pine & 325,301 & 434,556 & $1,530,249$ & $1,952,896$ \\
\hline Jack pine & 733,320 & 593,704 & $1,402,842$ & $1,566,444$ \\
\hline Spruce & 813,347 & 717,920 & 849,704 & $1,109,131$ \\
\hline Balsam fir & 765,270 & 893,608 & 791,442 & $1,338,249$ \\
\hline Tamarack & 302,558 & 250,589 & 281,500 & 346,508 \\
\hline Northern white-cedar & 251,867 & 387,215 & 376,843 & $1,142,761$ \\
\hline Other softwoods & & 4,618 & -- & 6,228 \\
\hline Total & $3,383,459$ & $3,477,007$ & $6,132,640$ & $8,530,589$ \\
\hline \multicolumn{5}{|l|}{ HAROWOODS: } \\
\hline White oak & 278,877 & 358,272 & 817,696 & $1,076,044$ \\
\hline Red oak & 433,424 & 581,563 & $1,027,215$ & $1,820,036$ \\
\hline Yellow birch & 13,904 & 10,611 & 63,839 & 39,926 \\
\hline Hard maple & 134,865 & 189,024 & 455,532 & 489,619 \\
\hline Soft maple & 94,429 & 131,845 & 232,890 & 281,999 \\
\hline Ash & 426,107 & 538,086 & 758,002 & 993,952 \\
\hline Paper birch & 841,822 & $1,273,982$ & 491,034 & $1,234,388$ \\
\hline Aspen & $2,790,752$ & $3,410,720$ & $2,208,044$ & $5,974,671$ \\
\hline Basswood & 239,667 & 402,424 & 719,073 & $1,116,835$ \\
\hline Elm & 341,740 & 431,437 & $1,257,911$ & $1,607,307$ \\
\hline Other hardwoods & 464,614 & 649,026 & 710,952 & $1,441,832$ \\
\hline Total & $6,060,201$ & $7,976,990$ & $8,742,188$ & $16,076,609$ \\
\hline All species & $9,443,660$ & $11,453,997$ & $14,874,828$ & $24,607,198$ \\
\hline
\end{tabular}

$1 /$ Figures have been adjusted from those published after the 1962 survey to conform to $1977^{7}$ volumes because of changes in survey definitions and procedures.

2/International 1/4-inch rule. 


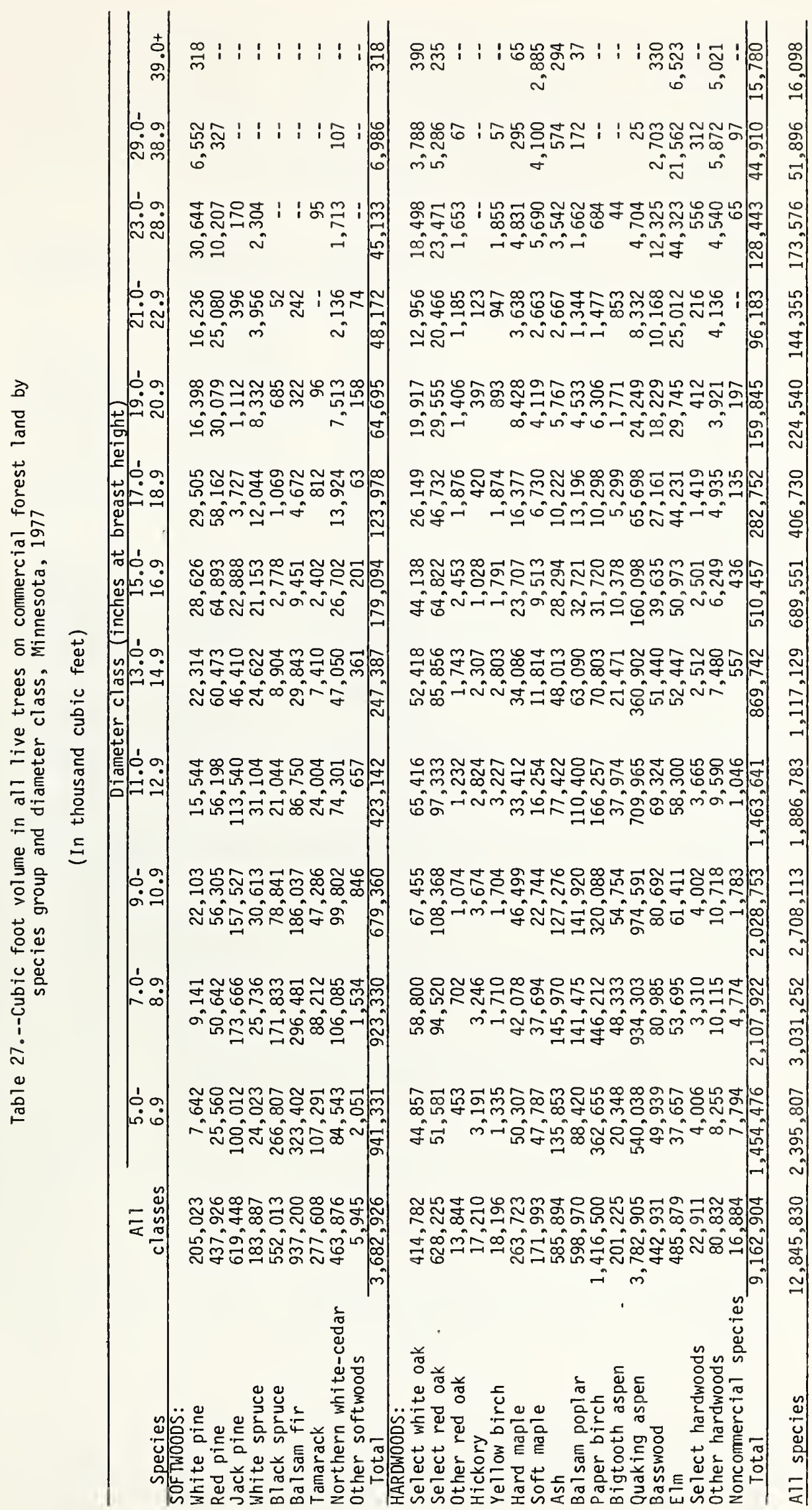


Table 28.--Net volume of timber on commercial forest land by class of timber and softwoods and hardwoods, Minnesota, 1977

(In thousand cubic feet)

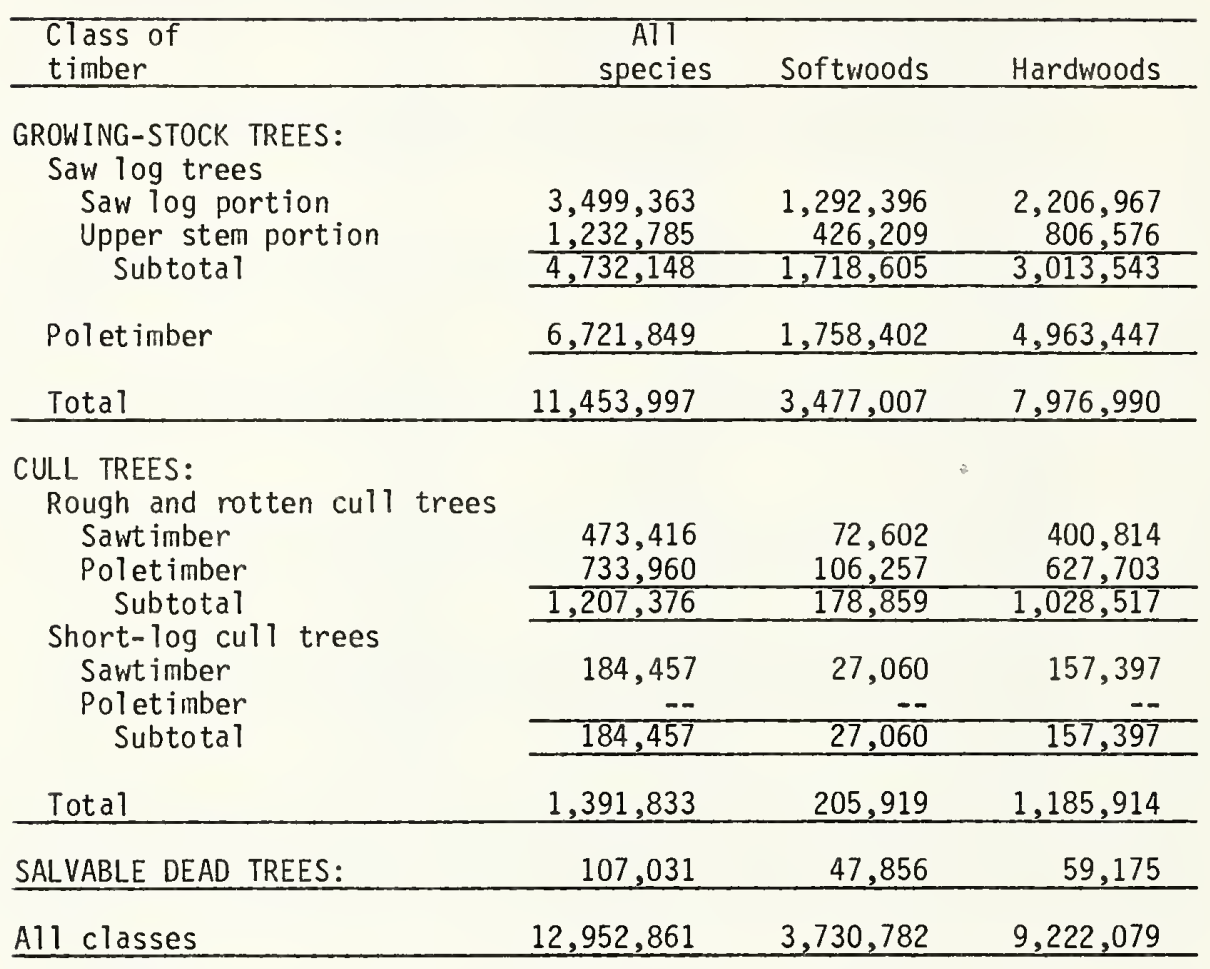




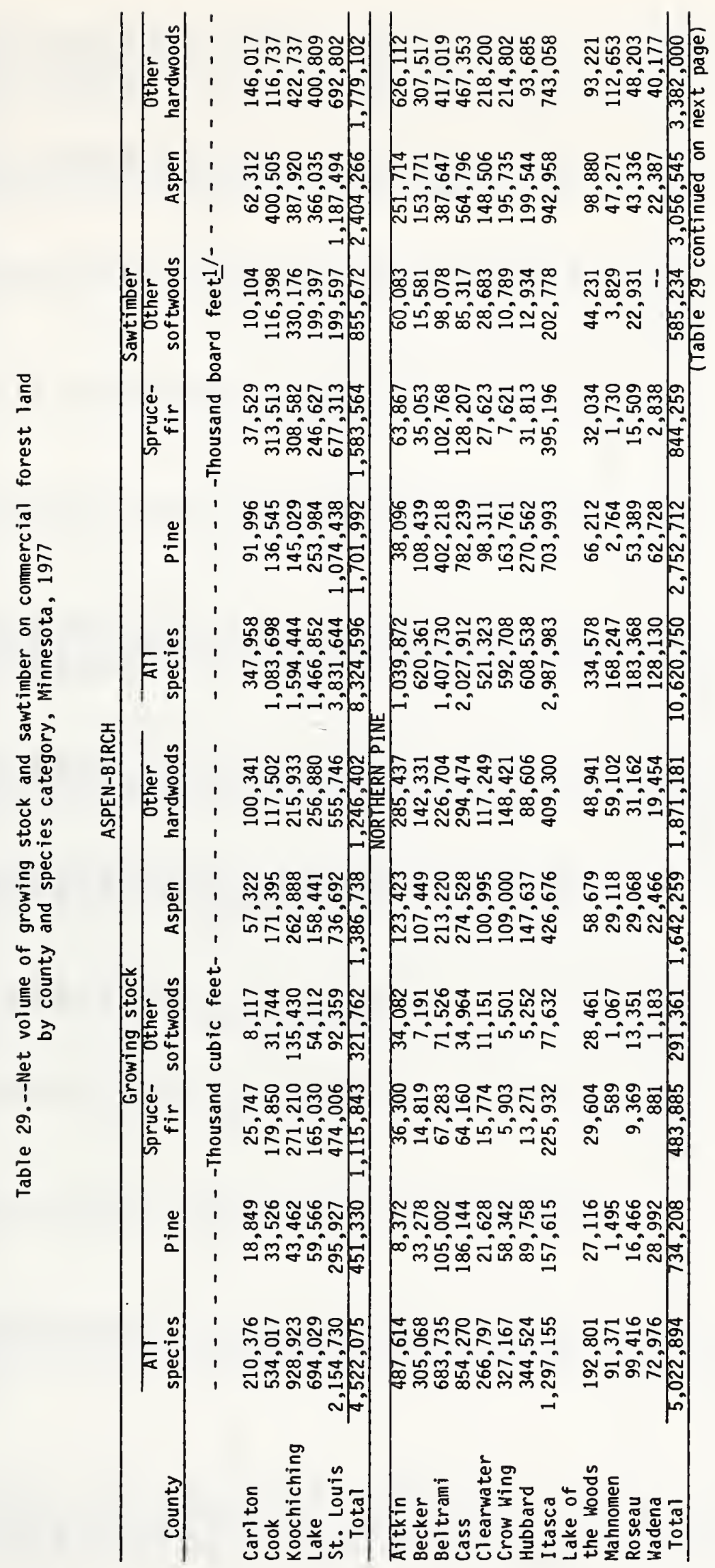




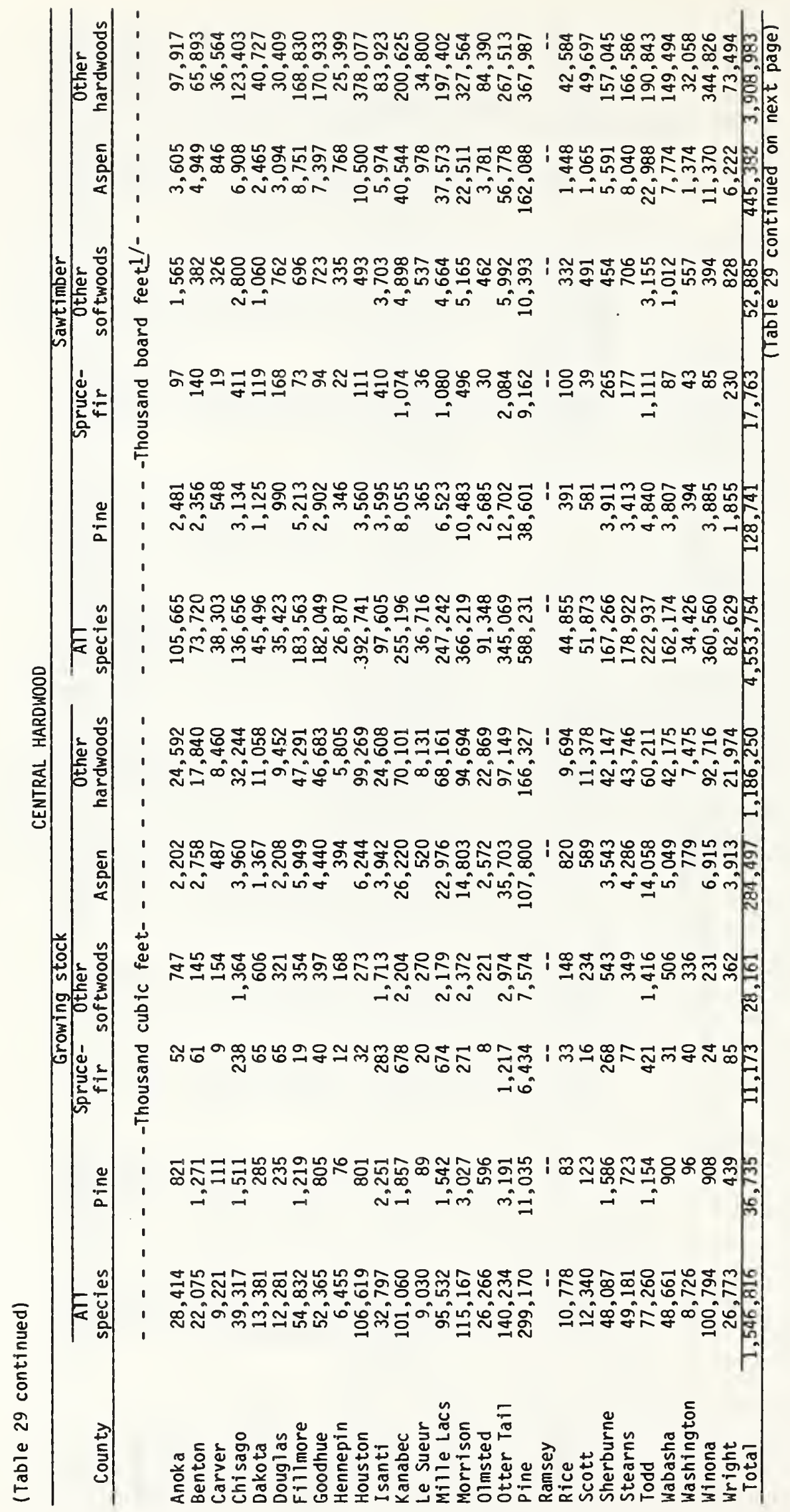




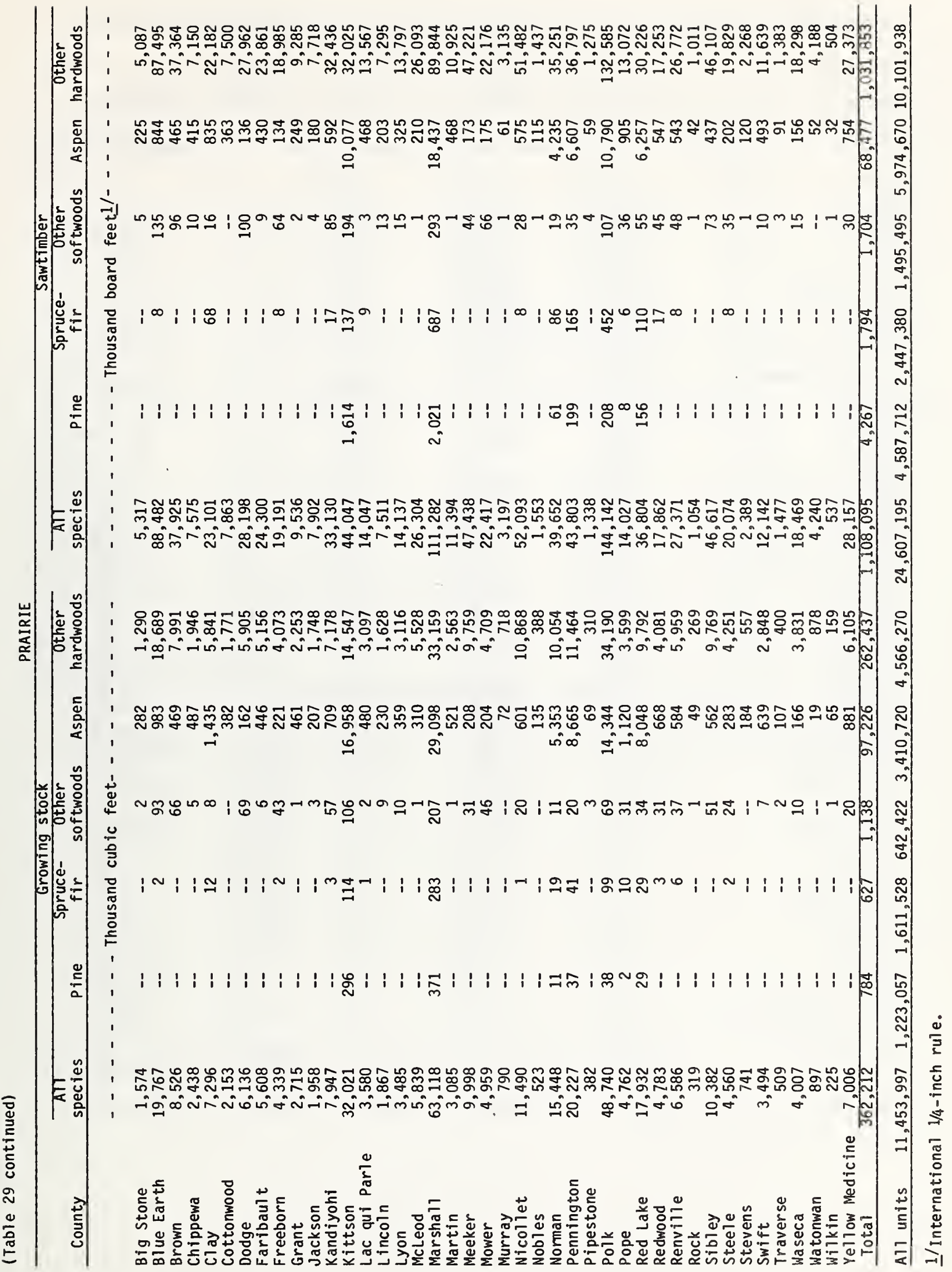


Table 30.--Net volume of growing stock, sawtimber, short-log, and rough and rotten trees on commercial forest land by individual species, Minnesota, 1977

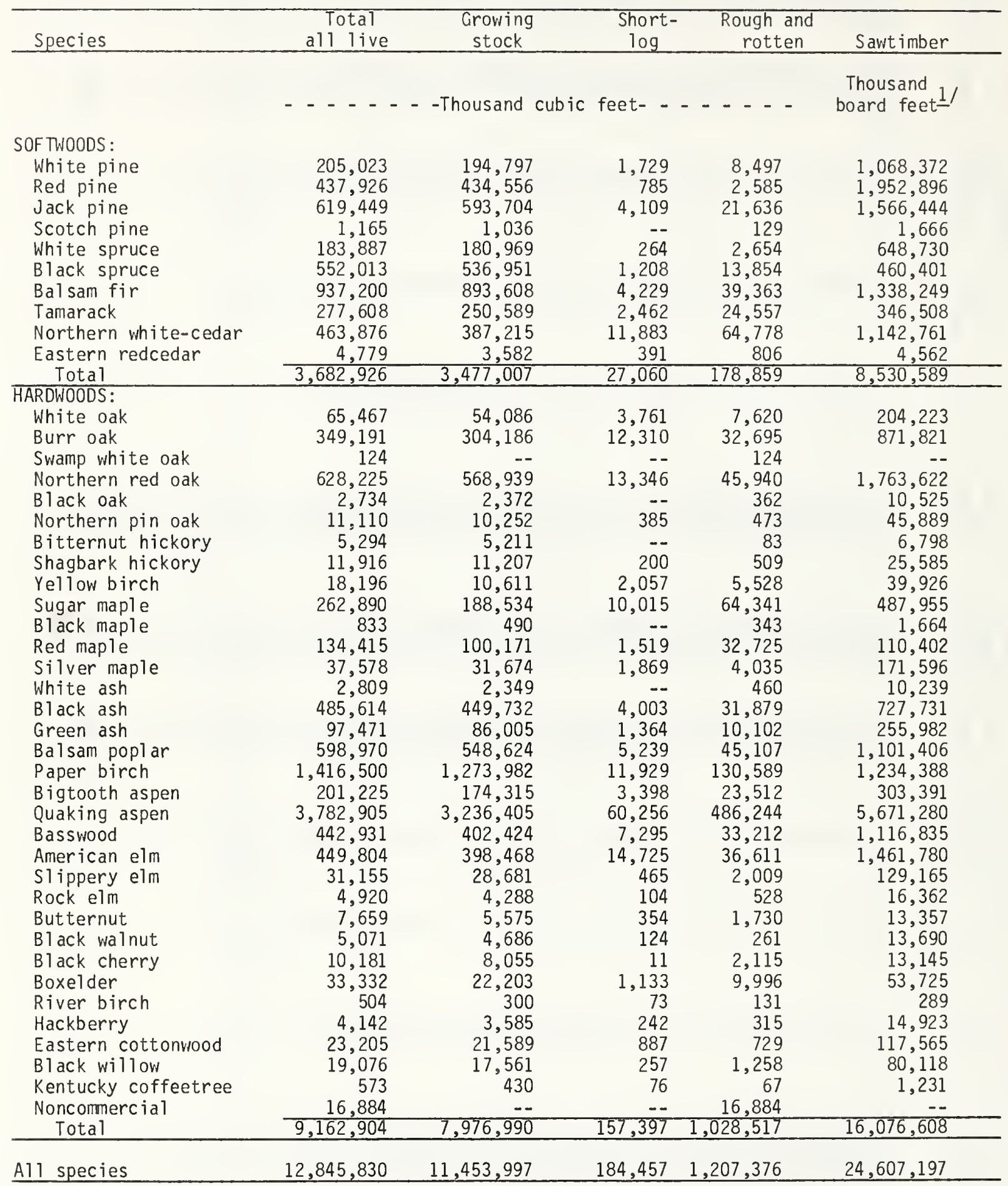

$1 /$ International $1 / 4$-inch rule. 
Table 31.--Net volume of growing stock on commercial forest 1 and by species group and Forest Survey Unit, Minnesota, 1977

(In thousand cubic feet)

\begin{tabular}{|c|c|c|c|c|c|}
\hline Species & $\begin{array}{c}\text { A11 } \\
\text { units }\end{array}$ & $\begin{array}{l}\text { Aspen- } \\
\text { Birch }\end{array}$ & $\begin{array}{c}\text { Northern } \\
\text { Pine }\end{array}$ & $\begin{array}{l}\text { Central } \\
\text { Hardwood }\end{array}$ & Prairie \\
\hline \multicolumn{6}{|l|}{ SOFTWOODS: } \\
\hline White pine & 194,797 & 91,561 & 90,495 & 12,741 & -- \\
\hline Red pine & 434,556 & 147,197 & 273,024 & 13,843 & 492 \\
\hline Jack pine & 593,704 & 212,572 & 370,689 & 10,151 & 292 \\
\hline White spruce & 180,969 & 136,485 & 42,389 & 1,705 & 390 \\
\hline Black spruce & 536,951 & 419,971 & 113,362 & 3,555 & 63 \\
\hline Bal sam fir & 893,608 & 559,387 & 328,134 & 5,913 & 174 \\
\hline Tamarack & 250,589 & 75,063 & 150,313 & 24,900 & 313 \\
\hline Northern white-cedar & 387,215 & 246,561 & 140,654 & & -- \\
\hline Other softwoods & 4,618 & 138 & 394 & 3,261 & 825 \\
\hline Total & $3,477,007$ & $1,888,935$ & $1,509,454$ & 76,069 & 2,549 \\
\hline \multicolumn{6}{|l|}{ HARDWOODS: } \\
\hline Select white oak & 358,272 & 5,277 & 119,986 & 172,960 & 60,049 \\
\hline Select red oak & 568,939 & 9,347 & 217,524 & 326,516 & 15,552 \\
\hline other red oak & 12,624 & -- & 1,015 & 11,087 & 522 \\
\hline Hickory & 16,418 & -- & 520 & 15,522 & 376 \\
\hline Yellow birch & 10,611 & 6,861 & 3,552 & 198 & -- \\
\hline Hard maple & 189,024 & 43,352 & 83,680 & 54,508 & 7,484 \\
\hline Soft maple & 131,845 & 38,495 & 49,757 & 40,258 & 3,335 \\
\hline Ash & 538,086 & 196,644 & 241,200 & 76,509 & 23,733 \\
\hline Balsam poplar & 548,624 & 244,477 & 265,529 & 12,839 & 25,779 \\
\hline Paper birch & $1,273,982$ & 620,775 & 538,764 & 111,395 & 3,048 \\
\hline Bigtooth aspen & 174,315 & 21,001 & 123,016 & 30,271 & 27 \\
\hline Quaking aspen & $3,236,405$ & $1,365,737$ & $1,519,243$ & 254,226 & 97,199 \\
\hline Basswood & 402,424 & 35,262 & 203,813 & 133,839 & 29,510 \\
\hline Elm & 431,437 & 45,431 & 141,808 & 185,338 & 58,860 \\
\hline Select hardwoods & 18,316 & 53 & 2,005 & 14,303 & 1,955 \\
\hline Other hardwoods & 65,668 & 428 & 2,028 & 30,978 & 32,234 \\
\hline Noncommercial species & -- & -- & -- & -. & -- \\
\hline Total & $7,976,990$ & $2,633,140$ & $3,513,440$ & $1,470,747$ & 359,663 \\
\hline All species & $11,453,997$ & $4,522,075$ & $5,022,894$ & $1,546,816$ & 362,212 \\
\hline
\end{tabular}


Table 32.--Net volume of sawtimber on commercial forest land by species group and Forest Survey Unit, Minnesota, 1977

(In thousand board feet) l/

\begin{tabular}{|c|c|c|c|c|c|}
\hline Species & $\begin{array}{c}\text { A11 } \\
\text { units }\end{array}$ & $\begin{array}{l}\text { Aspen- } \\
\text { Birch }\end{array}$ & $\begin{array}{l}\text { Northern } \\
\text { Pine }\end{array}$ & $\begin{array}{l}\text { Centrat } \\
\text { Hardwood }\end{array}$ & Prairie \\
\hline \multicolumn{6}{|l|}{ SOFTWOODS: } \\
\hline White pine & $1,068,372$ & 515,487 & 489,804 & 63,081 & -- \\
\hline Red pine & $1,952,896$ & 596,918 & $1,310,295$ & 42,516 & 3,167 \\
\hline Jack pine & $1,566,444$ & 589,587 & 952,613 & 23,144 & 1,100 \\
\hline White spruce & 648,730 & 471,316 & 168,448 & 7,172 & 1,794 \\
\hline Black spruce & 460,401 & 371,239 & 87,515 & 1,647 & - \\
\hline Balsam fir & $1,338,249$ & 741,009 & 588,296 & 8,944 & -- \\
\hline Tamarack & 346,508 & 86,831 & 210,950 & 48,280 & 447 \\
\hline Northern white-cedar & $1,142,761$ & 768,841 & 373,920 & & -- \\
\hline 0ther softwoods & 6,228 & -- & 364 & 4,605 & 1,259 \\
\hline Total & $8,530,589$ & $4,141,228$ & $4,182,205$ & 199,389 & 7,767 \\
\hline \multicolumn{6}{|l|}{ HARDWO0DS: } \\
\hline Select white oak & $1,076,044$ & 13,774 & 264,498 & 570,892 & 226,880 \\
\hline Select red oak & $1,763,622$ & 23,285 & 439,583 & $1,237,647$ & 63,107 \\
\hline Other red oak & 56,414 & -- & 1,141 & 52,870 & 2,403 \\
\hline Hickory & 32,383 & -- & 444 & 31,088 & 851 \\
\hline Yellow birch & 39,926 & 27,091 & 11,866 & 969 & -- \\
\hline Hard maple & 489,619 & 56,909 & 204,393 & 198,945 & 29,372 \\
\hline Soft maple & 281,998 & 30,931 & 75,450 & 158,376 & 17,241 \\
\hline Ash & 993,952 & 312,399 & 352,993 & 227,791 & 100,769 \\
\hline Bal sam poplar & $1,101,406$ & 481,135 & 572,553 & 18,664 & 29,054 \\
\hline Paper birch & $1,234,388$ & 591,221 & 570,799 & 69,492 & 2,876 \\
\hline Bigtooth aspen & 303,391 & 32,683 & 213,453 & 57,255 & -- \\
\hline Quaking aspen & $5,671,280$ & $2,371,583$ & $2,843,092$ & 388,128 & 68,477 \\
\hline Basswood & $1,116,835$ & 64,530 & 413,860 & 489,971 & 148,474 \\
\hline Elm & $1,607,307$ & 176,959 & 468,485 & 691,004 & 270,859 \\
\hline Select hardwoods & 40,192 & - & 2,223 & 32,630 & 5,339 \\
\hline Other hardwoods & 267,851 & 868 & 3,712 & 128,643 & 134,628 \\
\hline Noncommercial species & - & -- & - & - & -- \\
\hline Total & $16,076,608$ & $4,183,368$ & $6,438,545$ & $4,354,365$ & $1,100,330$ \\
\hline Al1 species & $24,607,197$ & $8,324,596$ & $10,620,750$ & $4,553,754$ & $1,108,097$ \\
\hline
\end{tabular}

1/International $1 / 4-i n c h$ rule. 


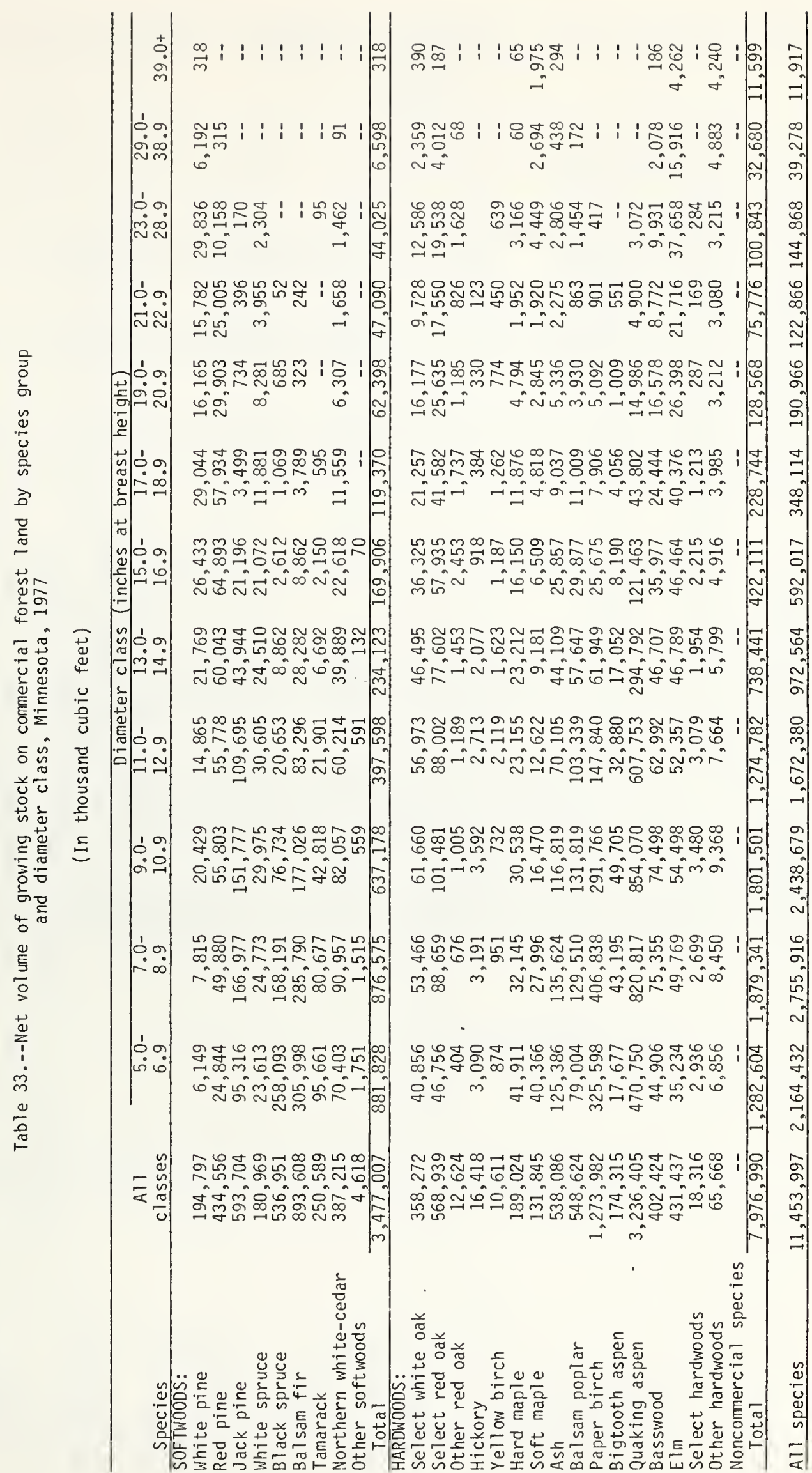




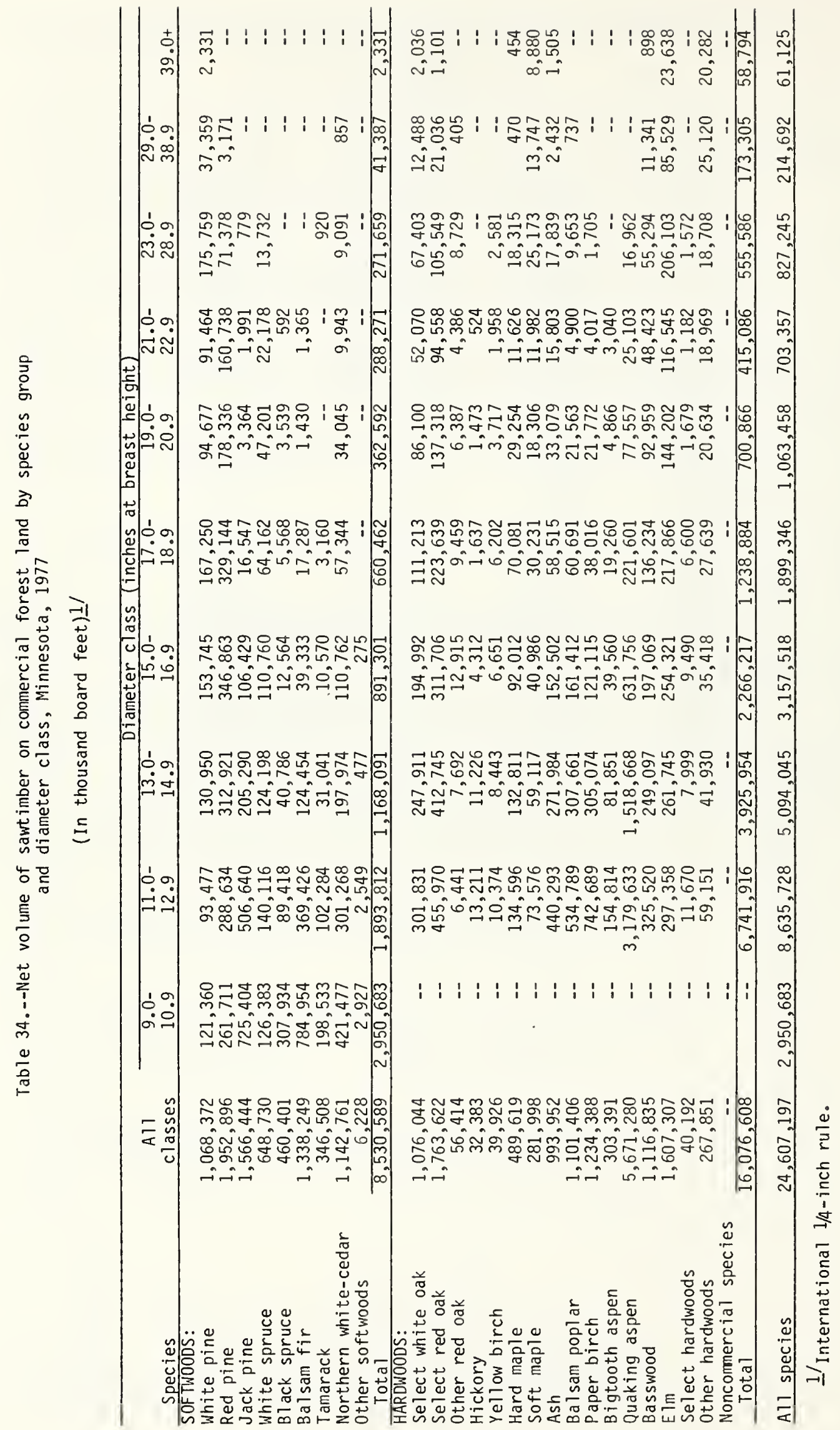


Table 35.--Net volume of growing stock on commercial forest 1 and by species group and forest type, Minnesota, 1977

(In thousand cubic feet)

\begin{tabular}{|c|c|c|c|c|c|c|c|c|}
\hline \multirow[b]{2}{*}{ Species } & \multirow[b]{2}{*}{$\begin{array}{c}\text { All } \\
\text { types }\end{array}$} & \multirow[b]{2}{*}{$\begin{array}{l}\text { Jack } \\
\text { pine }\end{array}$} & \multicolumn{5}{|c|}{ Forest type } & \multirow[b]{2}{*}{$\begin{array}{c}\text { Northern } \\
\text { white- } \\
\text { cedar }\end{array}$} \\
\hline & & & $\begin{array}{l}\text { Red } \\
\text { pine }\end{array}$ & $\begin{array}{l}\text { White } \\
\text { pine }\end{array}$ & $\begin{array}{c}\text { Bal sam } \\
\text { fir }\end{array}$ & $\begin{array}{l}\text { White } \\
\text { spruce }\end{array}$ & $\begin{array}{l}\text { Bl ack } \\
\text { spruce }\end{array}$ & \\
\hline SOFTWOODS: & & & & & & & & \\
\hline White pine & 194,797 & 2,788 & 45,377 & 52,529 & 11,887 & 1,009 & 2,787 & 2,631 \\
\hline Red pine & 434,556 & 38,735 & 251,343 & 16,619 & 12,406 & 1,119 & 1,839 & 3,477 \\
\hline Jack pine & 593,704 & 435,520 & 16,837 & 5,630 & 6,558 & 2,506 & 15,145 & 1,862 \\
\hline White spruce & 180,969 & 2,337 & 4,222 & 6,694 & 34,120 & 20,094 & 8,614 & 2,363 \\
\hline Black spruce & 536,951 & 16,129 & 1,403 & 803 & 54,748 & 1,244 & 342,917 & 38,975 \\
\hline Balsam fir & 893,608 & 5,764 & 14,921 & 3,850 & 299,047 & 4,765 & 29,742 & 33,255 \\
\hline Tamarack & 250,589 & 125 & 281 & -- & 11,015 & 624 & 37,677 & 11,725 \\
\hline Northern & & & & & & & & \\
\hline whi te-cedar & 387,215 & -- & 312 & 211 & 42,370 & 1,760 & 10,264 & 235,540 \\
\hline 0 ther so f twoods & 4,618 & -- & 589 & -- & -- & -- & 184 & -- \\
\hline Total & $3,477,007$ & 501,398 & 335,285 & 86,336 & 472,151 & 33,121 & 449,169 & 329,828 \\
\hline \multicolumn{9}{|l|}{ HARDWOODS: } \\
\hline Select white oak & 358,272 & 854 & 226 & 623 & 613 & 29 & -- & -- \\
\hline Select red oak & 568,939 & 2,628 & 1,037 & 571 & 152 & -- & 197 & -- \\
\hline other red oak & 12,624 & -- & -- & -- & -- & -- & -- & -- \\
\hline Hickory & 16,418 & -- & -- & -- & -- & -- & -- & -- \\
\hline Yellow birch & 10,611 & -- & -- & -- & 308 & -- & -- & 715 \\
\hline Hard maple & 189,024 & -- & -- & 221 & 607 & -- & -- & - \\
\hline Soft maple & 131,845 & 1,334 & 56 & 870 & 2,426 & -- & 286 & 57 \\
\hline Ash & 538,086 & & 129 & 345 & 8,550 & -- & 2,115 & 9,017 \\
\hline Balsam poplar & 548,624 & 579 & 559 & 46 & 19,671 & 722 & 1,110 & 6,635 \\
\hline Paper birch & $1,273,982$ & 11,385 & 24,693 & 4,421 & 93,342 & 8,572 & 7,902 & 24,318 \\
\hline Bigtooth aspen & 174,315 & 3,248 & 2,887 & 37 & 789 & -- & 311 & 516 \\
\hline Quaking aspen & $3,236,405$ & 38,439 & 14,328 & 10,143 & 77,556 & 7,516 & 32,326 & 7,309 \\
\hline Basswood & 402,424 & 63 & -- & 465 & 1,448 & -- & -- & -- \\
\hline Elm & 431,437 & 124 & 43 & -- & 1,530 & -- & 79 & 172 \\
\hline Select hardwoods & 18,316 & -- & -- & -- & -- & -- & -- & -- \\
\hline Other hardwoods & 65,668 & 225 & 118 & -- & -- & -- & -- & -- \\
\hline Nonc ommercial & & & & & & & & \\
\hline species & -- & -- & -- & -- & -- & -- & -- & -- \\
\hline Total & $7,976,990$ & 58,943 & 44,076 & 17,742 & 206,992 & 16,839 & 44,326 & 48,739 \\
\hline All species & $11,453,997$ & 560,341 & 379,361 & 104,078 & 679,143 & 49,960 & 493,495 & 378,567 \\
\hline
\end{tabular}




\begin{tabular}{|c|c|c|c|c|c|c|c|c|}
\hline Species & Tamarack & $\begin{array}{c}\text { Oak } \\
\text { hickory } \\
\end{array}$ & $\begin{array}{c}\text { Elm-ash- } \\
\text { cottonwood } \\
\end{array}$ & $\begin{array}{c}\text { Maple- } \\
\text { basswood }\end{array}$ & Aspen & $\begin{array}{l}\text { Paper } \\
\text { birch }\end{array}$ & $\begin{array}{l}\text { Bal sam } \\
\text { poplar }\end{array}$ & $\begin{array}{c}\text { Non- } \\
\text { stocked }\end{array}$ \\
\hline \multicolumn{9}{|l|}{ SOFTWOODS: } \\
\hline White pine & 1,248 & 2,422 & 1,583 & 12,990 & 43,050 & 14,053 & 443 & -- \\
\hline Red pine & 514 & 6,075 & 322 & 766 & 75,167 & 25,865 & 309 & -- \\
\hline Jack pine & 1,047 & 6,565 & 442 & 1,700 & 87,605 & 9,032 & 1,162 & 2,093 \\
\hline White spruce & 488 & 554 & 5,123 & 7,715 & 65,257 & 17,996 & 5,392 & \\
\hline Bl ack spruce & 21,379 & 86 & 2,265 & 975 & 48,382 & 4,671 & 2,537 & 437 \\
\hline Balsam fir & 3,478 & 577 & 35,475 & 42,678 & 292,578 & 84,111 & 39,590 & 3,777 \\
\hline Tamarack & 161,129 & 214 & 3,179 & 2,566 & 11,872 & 4,983 & 4,371 & 828 \\
\hline Northern & & & & & & & & \\
\hline whi te-cedar & 4,706 & -- & 29,393 & 12,048 & 31,292 & 12,495 & 6,419 & 405 \\
\hline Other softwoods & -- & 2,275 & & 1,019 & 45 & 296 & 121 & -- \\
\hline Total & 193,989 & 18,768 & 77,871 & 82,457 & 655,248 & 173,502 & 60,344 & 7,540 \\
\hline \multicolumn{9}{|l|}{ HARDWOODS: } \\
\hline Select white oak & -- & 186,573 & 7,989 & 64,565 & 85,978 & 8,692 & 1,924 & 206 \\
\hline Select red oak & 197 & 345,887 & 1,118 & 66,373 & 120,485 & 29,402 & 892 & -- \\
\hline other red oak & -. & 9,034 & 317 & 1,774 & 1,296 & 69 & 134 & -- \\
\hline Hickory & -- & 12,728 & -- & 2,863 & -- & 827 & -- & -- \\
\hline Yellow birch & -- & - & 1,814 & 6,110 & 250 & 1,185 & 229 & -- \\
\hline Hard maple & -- & 5,497 & 1,901 & 147,695 & 23,019 & 8,550 & 1,430 & 104 \\
\hline Soft maple & 253 & 5,064 & 28,823 & 34,196 & 40,287 & 17,832 & 361 & -- \\
\hline Ash & 424 & 9,393 & 267,189 & 95,747 & 98,957 & 24,632 & 20,901 & 623 \\
\hline Bal sam poplar & 2,308 & 1,949 & 21,942 & 16,858 & 231,042 & 23,691 & 221,373 & 139 \\
\hline Paper birch & 3,135 & 44,595 & 28,129 & 81,071 & 448,945 & 467,891 & 24,200 & 1,383 \\
\hline Bigtooth aspen & -- & 11,001 & 1,233 & 9,597 & 135,755 & 8,588 & 353 & -- \\
\hline Quaking aspen & 2,604 & 53,146 & 22,274 & 85,594 & $2,697,885$ & 124,144 & 59,467 & 3,674 \\
\hline Basswood & -- & 30,356 & 11,609 . & 271,204 & 69,857 & 15,372 & 2,050 & -- \\
\hline Elm & 910 & 36,307 & 70,521 & 225,013 & 67,648 & 14,916 & 13,654 & 520 \\
\hline Select hardwoods & -- & 10,080 & 607 & 5,204 & 1,911 & 389 & 67 & 58 \\
\hline other hardwoods & -- & 3,908 & 40,945 & 16,554 & 3,362 & -- & 213 & 343 \\
\hline $\begin{array}{c}\text { Noncommercial } \\
\text { species }\end{array}$ & & & -- & & -- & & & \\
\hline $\begin{array}{l}\text { species } \\
\text { Total }\end{array}$ & 9.831 & $\frac{--}{518}$ & बन & $0 .--$ & 667 & $746-\frac{--}{180}$ & 347248 & 050 \\
\hline & & & & & & & & \\
\hline All species & 203,820 & 784,286 & 584,282 & $1,212,875$ & $4,681,925$ & 919,682 & 407,592 & 14,590 \\
\hline
\end{tabular}


Table 36.--Net volume of sawtimber on commercial forest land by species group and forest type, Minnesota, 1977

(In thousand board feet) 1 /

\begin{tabular}{|c|c|c|c|c|c|c|c|c|}
\hline & & & & & Forest typ & & & \\
\hline Species & $\begin{array}{l}\text { A11 } \\
\text { types }\end{array}$ & $\begin{array}{l}\text { Jack } \\
\text { pine }\end{array}$ & $\begin{array}{l}\text { Red } \\
\text { pine }\end{array}$ & $\begin{array}{l}\text { White } \\
\text { pine }\end{array}$ & $\begin{array}{l}\text { Bal sam } \\
\text { fir }\end{array}$ & $\begin{array}{l}\text { White } \\
\text { spruce }\end{array}$ & $\begin{array}{l}\text { Bl ack } \\
\text { spruce }\end{array}$ & $\begin{array}{l}\text { Northern } \\
\text { white- } \\
\text { cedar }\end{array}$ \\
\hline $\begin{array}{l}\text { SOFWoods: } \\
\text { White pine } \\
\text { Red pine } \\
\text { Jack pine } \\
\text { White spruce } \\
\text { Black spruce } \\
\text { Balsam fir } \\
\text { Tamarack } \\
\text { Northern }\end{array}$ & $\begin{array}{r}1,068,372 \\
1,952,896 \\
1,566,444 \\
648,730 \\
460,401 \\
1,338,249 \\
346,508\end{array}$ & $\begin{array}{r}10,856 \\
135,809 \\
1,051,843 \\
4,381 \\
11,469 \\
3,897 \\
687\end{array}$ & $\begin{array}{r}237,978 \\
1,093,109 \\
45,843 \\
13,789 \\
998 \\
46,705 \\
354\end{array}$ & $\begin{array}{r}289,021 \\
88,319 \\
28,580 \\
29,458 \\
1,192 \\
7,691 \\
--\end{array}$ & $\begin{array}{r}72,837 \\
64,394 \\
25,931 \\
125,019 \\
79,000 \\
394,770 \\
25,465\end{array}$ & $\begin{array}{r}6,649 \\
4,588 \\
10,876 \\
65,686 \\
1,404 \\
5,506 \\
2,388\end{array}$ & $\begin{array}{r}16,097 \\
9,963 \\
43,528 \\
26,213 \\
180,844 \\
25,026 \\
40,026\end{array}$ & $\begin{array}{r}16,202 \\
18,696 \\
6,303 \\
8,914 \\
64,052 \\
29,443 \\
14,828\end{array}$ \\
\hline $\begin{array}{l}\text { whi te-cedar } \\
\text { other softwoods }\end{array}$ & $\begin{array}{r}1,142,761 \\
6,228\end{array}$ & -- & 1,101 & $\begin{array}{r}428 \\
--\end{array}$ & $\begin{array}{r}132,944 \\
\ldots\end{array}$ & 7,411 & 17,620 & $\begin{array}{r}619,635 \\
--\end{array}$ \\
\hline Tota 1 & $8,530,589$ & $1,218,942$ & $1,439,877$ & 444,689 & 920,360 & 104,508 & 359,317 & 778,073 \\
\hline HARDWOODS: & & & & & & & & \\
\hline Select white oak & $1,076,044$ & 393 & $\overline{-}$ & 552 & 1,904 & -- & -- & -- \\
\hline Select red oak & $1,763,622$ & 1,531 & 2,005 & 2,376 & 438 & -- & - & -- \\
\hline other red oak & 56,414 & -- & -- & -- & -- & -- & -- & -- \\
\hline Hickory & 32,383 & - & -- & -- & -- & -- & -- & -- \\
\hline Yellow birch & 39,926 & - & -- & -- & 646 & - & -- & 2,236 \\
\hline Hard maple & 489,619 & - & -- & 1,344 & -- & -- & $-\infty$ & $-\infty$ \\
\hline Soft maple & 281,998 & -- & -- & 346 & 7,623 & -- & 684 & -- \\
\hline Ash & 993,952 & -- & -- & 368 & 12,125 & -- & 6,719 & 16,218 \\
\hline Balsam poplar & $1,101,406$ & 975 & -- & -- & 30,680 & 750 & 1,048 & 16,036 \\
\hline Paper birch & $1,234,388$ & 3,909 & 36,621 & 3,857 & 141,043 & 11,322 & 4,826 & 42,718 \\
\hline Bigtooth aspen & 303,391 & 1,181 & 318 & -- & 2,475 & -- & 393 & 1,176 \\
\hline Quaking aspen & $5,671,280$ & 61,514 & 19,307 & 37,026 & 123,947 & 16,488 & 31,878 & 15,719 \\
\hline Basswood & $1,116,835$ & -- & -- & 1,296 & 4,582 & -- & - & -- \\
\hline Elm & $1,607,307$ & 424 & -- & - & 4,617 & -- & - & 762 \\
\hline Select hardwoods & 40,192 & -- & -- & -- & -- & -- & -- & -- \\
\hline $\begin{array}{l}\text { Other hardwoods } \\
\text { Noncommercial }\end{array}$ & 267,851 & -- & -- & -- & -- & -- & -- & -- \\
\hline species & -- & -- & -- & -- & -- & -- & -- & -- \\
\hline Total & $16,076,608$ & 69,927 & 58,251 & 47,165 & 330,080 & 28,560 & 45,548 & 94,865 \\
\hline Al1 species & $24,607,197$ & $1,288,869$ & $1,498,128$ & 491,854 & $1,250,440$ & 133,068 & 404,865 & 872,938 \\
\hline
\end{tabular}

1/International $1 / 4$-inch rule. 


\begin{tabular}{|c|c|c|c|c|c|c|c|c|}
\hline Species & Tamarack & $\begin{array}{c}\text { Oak } \\
\text { hickory }\end{array}$ & $\begin{array}{c}\text { Elm-ash- } \\
\text { cottonwood }\end{array}$ & $\begin{array}{c}\text { Maple- } \\
\text { basswood }\end{array}$ & Aspen & $\begin{array}{l}\text { Paper } \\
\text { birch }\end{array}$ & $\begin{array}{l}\text { Bal sam } \\
\text { poplar }\end{array}$ & $\begin{array}{c}\text { Non- } \\
\text { stocked }\end{array}$ \\
\hline \multicolumn{9}{|l|}{ SOFTWOODS: } \\
\hline $\begin{array}{l}\text { White pine } \\
\text { Red pine }\end{array}$ & $\begin{array}{l}1,454 \\
2,293\end{array}$ & $\begin{array}{l}13,968 \\
27,070\end{array}$ & $\begin{array}{r}10,261 \\
1,128\end{array}$ & $\begin{array}{r}73,792 \\
4353\end{array}$ & $\begin{array}{l}235,419 \\
365,963\end{array}$ & $\begin{array}{r}75,224 \\
135,609\end{array}$ & $\begin{array}{l}2,614 \\
1,602\end{array}$ & -- \\
\hline Jack pine & 2,655 & 18,743 & 1,214 & 7,218 & 284,213 & 32,953 & 896 & 5,648 \\
\hline White spruce & 1,028 & 2,357 & 19,458 & 34,788 & 217,387 & 79,613 & 20,639 & -- \\
\hline Black spruce & 12,464 & 353 & 4,429 & 1,720 & 84,154 & 10,270 & 7,073 & 979 \\
\hline Balsam fir & 4,453 & 493 & 59,563 & 94,096 & 459,104 & 125,661 & 71,838 & 10,003 \\
\hline Tamarack & 177,555 & 1,101 & 7,247 & 6,685 & 36,068 & 14,748 & 17,602 & 1,754 \\
\hline Nor thern & & & & & & & & \\
\hline white-cedar & 11,911 & 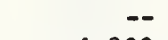 & 108,288 & 52,133 & 118,607 & 48,256 & 22,913 & 1,514 \\
\hline Other softwoods & & 4,039 & 364 & 1,825 & -- & -- & -- & -- \\
\hline Total & 219,813 & 68,124 & 211,952 & 276,610 & $1,800,915$ & 522,334 & 145,177 & 19,898 \\
\hline \multicolumn{9}{|l|}{ HARDWOODS: } \\
\hline Select white oak & -- & 601,375 & 29,402 & 237,231 & 181,871 & 17,761 & 4,879 & 676 \\
\hline Select red oak & 1,082 & $1,165,499$ & 926 & 247,767 & 273,100 & 65,337 & 3,561 & -- \\
\hline other red oak & -- & 42,719 & 1,718 & 9,021 & 2,231 & -- & 725 & -- \\
\hline Hickory & -- & 23,625 & -- & 5,456 & -- & 3,302 & -- & -- \\
\hline Yell ow birch & -- & & 7,306 & 24,162 & 463 & 4,218 & 895 & -- \\
\hline Hard maple & -- & 14,787 & 4,913 & 435,094 & 26,390 & 6,490 & 601 & -- \\
\hline Soft maple & -- & 9,080 & 148,521 & 79,370 & 31,582 & 4,792 & -- & -- \\
\hline Ash & -- & 20,987 & 467,143 & 278,329 & 127,516 & 31,683 & 30,240 & 2,624 \\
\hline Bal sam poplar & 4,939 & 4,350 & 64,585 & 49,547 & 449,184 & 52,074 & 427,238 & -- \\
\hline Paper birch & 2,416 & 29,524 & 44,761 & 145,752 & 358,611 & 370,387 & 37,849 & 792 \\
\hline Bigtooth aspen & -- & 29,064 & 2,877 & 29,072 & 219,687 & 17,148 & & -- \\
\hline Quaking aspen & 3,989 & 94,318 & 53,728 & 198,888 & $4,572,282$ & 307,130 & 128,498 & 6,568 \\
\hline Basswood & -- & 78,306 & 40,612 & 860,089 & 98,727 & 29,965 & 3,258 & -- \\
\hline Elm & 811 & 122,620 & 270,664 & 936,185 & 182,843 & 39,943 & 46,278 & 2,160 \\
\hline Select hardwoods & -- & 21,447 & 226 & 15,359 & 2,483 & 677 & -- & -- \\
\hline other hardwoods & -- & 12,123 & 177,158 & 68,153 & 8,492 & -- & -- & 1,925 \\
\hline \multicolumn{9}{|l|}{ Nonc ommercial } \\
\hline species & -- & -- & -- & -- & -- & -- & -- & -- \\
\hline Total & 13,231 & $2,269,824$ & $1,314,540$ & $3,619,475$ & $6,535,462$ & 950,907 & 684,022 & 14,745 \\
\hline 11 species & 233,050 & $2,337,948$ & $1,526,492$ & $3,896,085$ & $8,336,377$ & $1,473,241$ & 829,199 & 34,643 \\
\hline
\end{tabular}




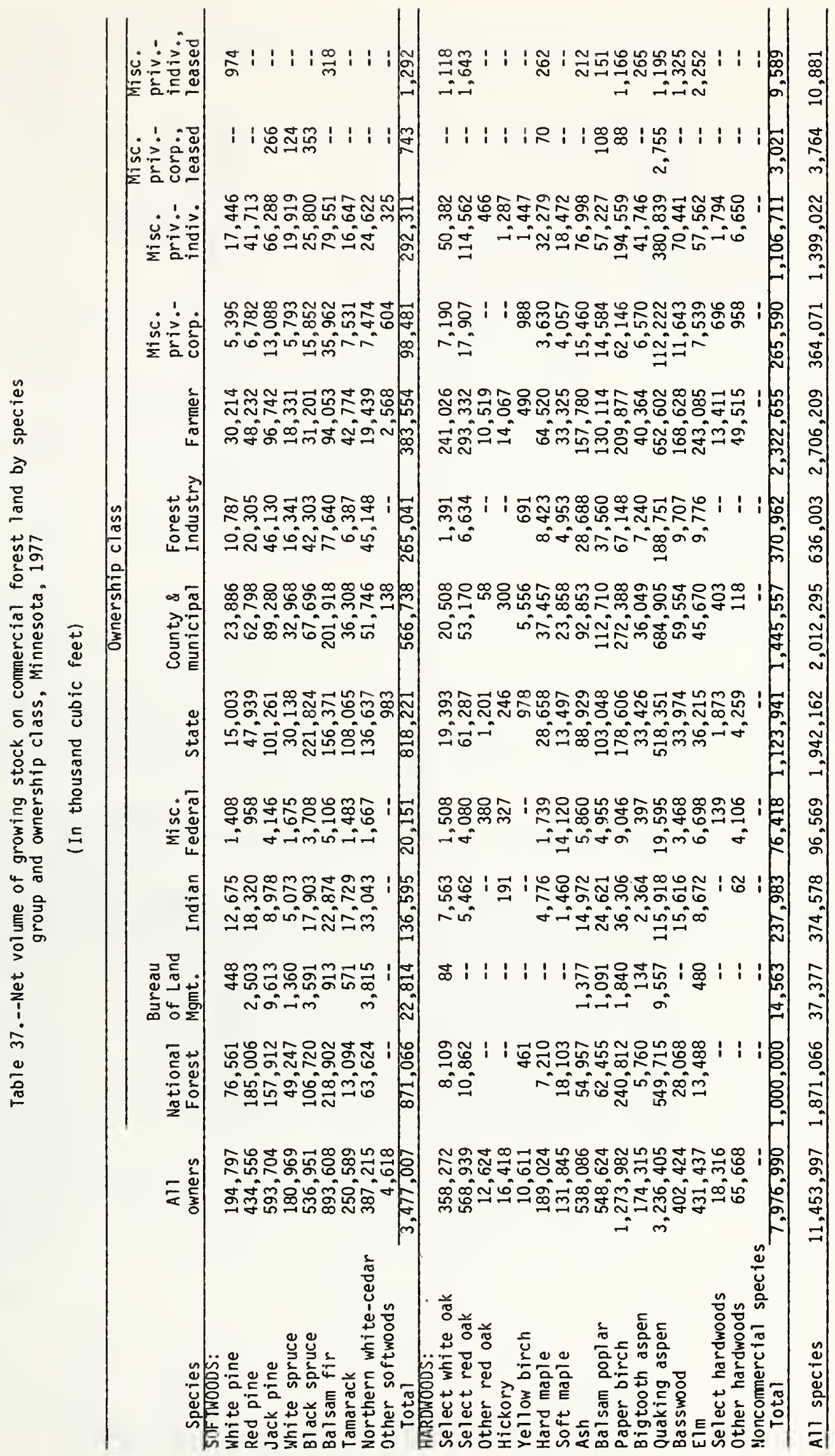




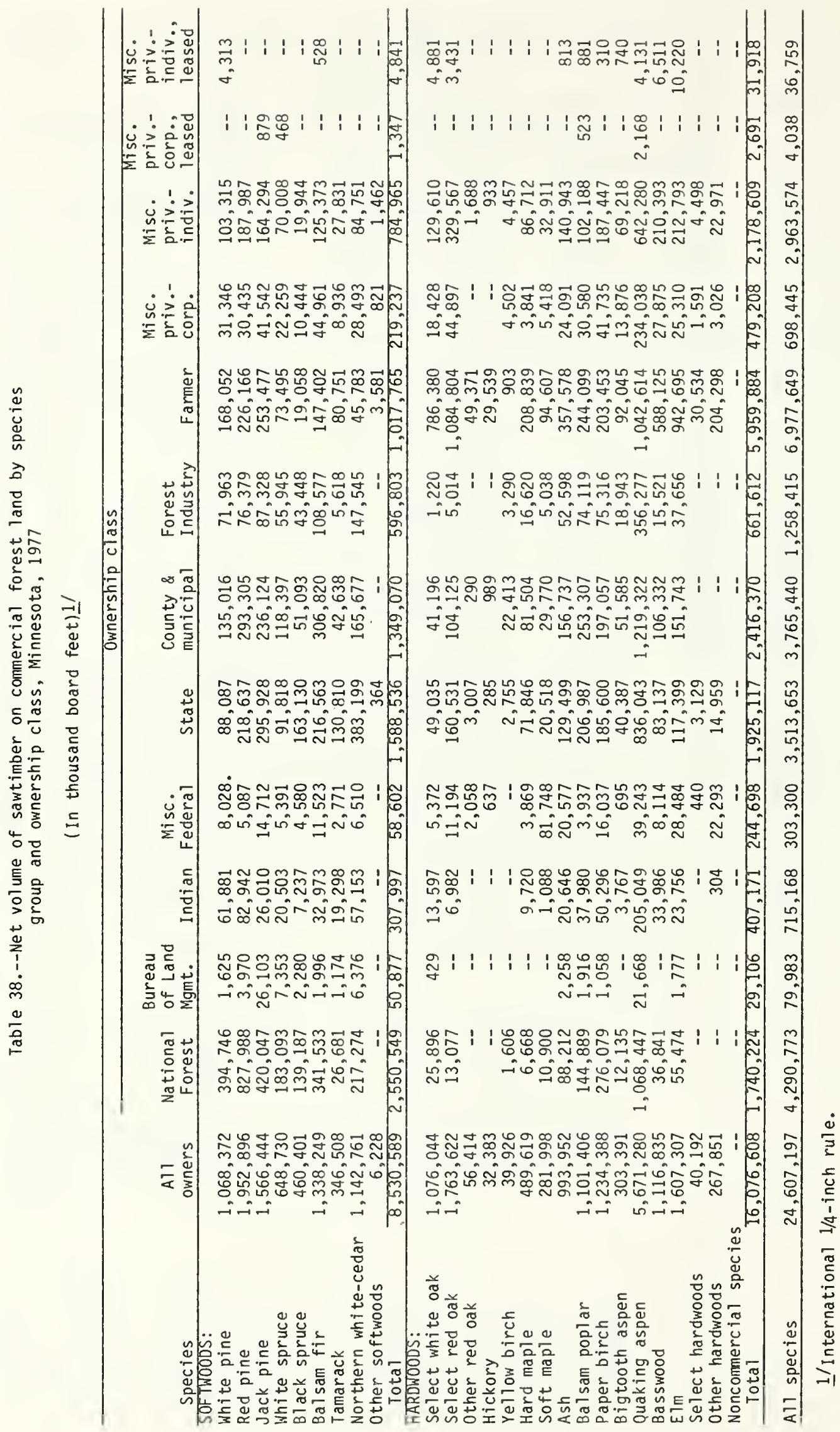


Table 39.--Net volume of growing stock on commercial forest land by forest type and stand-age class, Minnesota, 1977

(In thousand cubic feet)

\begin{tabular}{|c|c|c|c|c|c|c|c|}
\hline \multirow{2}{*}{$\begin{array}{l}\text { Forest } \\
\text { type }\end{array}$} & \multirow{2}{*}{$\begin{array}{c}\text { All } \\
\text { classes } \\
\end{array}$} & \multicolumn{6}{|c|}{ Stand-age class (years) } \\
\hline & & $0-10$ & $11-20$ & $21-30$ & $31-40$ & $41-50$ & $51-60$ \\
\hline Jack pine & 560,341 & 4,681 & 3,194 & 24,517 & 115,786 & 143,135 & 147,803 \\
\hline Red pine & 379,361 & 2,491 & 6,730 & 8,577 & 40,312 & 32,630 & 33,291 \\
\hline White pine & 104,078 & & 491 & & 1,329 & 10,419 & 7,950 \\
\hline Balsam fir & 679,143 & 12,674 & 18,644 & 31,363 & 65,442 & 211,002 & 193,493 \\
\hline White spruce & 49,960 & 304 & 384 & 1,721 & 4,189 & 15,454 & 11,761 \\
\hline Black spruce & 493,495 & 3,624 & 17,395 & 32,662 & 39,987 & 61,332 & 101,045 \\
\hline Northern white-cedar & 378,567 & 551 & 1,833 & 5,010 & 10,351 & 10,892 & 25,634 \\
\hline Tamarack & 203,820 & 3,117 & 13,425 & 9,423 & 7,848 & 20,980 & 40,830 \\
\hline 0ak-hickory & 784.286 & 4,577 & 9,899 & 2,910 & 53,113 & 136,848 & 133,132 \\
\hline Elm-ash-cottonwood & 584,282 & 9,677 & 14,165 & 14,278 & 18,361 & 67,374 & 102,512 \\
\hline Maple-basswood & 212,875 & 8,049 & 14,472 & 15,438 & 80,412 & 173,660 & 234,623 \\
\hline Aspen & 681,925 & 159,232 & 149,722 & 250,317 & 816,553 & $1,443,009$ & $1,151,136$ \\
\hline Paper birch & 919,682 & 5,063 & 14,592 & 16,703 & 95,456 & 271,439 & 240,579 \\
\hline Baisam poplar & & 13,990 & 15,138 & 18,044 & 64,316 & 131,327 & 79,004 \\
\hline Nonstocked & & & 151 & - & 106 & 348 & - \\
\hline All types & $11,453,997$ & 241,916 & 280,235 & 430,963 & $1,413,561$ & $2,729,849$ & $2,502,793$ \\
\hline
\end{tabular}

(Table 39 continued)

\begin{tabular}{|c|c|c|c|c|c|c|c|}
\hline \multirow{2}{*}{$\begin{array}{l}\text { Forest } \\
\text { type }\end{array}$} & \multicolumn{7}{|c|}{ Stand-age class (years) } \\
\hline & $61-70$ & $71-80$ & $81-90$ & $91-100$ & $101-120$ & $121-140$ & $141+$ \\
\hline Jack pine & 87,204 & 9,132 & 12,811 & 9,533 & 2,545 & -- & -- \\
\hline Red pine & 19,727 & 138,196 & 32,424 & 62,010 & 2,973 & -- & -- \\
\hline White pine & 14,846 & 23,017 & 4,335 & 27,989 & 13,702 & -- & -- \\
\hline Balsam fir & 88,787 & 29,075 & 11,850 & 13,682 & 3,131 & -- & -- \\
\hline White spruce & 4,956 & 1,530 & 3,496 & 5,173 & 992 & -- & -- \\
\hline Black spruce & 58,575 & 73,907 & 45,927 & 37,959 & 8,520 & 12,562 & -- \\
\hline Northern white-cedar & 47,916 & 33,323 & 55,880 & 53,773 & 46,602 & 84,195 & 2,607 \\
\hline Tamarack & 17,067 & 17,772 & 18,679 & 6,886 & 29,083 & 18,204 & 506 \\
\hline Oak-hickory & 138,917 & 92,210 & 78,731 & 82,756 & 45,926 & 5,267 & - \\
\hline Elm-ash-cottonwood & 92,916 & 65,691 & 81,035 & 63,515 & 30,253 & 24,505 & -- \\
\hline Maple-basswood & 206,122 & 181,780 & 93,862 & 101,704 & 64,500 & 38,253 & -- \\
\hline Aspen & 472,625 & 144,095 & 68,134 & 20,870 & 3,743 & 2,489 & - \\
\hline Paper birch & 118,869 & 84,430 & 51,101 & 14,231 & 7,219 & -- & -- \\
\hline Balsam poplar & 46,627 & 27,224 & 10,434 & 1,488 & -- & -- & -- \\
\hline Nonstocked & -- & 99 & -- & -- & -- & -- & -- \\
\hline All types & $1,415,154$ & 921,481 & 568,699 & 501,569 & 259,189 & 185,475 & 3,113 \\
\hline
\end{tabular}


Table 40.--Net volume of sawtimber on commercial forest land by forest type and stand-age class, Minnesota, 1977

(In thousand board feet) 1 /

\begin{tabular}{|c|c|c|c|c|c|c|c|}
\hline Forest & & & & Stand-age & class (year & & \\
\hline type & All ages & $0-10$ & $11-20$ & $21-30$ & $31-40$ & $41-50$ & $51-60$ \\
\hline Jack pine & 1,288, & 9,343 & 6,218 & 22,324 & 199,816 & 312,645 & 416,610 \\
\hline Red & & 11,046 & 11,6 & 6,336 & 75,180 & 98,259 & 112,932 \\
\hline White pine & 491,854 & & 3,736 & & 3,307 & 40,202 & 32,307 \\
\hline Balsam fir & $1,250,440$ & 31,237 & 39,819 & 55,218 & 84,935 & 300,842 & 368,555 \\
\hline White spruce & 133,068 & - & & 4,544 & 6,443 & 18,527 & 29,463 \\
\hline Black spruce & 404,865 & 3,928 & 9,103 & 13,554 & 22,714 & 49,318 & 86,363 \\
\hline Northern white-cedar & 872,938 & 1,441 & 2,078 & 5,006 & 12,418 & 21,666 & 39,144 \\
\hline Tamarack & 233,050 & 2,664 & 15,628 & 6,290 & 4,625 & 16,338 & 49,937 \\
\hline oak-hickory & $2,337,948$ & 12,967 & 28,655 & 4,203 & 95,337 & 221,443 & 330,599 \\
\hline Elm-ash-cottonwood & $1,526,492$ & 13,316 & 27,471 & 24,880 & 38,480 & $\begin{array}{l}150,895 \\
\text { las }\end{array}$ & 253,577 \\
\hline Maple-basswood & $3,896,085$ & 26,002 & 29,931 & 24,429 & 155,330 & 348,427 & 655,637 \\
\hline Aspen & $8,336,377$ & 279,718 & 193,790 & 285,176 & $1,006,672$ & $2,267,161$ & $2,393,445$ \\
\hline Paper birch & $1,473,241$ & 4,202 & 18,308 & 25,903 & 121,683 & 308,558 & 337,528 \\
\hline Bals & 829,1 & 18,472 & 22,751 & 11,642 & 84,769 & 232,380 & 183,650 \\
\hline Nonstocked & & & 417 & 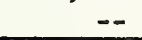 & -- & 895 & - \\
\hline All types & $24,607,197$ & 446,931 & 409,567 & 489,505 & $1,911,709$ & $4,387,556$ & $5,289,747$ \\
\hline
\end{tabular}

1/ International $1 / 4$-inch rule.

(Table 40 continued)

\begin{tabular}{|c|c|c|c|c|c|c|c|}
\hline \multirow{2}{*}{$\begin{array}{l}\text { Forest } \\
\text { type }\end{array}$} & \multicolumn{7}{|c|}{ Stand-age class (years) } \\
\hline & $61-70$ & $71-80$ & $81-90$ & $91-100$ & $101-120$ & $121-140$ & $141+$ \\
\hline Jack pine & 219,381 & 37,222 & 32,780 & 25,228 & 7,302 & -- & -- \\
\hline Red pine & 81,587 & 628,588 & 134,002 & 325,654 & 12,882 & - & -- \\
\hline White pine & 66,361 & 116,519 & 16,464 & 136,463 & 76,495 & -- & -- \\
\hline Balsam fir & 209,129 & 68,266 & 30,755 & 52,692 & 8,992 & -- & -- \\
\hline White spruce & 18,619 & 5,938 & 16,568 & 28,260 & 4,706 & -- & -- \\
\hline Black spruce & 40,053 & 97,774 & 40,778 & 23,422 & 8,775 & 9,083 & -- \\
\hline Northern white-ceda & 78,320 & 69,199 & 127,068 & 102,695 & 121,903 & 282,482 & 9,518 \\
\hline Tamarack & 20,194 & 30,466 & 24,210 & 9,891 & 37,176 & 15,631 & -- \\
\hline 0ak-hickory & 429,357 & 317,402 & 318,135 & 356,212 & 200,068 & 23,570 & -- \\
\hline Elm-ash-cottonwood & 240,906 & 195,014 & 208,672 & 200,558 & 79,853 & 92,870 & -- \\
\hline Maple-basswood & 732,621 & 738,672 & 390,415 & 353,538 & 291,066 & 150,017 & -- \\
\hline Aspen & $1,175,188$ & 414,623 & 238,771 & 64,547 & 9,726 & 7,560 & -- \\
\hline Paper birch & 213,109 & 207,572 & 169,931 & 45,217 & 21,230 & -- & $-\infty$ \\
\hline Balsam poplar & 144 & 88,977 & 36,899 & 4,883 & -- & -- & -- \\
\hline Nonstocked & -- & 736 & -- & -- & -- & -- & -- \\
\hline All types & $3,669,601$ & $3,016,968$ & $1,785,448$ & $1,729,260$ & 880,174 & 581,213 & 9,518 \\
\hline
\end{tabular}


Table 41.--Net volume of sawtimber on commercial forest land by species group and butt log-grade class, Minnesota, 1977

(In thousand board feet) 1 /

\begin{tabular}{|c|c|c|c|c|c|}
\hline Species & $\begin{array}{l}\text { AlT } \\
\text { grades }\end{array}$ & 1 & $\frac{\log 9}{2}$ & 3 & $\begin{array}{l}\text { Tie and } \\
\text { timber }\end{array}$ \\
\hline \multicolumn{6}{|l|}{ SOFTWOODS: } \\
\hline White pine & $1,068,372$ & 211,735 & 181,862 & 647,933 & 26,842 \\
\hline Red pine & $1,952,896$ & 294,630 & 386,324 & $1,271,684$ & 258 \\
\hline Jack pine & $1,566,444$ & 3,234 & 84,359 & $1,478,761$ & 90 \\
\hline White spruce & 648,730 & 3,319 & 26,715 & 618,550 & 146 \\
\hline Black spruce & 460,401 & 4,248 & 33,422 & 422,653 & 78 \\
\hline Balsam fir & $1,338,249$ & & 28,214 & $1,310,035$ & -- \\
\hline Tamarack & 346,508 & 1,550 & 26,669 & 318,253 & 36 \\
\hline Northern whi te-cedar & $1,142,761$ & 14,633 & 74,190 & $1,053,938$ & -- \\
\hline Other so ftwoods & 6,228 & 29 & 46 & 6,153 & -- \\
\hline Total softwoods & $8,530,589$ & 533,378 & 841,801 & $7,127,960$ & 27,450 \\
\hline \multicolumn{6}{|l|}{ HARDWOODS: } \\
\hline Select white oak & $1,076,044$ & 75,495 & 229,741 & 660,706 & 110,102 \\
\hline Select red oak & $1,763,622$ & 149,129 & 443,202 & $1,074,625$ & 96,666 \\
\hline other red oak & 56,414 & 3,025 & 13,976 & 30,339 & 9,074 \\
\hline Hickory & 32,383 & 180 & 9,797 & 21,174 & 1,232 \\
\hline Yellow birch & 39,926 & 6,015 & 11,149 & 22,709 & 53 \\
\hline Hard maple & 489,619 & 35,997 & 93,628 & 337,986 & 22,008 \\
\hline Soft maple & 281,998 & 24,428 & 63,439 & 190,360 & 3,771 \\
\hline Ash & 993,952 & 93,017 & 409,394 & 464,268 & 27,273 \\
\hline Bal sam poplar & $1,101,406$ & 53,550 & 279,463 & 694,769 & 73,624 \\
\hline Paper birch & $1,234,388$ & 75,719 & 365,594 & 739,461 & 53,614 \\
\hline Bigtooth aspen & 303,391 & 17,251 & 62,675 & 206,132 & 17,333 \\
\hline Quaking aspen & $5,671,280$ & 159,061 & $1,121,044$ & $3,992,697$ & 398,478 \\
\hline Basswood & $1,116,835$ & 207,585 & 480,260 & 417,441 & 11,549 \\
\hline Elm & $1,607,307$ & 251,297 & 554,755 & 761,341 & 39,914 \\
\hline Select hardwoods & 40,192 & 1,912 & 7,413 & 30,867 & -- \\
\hline other hardwoods & 267,851 & 69,418 & 51,713 & 146,469 & 251 \\
\hline Noncommercial species & & & & - & -- \\
\hline Total & $16,076,608$ & $1,223,079$ & $4,197,243$ & $9,791,344$ & 864,942 \\
\hline All species & $24,607,197$ & $1,756,457$ & $5,039,044$ & $16,919,304$ & 892,392 \\
\hline
\end{tabular}

1/International $1 / 4$-inch rule. 


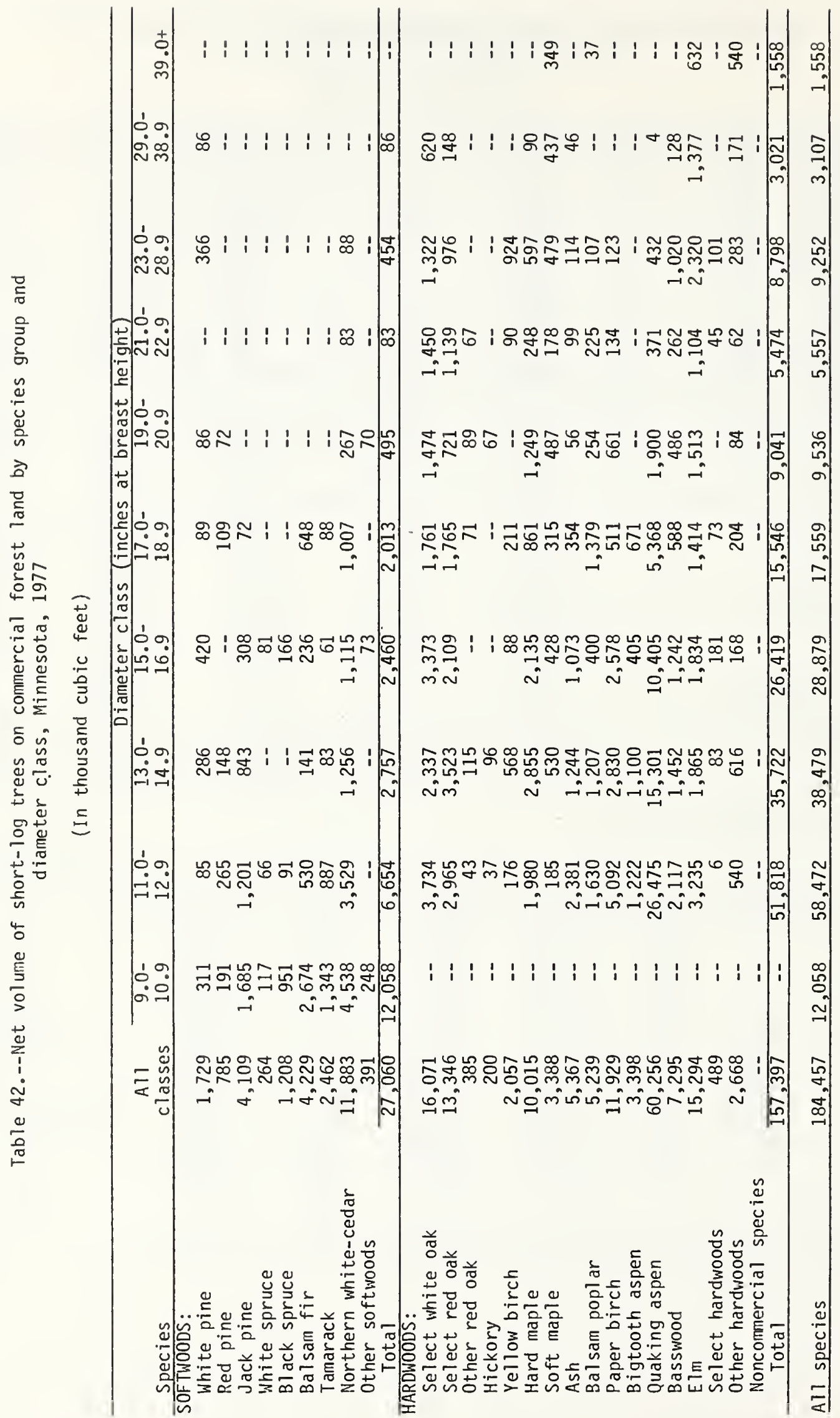




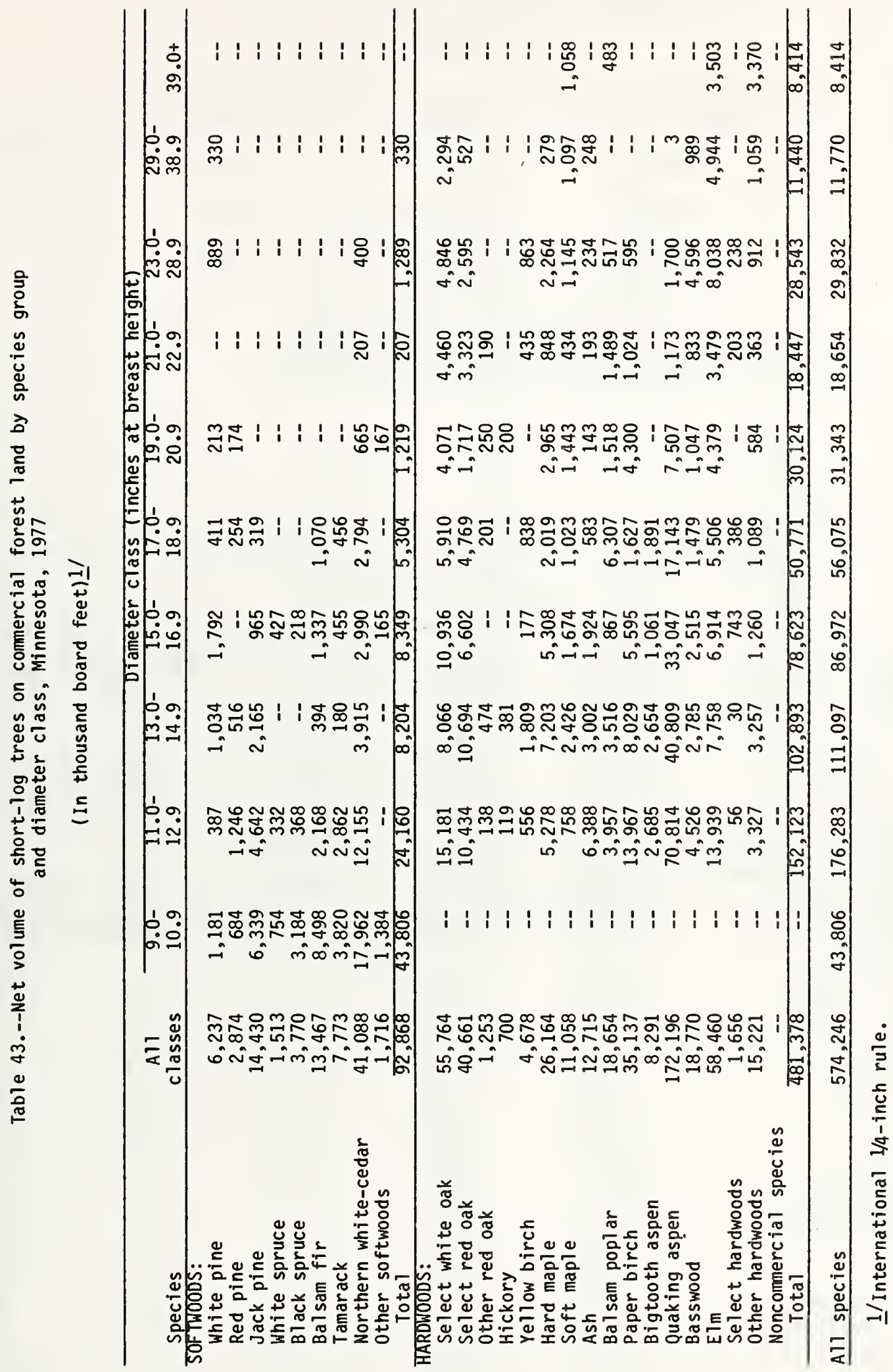


Table 44.--Net annual growth of growing stock on commercial forest 1 and by softwoods and hardwoods, Minnesota, 19621/ and 1976

(In thousand cubic feet)

\begin{tabular}{lcc}
\hline Species & 1962 & 1976 \\
\hline Softwoods: & 106,789 & 119,781 \\
Hardwoods: & 257,420 & 229,139 \\
\cline { 2 - 3 } A11 species & 364,209 & 348,920 \\
\hline
\end{tabular}

1 /Figures have been adjusted from

those publ ished after the 1962 survey to

conform to 1977 vol umes because of changes

in survey definitions and procedures.

Table 45.--Net annual growth of growing stock on commercial forest land by species group and Forest Survey Unit, Minnesota, 1976

(In thousand cubic feet)

\begin{tabular}{|c|c|c|c|c|c|}
\hline Species & $\begin{array}{c}\text { AlT } \\
\text { units }\end{array}$ & $\begin{array}{l}\text { Aspen- } \\
\text { Birch } \\
\end{array}$ & $\begin{array}{c}\text { Northern } \\
\text { Pine }\end{array}$ & $\begin{array}{l}\text { Central } \\
\text { Hardwood }\end{array}$ & Prairie \\
\hline \multicolumn{6}{|l|}{ SOFTWOODS: } \\
\hline White pine & 5,599 & 2,367 & 2,779 & 453 & -- \\
\hline Red pine & 15,684 & 5,811 & 8,742 & 1,113 & 18 \\
\hline Jack pine & 25,677 & 5,799 & 19,216 & 649 & 13 \\
\hline White spruce & 10,701 & 7,328 & 3,228 & 126 & 19 \\
\hline Bl ack spruce & 16,520 & 11,358 & 4,947 & 211 & 4 \\
\hline Bal sam fir & 31,902 & 17,912 & 13,570 & 402 & 18 \\
\hline Tamarack & 2,269 & $(-) 343$ & 2,092 & 508 & 12 \\
\hline Northern white-cedar & 11,274 & 6,747 & 4,527 & -- & -- \\
\hline Other softwoods & 155 & 3 & 22 & 100 & 30 \\
\hline Total & $\overline{119,781}$ & 56,982 & 59,123 & 3,562 & 114 \\
\hline \multicolumn{6}{|l|}{ HARDW00DS: } \\
\hline Select white oak & 8,733 & 140 & 3,213 & 4,294 & 1,086 \\
\hline Select red oak & 15,691 & 191 & 6,034 & 9,025 & 441 \\
\hline Other red oak & 256 & -- & 29 & 214 & 13 \\
\hline Hickory & 455 & -- & 12 & 429 & 14 \\
\hline Yellow birch & 118 & 30 & 85 & 3 & -- \\
\hline Hard maple & 8,660 & 1,298 & 2,535 & 4,335 & 492 \\
\hline Soft maple & 6,454 & 1,574 & 1,813 & 2,940 & 127 \\
\hline Ash & 9,468 & 3,996 & 3,621 & 1,538 & 313 \\
\hline Balsam poplar & 17,757 & 6,229 & 9,429 & 833 & 1,266 \\
\hline Paper birch & 30,290 & 10,619 & 15,829 & 3,761 & 81 \\
\hline Bigtooth aspen & 1,086 & 409 & 341 & 332 & 4 \\
\hline Quaking aspen & 93,083 & 31,322 & 45,739 & 11,430 & 4,592 \\
\hline Basswood & 17,252 & 891 & 6,228 & 8,516 & 1,617 \\
\hline Elm & 18,022 & 1,248 & 5,617 & 10,307 & 850 \\
\hline Select hardwoods & 440 & 4 & 73 & 306 & 57 \\
\hline Other hardwoods & 1,374 & $(-) 34$ & 71 & 470 & 867 \\
\hline Noncommercial species & -- & -- & -- & -- & -- \\
\hline Total & 229,139 & 57,917 & 100,669 & 58,733 & 11,820 \\
\hline All species & 348,920 & 114,899 & 159,792 & 62,295 & 11,934 \\
\hline
\end{tabular}


Table 46.--Net annual growth of sawtimber on commercial forest land by species group and Forest Survey Unit, Minnesota, 1976

(In thousand board feet) $1 /$

\begin{tabular}{|c|c|c|c|c|c|}
\hline Species & $\begin{array}{l}\text { AाT } \\
\text { units }\end{array}$ & $\begin{array}{l}\text { Aspen- } \\
\text { Birch }\end{array}$ & $\begin{array}{c}\text { Northern } \\
\text { Pine }\end{array}$ & $\begin{array}{l}\text { Central } \\
\text { Hardwood }\end{array}$ & Prairie \\
\hline \multicolumn{6}{|l|}{ SOFTWOODS: } \\
\hline White pine & 33,304 & 15,969 & 14,775 & 2,560 & -- \\
\hline Red pine & 74,768 & 25,414 & 48,197 & 1,064 & 93 \\
\hline Jack pine & 88,650 & 30,665 & 56,971 & 981 & 33 \\
\hline White spruce & 36,750 & 23,581 & 12,764 & .347 & 58 \\
\hline Black spruce & 18,568 & 14,758 & 3,800 & 10 & -- \\
\hline Balsam fir & 86,053 & 48,647 & 36,542 & 864 & -- \\
\hline Tamarack & 8,460 & & 5,698 & 2,743 & 13 \\
\hline Northern white-cedar & 33,003 & 19,486 & 13,517 & $=-$ & $=-$ \\
\hline Other softwoods & 763 & - & 664 & 71 & 28 \\
\hline Total & 380,319 & 178,526 & 192,928 & 8,640 & 225 \\
\hline \multicolumn{6}{|l|}{ HARDWOODS: } \\
\hline Select white oak & 30,845 & 199 & 9,585 & 16,546 & 4,515 \\
\hline Select red oak & 61,191 & 658 & 19,759 & 38,201 & 2,573 \\
\hline other red oak & 960 & -- & 3 & 909 & 48 \\
\hline Hickory & 1,484 & -- & 6 & 1,456 & 22 \\
\hline Yellow birch & 612 & 428 & 166 & 18 & -- \\
\hline Hard maple & 30,649 & 2,639 & 6,239 & 19,508 & 2,263 \\
\hline Soft maple & 18,380 & 1,574 & 3,278 & 10,481 & 3,047 \\
\hline Ash & 18,003 & 6,377 & 5,340 & 5,078 & 1,208 \\
\hline Balsam poplar & 51,922 & 22,274 & 25,742 & 812 & 3,094 \\
\hline Paper birch & 53,434 & 23,611 & 26,074 & 3,715 & 34 \\
\hline Bigtooth aspen & 11,116 & 1,130 & 10,651 & $1-\hat{665}$ & $=-$ \\
\hline Quaking aspen & 330,071 & 132,104 & 157,084 & 34,262 & 6,621 \\
\hline Basswood & 48,788 & 2,452 & 12,042 & 27,208 & 7,086 \\
\hline Elm & 61,699 & 6,113 & 18,524 & 34,852 & 2,210 \\
\hline Select hardwoods & 2,261 & -- & 444 & 1,564 & 253 \\
\hline other hardwoods & 9,730 & $(-) 19$ & 287 & 3.358 & 6,104 \\
\hline Noncommercial species & & -- & -- & -- & - \\
\hline Total & 731,145 & 199,540 & 295,224 & 197,303 & 39,078 \\
\hline All species & $1,111,464$ & 378,066 & 488,152 & 205,943 & 39,303 \\
\hline
\end{tabular}

1/International $1 / 4$-inch rule. 


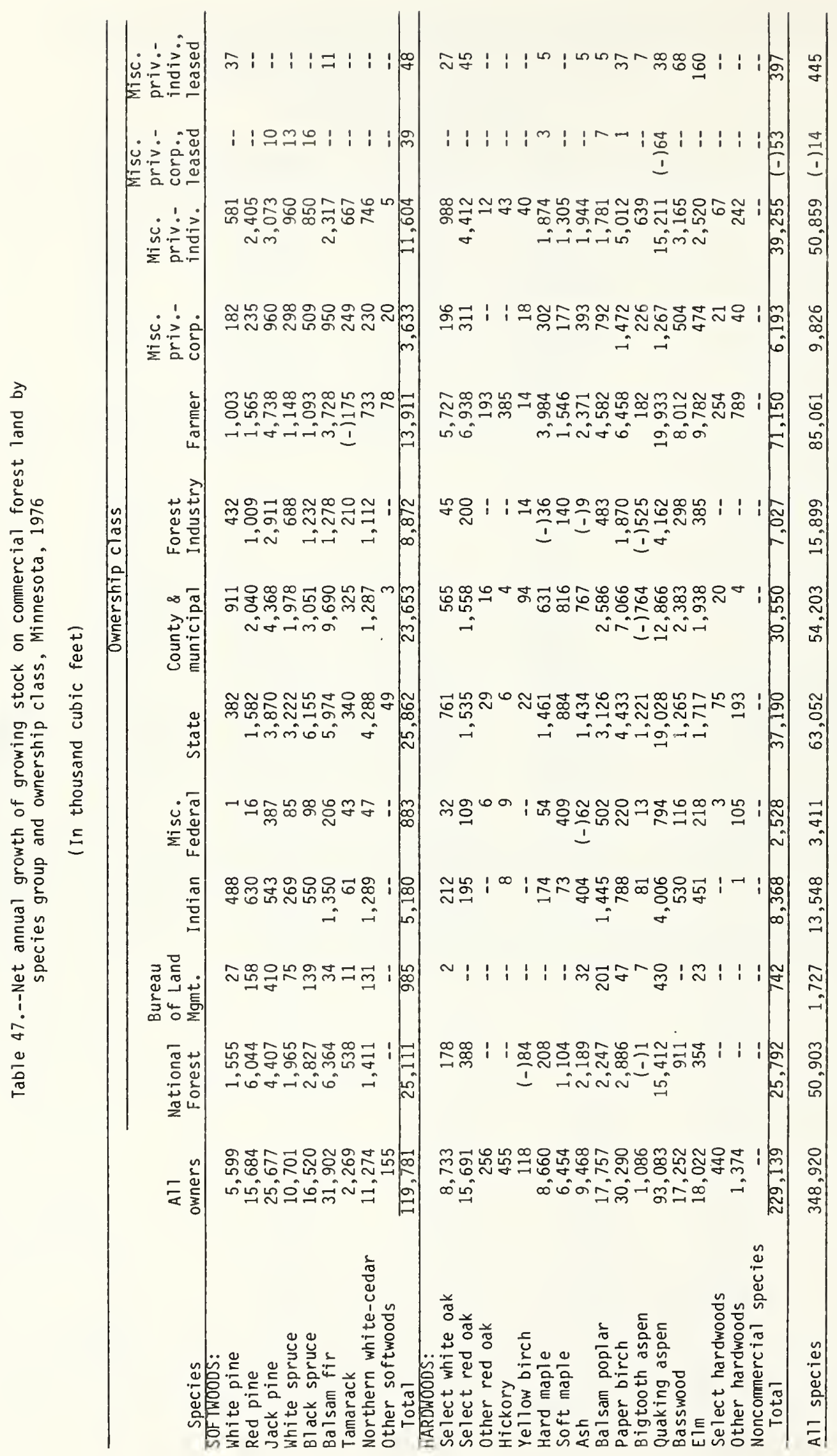


Table 49.--Net annual growth of growing stock on commercial forest land by species group and forest type, Minnesota, 1976

(In thousand cubic feet)

\begin{tabular}{|c|c|c|c|c|c|c|c|c|}
\hline \multirow[b]{2}{*}{ Species } & \multirow[b]{2}{*}{$\begin{array}{c}\text { All } \\
\text { types }\end{array}$} & \multicolumn{7}{|c|}{ Forest type } \\
\hline & & $\begin{array}{l}\text { Jack } \\
\text { pine }\end{array}$ & $\begin{array}{l}\text { Red } \\
\text { pine }\end{array}$ & $\begin{array}{l}\text { White } \\
\text { pine }\end{array}$ & $\begin{array}{c}\text { Balsam } \\
\text { fir }\end{array}$ & $\begin{array}{l}\text { White } \\
\text { spruce }\end{array}$ & $\begin{array}{l}\text { Black } \\
\text { spruce }\end{array}$ & $\begin{array}{c}\text { Northern } \\
\text { white-cedar }\end{array}$ \\
\hline SOF TWOODS: & & & & & & & & \\
\hline White pine & 5,599 & 119 & 1,168 & 1,247 & 320 & 35 & 89 & 37 \\
\hline Red pine & 15,684 & 1,780 & 8,797 & 387 & 346 & 42 & 48 & 59 \\
\hline Jack pine & 25,677 & 20,768 & 667 & 111 & 172 & 78 & 405 & 87 \\
\hline White spruce & 10,701 & 226 & 266 & 267 & 1,517 & 1,190 & 464 & 34 \\
\hline Black spruce & 16,520 & 438 & 54 & $(-) 67$ & 2,060 & & 11,517 & 168 \\
\hline Balsam fir & 31,902 & 375 & 564 & $(-) 532$ & 8,260 & 188 & 1,032 & 169 \\
\hline Tamarack & 2,269 & 5 & 14 & -- & 129 & $(-) 819$ & 732 & 84 \\
\hline Northern white-cedar & 11,274 & -- & 11 & 8 & 1,334 & 43 & 376 & 6,959 \\
\hline other softwoods & 155 & -- & 25 & -- & - & -- & 10 & -- \\
\hline Total & 119,781 & 23,711 & 11,566 & 1,421 & 14,138 & 804 & 14,673 & 7,597 \\
\hline HARDWOOOS: & & & & & & & & \\
\hline Select white oak & 8,733 & 31 & 8 & 14 & 18 & 1 & - & -- \\
\hline Select red oak & 15,691 & 94 & 33 & 15 & 4 & - & 6 & -- \\
\hline Other red oak & 256 & -- & -- & -- & -- & -- & -- & -- \\
\hline Hickory & 455 & -- & -- & -- & -- & -- & -- & -- \\
\hline Yellow birch & 118 & -- & -- & -- & 7 & -- & -- & 15 \\
\hline Hard maple & 8,660 & -- & -- & 3 & 19 & - & - & - \\
\hline Soft maple & 6,454 & 146 & 3 & 41 & 44 & -- & 6 & 1 \\
\hline Ash & 9,468 & 2 & 6 & 13 & 261 & $(-) 310$ & 50 & $(-) 24$ \\
\hline Balsam poplar & 17,757 & 33 & 36 & 4 & $(-) 819$ & 34 & 40 & 198 \\
\hline Paper birch & 30,290 & 495 & 411 & $(-) 24$ & 2,344 & 296 & 239 & 150 \\
\hline Bigtooth aspen & 1,086 & $(-) 576$ & $(-) 118$ & 1 & 14 & - & 88 & 10 \\
\hline Quaking aspen & 93,083 & 1,655 & $(-) 30$ & 196 & 2,261 & 480 & 1,143 & 117 \\
\hline Basswood & 17,252 & 5 & -- & 11 & 36 & -- & -- & -- \\
\hline $\mathrm{E} 1 \mathrm{~m}$ & 18,022 & 8 & 3 & -- & 69 & $(-) 129$ & 6 & 5 \\
\hline Select hardwoods & 440 & -- & -- & -- & -- & -- & - & -- \\
\hline Other hardwoods & 1,374 & 12 & 4 & -- & -- & -- & -- & -- \\
\hline Noncommercial species & - & -- & -- & -- & -- & - & -- & -- \\
\hline Total & 229,139 & 1,905 & 356 & 274 & 4,258 & 372 & 1,578 & 472 \\
\hline All species & 348,920 & 25,616 & 11,922 & 1,695 & 18,396 & 1,176 & 16,251 & 8,069 \\
\hline
\end{tabular}

(Table 49 continued)

\begin{tabular}{|c|c|c|c|c|c|c|c|c|}
\hline \multirow{3}{*}{ Species } & \multicolumn{8}{|c|}{ Forest type } \\
\hline & Tamarack & $\begin{array}{c}\text { 0ak- } \\
\text { hickory }\end{array}$ & $\begin{array}{l}\text { Elm-ash- } \\
\text { cottonwood }\end{array}$ & $\begin{array}{l}\text { Maple- } \\
\text { basswood }\end{array}$ & Aspen & $\begin{array}{l}\text { Paper } \\
\text { birch }\end{array}$ & $\begin{array}{l}\text { Balsam } \\
\text { poplar }\end{array}$ & $\begin{array}{l}\text { Non- } \\
\text { stocked }\end{array}$ \\
\hline & & & & & & & & \\
\hline White pine & 54 & 87 & 53 & 332 & 1,558 & 491 & 9 & -- \\
\hline Red pine & 13 & 245 & 14 & 20 & 3,172 & 745 & 16 & -- \\
\hline Jack pine & 32 & 252 & 21 & 1 & 2,687 & 292 & 22 & 82 \\
\hline White spruce & 52 & $(-) 49$ & 149 & 318 & 5,143 & 816 & 308 & -- \\
\hline Bl ack spruce & 840 & -- & 54 & $(-) 17$ & 1,338 & $(-14$ & 78 & 14 \\
\hline Balsam fir & 133 & 30 & 584 & 554 & 14,662 & 4,339 & 1,335 & 209 \\
\hline Tamarack & 2,690 & 4 & $(-) 402$ & 109 & $(-) 240$ & $(-) 104$ & 37 & 30 \\
\hline Northern white-cedar & 192 & -- & 882 & 130 & 1,107 & 2 & 219 & 11 \\
\hline Other softwoods & -- & 71 & 4 & 28 & - & 9 & 8 & -- \\
\hline Total & 4,006 & 640 & 1,359 & 1,475 & 29,427 & 6,586 & 2,032 & 346 \\
\hline HAROWOOOS: & & & & & & & & \\
\hline Select white oak & -- & 3,983 & 174 & 1,311 & 2,895 & 245 & 48 & 5 \\
\hline Select red oak & 7 & 8,207 & 348 & 1,759 & 4,293 & 906 & 19 & -- \\
\hline Other red oak & - & 172 & 4 & 38 & 38 & 1 & 3 & - \\
\hline Hickory & -- & 363 & -- & 75 & -- & 17 & -. & -- \\
\hline Yellow birch & -- & -. & 36 & 20 & 7 & 31 & 2 & -- \\
\hline Hard maple & -. & 390 & 249 & 6,464 & 1,212 & 269 & 52 & 2 \\
\hline Soft maple & 14 & 275 & 882 & 1,197 & 2,914 & 916 & 15 & -- \\
\hline Ash & 16 & 240 & 4,202 & 1,317 & 2,895 & 399 & 385 & 16 \\
\hline Balsam poplar & 121 & 95 & 556 & $(-) 293$ & 7,395 & 616 & 9,819 & $(-) 78$ \\
\hline Paper birch & 80 & 1,486 & 370 & 1,669 & 10,854 & 11,349 & 528 & 43 \\
\hline Bigtooth aspen & -- & 351 & 32 & $(-) 88$ & 1,977 & $(-), 618$ & 13 & $\ldots$ \\
\hline Quaking aspen & $(-) 47$ & 1,033 & 236 & 1,609 & 81,400 & 921 & 2,033 & 76 \\
\hline Basswood & - & 1,772 & 442 & 11,089 & 3,315 & 566 & 16 & -- \\
\hline Elm & 86 & 1,632 & 2,267 & 8,762 & 3,871 & 762 & 646 & 34 \\
\hline Select hardwoods & -- & 132 & 26 & 162 & 98 & 16 & 3 & 3 \\
\hline other hardwoods & - & 139 & 933 & 187 & 80 & -- & 13 & 6 \\
\hline Noncommercial species & -- & - & - & - & -- & -- & - & -- \\
\hline Total & 277 & 20,270 & 10,751 & 35,278 & 123,244 & 16,396 & 13,595 & 107 \\
\hline All species & 4,283 & 20,910 & 12,116 & 36,753 & 152,671 & 22,982 & 15,627 & 453 \\
\hline
\end{tabular}


Table 50.--Net annual growth of sawtimber on commercial forest land by species group and forest type, Minnesota, 1976

(In thousand board feet) $\underline{1}$ /

\begin{tabular}{|c|c|c|c|c|c|c|c|c|}
\hline & & & & & Forest & type & & \\
\hline Species & $\begin{array}{c}\text { All } \\
\text { types }\end{array}$ & $\begin{array}{l}\text { Jack } \\
\text { pine }\end{array}$ & $\begin{array}{l}\text { Red } \\
\text { pine }\end{array}$ & $\begin{array}{l}\text { White } \\
\text { pine }\end{array}$ & $\begin{array}{l}\text { Balsam } \\
\text { fir }\end{array}$ & $\begin{array}{l}\text { White } \\
\text { spruce }\end{array}$ & $\begin{array}{l}\text { Black } \\
\text { spruce }\end{array}$ & $\begin{array}{l}\text { Northern } \\
\text { white-cedar }\end{array}$ \\
\hline SOF TWOODS: & & & & & & & & \\
\hline White pine & 33,304 & 356 & 8,612 & 9,399 & 1,653 & 215 & 497 & 255 \\
\hline Red pine & 74,768 & 6,511 & 40,989 & 2,353 & 1,539 & 104 & 204 & 636 \\
\hline Jack pine & 88,650 & 62,359 & 1,673 & 215 & 544 & 193 & 3,219 & 375 \\
\hline White spruce & 36,750 & 365 & 1,008 & 2,110 & 5,639 & 3,452 & 897 & $(-) 24$ \\
\hline Black spruce & 18,568 & 670 & 16 & 30 & 4,272 & 23 & 4,961 & $(-) 188$ \\
\hline Balsam fir & 86,053 & 166 & 2,183 & 210 & 19,181 & 482 & 2,032 & 483 \\
\hline Tamarack & 8,460 & 24 & 10 & - & 1,201 & $(-) 2,795$ & 2,718 & 240 \\
\hline Northern white-cedar & 33,003 & -- & 36 & 8 & 4,679 & 170 & 1,674 & 11,945 \\
\hline 0ther sof twoods & 763 & -- & -- & -- & - & -- & -- & -- \\
\hline Total & 380,319 & 70,451 & 54,527 & 14,325 & 38,708 & 1,844 & 16,202 & 13,722 \\
\hline HARDWOODS: & & & & & & & & \\
\hline Select white oak & 30,845 & 7 & -- & 13 & 36 & -- & -- & -- \\
\hline Select red oak & 61,191 & 37 & 43 & 62 & 5 & -- & -- & -- \\
\hline other red oak & 960 & - & - & - & -- & -- & -- & -- \\
\hline Hickory & 1,484 & -- & -- & -- & -- & -- & -- & -- \\
\hline Yellow birch & 612 & -- & -- & -- & 6 & -- & -- & 24 \\
\hline Hard maple & 30,649 & -- & -- & 17 & -- & -- & -- & -- \\
\hline Soft maple & 18,380 & -- & -- & 6 & 770 & -- & 9 & -- \\
\hline Ash & 18,003 & -- & -- & 9 & 303 & $(-) 1,516$ & 114 & 130 \\
\hline Balsam poplar & 51,922 & 42 & -- & -- & 1,140 & 14 & 22 & 971 \\
\hline Paper birch & 53,434 & 1,062 & 1,607 & 42 & 3,917 & 400 & 33 & 115 \\
\hline Bigtooth aspen & 11,116 & 26 & 5 & -- & 24 & -- & 7 & 10 \\
\hline Quaking aspen & 330,071 & 3,498 & 1,341 & $(-) 355$ & 6,006 & 346 & 2,877 & 266 \\
\hline Basswood & 48,788 & - & -- & 25 & 88 & -- & - & - \\
\hline Elm & 61,699 & 13 & -- & -- & 133 & $(-) 520$ & -- & 21 \\
\hline Select hardwoods & 2,261 & -- & -- & -- & -- & -- & -- & -- \\
\hline other hardwoods & 9,730 & -- & -- & -- & -. & -- & -- & -- \\
\hline Noncommercial species & - & -- & -- & -- & -- & -- & -- & -- \\
\hline Total & 731,145 & 4,685 & 2,996 & $T-1181$ & 12,429 & $T-11,276$ & 3,061 & 1,537 \\
\hline All species & $1,111,464$ & 75,136 & 57,523 & 14,144 & 51,136 & 568 & 19,264 & 15,259 \\
\hline
\end{tabular}

1/International $1 / 4-$ inch rule. 


\begin{tabular}{|c|c|c|c|c|c|c|c|c|}
\hline \multirow[b]{2}{*}{ Species } & \multicolumn{8}{|c|}{ Forest type } \\
\hline & Tamarack & $\begin{array}{c}\text { Oak- } \\
\text { hickory }\end{array}$ & $\begin{array}{l}\text { Elm-ash- } \\
\text { cottonwood }\end{array}$ & $\begin{array}{l}\text { Maple- } \\
\text { basswood }\end{array}$ & Aspen & $\begin{array}{l}\text { Paper } \\
\text { birch }\end{array}$ & $\begin{array}{l}\text { Batsam } \\
\text { poplar }\end{array}$ & $\begin{array}{c}\text { Non- } \\
\text { stocked }\end{array}$ \\
\hline \multicolumn{9}{|l|}{ SOFTWOODS: } \\
\hline White pine & 259 & 445 & 277 & 1,667 & 7,179 & 2,435 & 55 & -- \\
\hline Red pine & 61 & 765 & 24 & 113 & 17,685 & 3,705 & 79 & -- \\
\hline Jack pine & 50 & 325 & & $(-) 18$ & 18,930 & 629 & $(-) 158$ & 276 \\
\hline White spruce & 85 & $(-) 365$ & 883 & 1,330 & 15,688 & 3,970 & 1,712 & -- \\
\hline Bl ack spruce & 348 & -- & & 715 & 7,318 & 212 & 129 & 24 \\
\hline Balsam fir & 173 & 14 & $(-) 342$ & 6,472 & 39,017 & 9,676 & 5,446 & 860 \\
\hline Tamarack & 7,270 & 26 & $(-) 632$ & 192 & $(-) 972$ & 650 & 494 & 34 \\
\hline Northern white-cedar & 1,333 & -- & 3,734 & 1,111 & 7,752 & $(-) 58$ & 588 & 31 \\
\hline Other softwoods & -- & 69 & 15 & 30 & -- & -- & 649 & -- \\
\hline \multirow{2}{*}{\multicolumn{9}{|c|}{ HARDWOODS: }} \\
\hline & & & & & & & & \\
\hline Select white oak & -- & 13,612 & 559 & 7,536 & 8,107 & 882 & 81 & 12 \\
\hline Select red oak & 39 & 34,649 & 20 & 6,967 & 15,184 & 4,120 & 65 & -- \\
\hline Other red oak & -- & 702 & 23 & 187 & 31 & - & 17 & -- \\
\hline Hickory & -- & 473 & -- & 949 & -- & 62 & -- & -- \\
\hline Yellow birch & -- & - & 105 & 404 & 7 & 60 & 6 & -- \\
\hline Hard maple & -- & 766 & 215 & 27,199 & 1,900 & 540 & 12 & -- \\
\hline Soft maple & -- & 1,978 & 5,668 & 1,682 & 8,151 & 116 & -- & -- \\
\hline Ash & -- & 279 & 7,714 & 3,970 & 3,567 & 1,486 & 1,915 & 32 \\
\hline Balsam poplar & 141 & 127 & 1,109 & 1,811 & 18,374 & 3,315 & 24,856 & -- \\
\hline Paper birch & 16 & 1,152 & 1,220 & 4,607 & 23,456 & 15,660 & 138 & 9 \\
\hline Bigtooth aspen & -- & 633 & 51 & $(-) 117$ & 11,700 & 11,223 & -- & -- \\
\hline Quaking aspen & 80 & 5,180 & 883 & 5,008 & 286,167 & 8,485 & 10,116 & 173 \\
\hline Basswood & -- & 5,328 & 2,089 & 36,014 & 3,989 & 1,191 & 64 & -- \\
\hline Elm & 29 & 2,575 & 10,634 & 28,735 & 16,165 & 1,828 & 1,990 & 96 \\
\hline Select hardwoods & -- & 1,681 & & 457 & 81 & 33 & -- & -- \\
\hline Other hardwoods & -- & 146 & 5,332 & 4,168 & 66 & -- & -- & 18 \\
\hline Noncormercial species & -- & -- & -- & -- & -- & -- & -- & -- \\
\hline Total & 305 & 69,280 & 35,631 & 129,578 & 396,945 & 36,555 & 39,260 & 340 \\
\hline All species & 9,884 & 70,560 & 39,666 & 141,189 & 509,542 & 57,774 & 48,254 & 1,565 \\
\hline
\end{tabular}


Table 51.--Net annual growth of growing stock on commercial forest 1 and by forest type and stand-age class, Minnesota, 1976

(In thousand cubic feet)

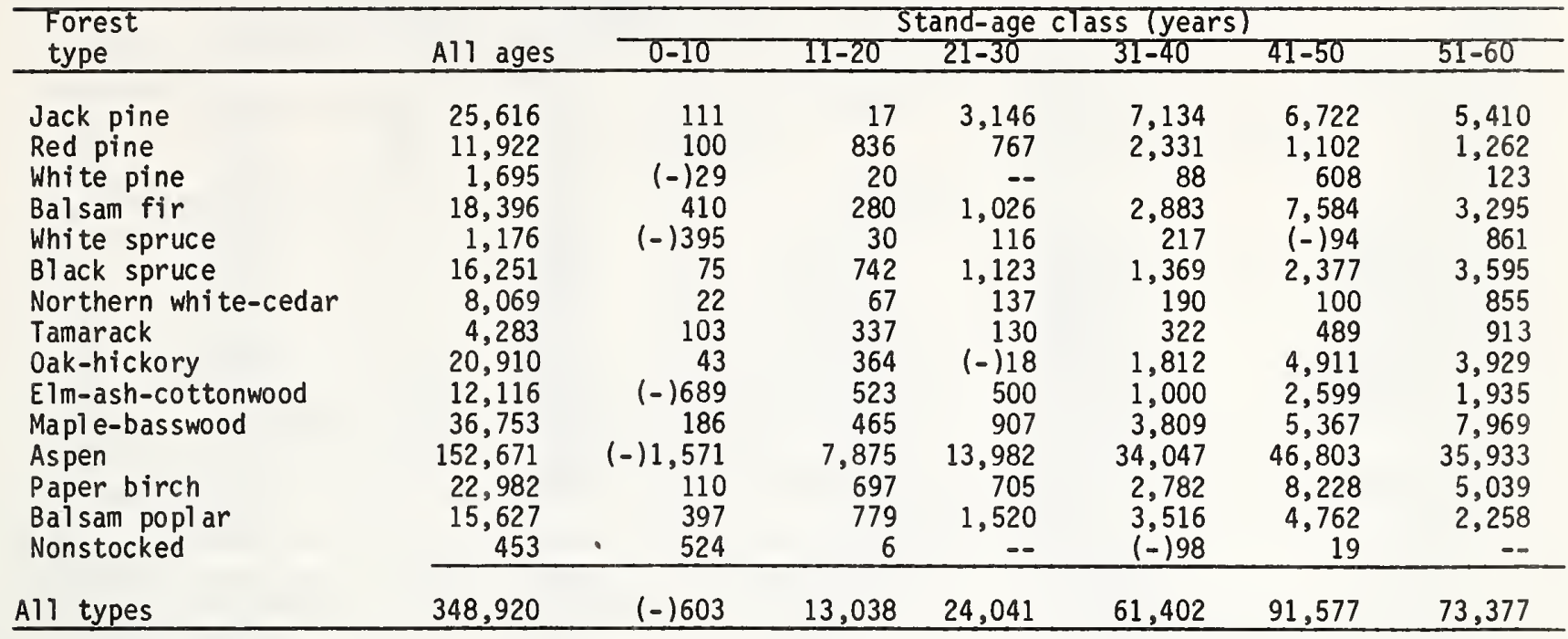

(Table 51 continued)

\begin{tabular}{|c|c|c|c|c|c|c|c|}
\hline Forest & & & & Stand-ag & class (y & rst & \\
\hline Type & $61-70$ & $71-80$ & $81-90$ & $91-100$ & $101-120$ & $121-140$ & $141+$ \\
\hline Jack pine & 2,882 & 284 & $(-) 86$ & $(-) 53$ & 49 & -- & -- \\
\hline Red pine & 649 & 3,084 & 620 & 1,089 & 82 & -- & -- \\
\hline White pine & 479 & 265 & 138 & $(-) 210$ & 213 & -- & -- \\
\hline Bal sam fir & 2,841 & 174 & $(-) 370$ & 173 & 100 & -- & -- \\
\hline White spruce & 191 & 30 & 99 & 101 & 20 & -- & -- \\
\hline Black spruce & 1,930 & 2,233 & 1,313 & 1,019 & 94 & 381 & -- \\
\hline Northen white-cedar & 1,241 & 784 & 1,539 & 942 & 618 & 1,492 & 82 \\
\hline Tamarak & 587 & 306 & $(-) 309$ & 144 & 880 & 369 & 12 \\
\hline Oak-hikory & 3,876 & 2,574 & 557 & 1,809 & 922 & 131 & -- \\
\hline Elm-ash-cottonwood & 1,822 & 588 & 1,760 & 1,131 & 511 & 436 & -- \\
\hline Maple-bas swood & 6,685 & 4,734 & 2,681 & 2,306 & 706 & 938 & -- \\
\hline Aspen & 11,096 & 2,881 & 1,099 & 400 & 65 & 61 & -- \\
\hline Paper birch & 2,662 & 1,797 & 774 & 35 & 153 & -- & -- \\
\hline Balsam poplar & 1,215 & 822 & 318 & 40 & -- & -- & -- \\
\hline Nonstocked & -- & 2 & -- & -- & -- & -- & -- \\
\hline Al1 types & 38,156 & 20,558 & 10,133 & 8,926 & 4,413 & 3,808 & 94 \\
\hline
\end{tabular}


Table 52.--Net annual growth of sawtimber on commercial forest 1 and by forest type and stand-age class, Minnesota, 1976

(In thousand board feet) 1 /

\begin{tabular}{|c|c|c|c|c|c|c|c|}
\hline \multirow{2}{*}{$\begin{array}{l}\text { Forest } \\
\text { type }\end{array}$} & \multirow[b]{2}{*}{ All ages } & \multicolumn{6}{|c|}{ Stand-age class (years) } \\
\hline & & $0-10$ & $11-20$ & $21-30$ & $31-40$ & $41-50$ & $51-60$ \\
\hline $\begin{array}{l}\text { Jack pine } \\
\text { Red pine } \\
\text { White pine } \\
\text { Balsam fir } \\
\text { White spruce } \\
\text { Black spruce } \\
\text { Northern white-cedar } \\
\text { Tamarack } \\
\text { Oak-hickory } \\
\text { Elm-ash-cottonwood } \\
\text { Maple-basswood } \\
\text { Aspen } \\
\text { Paper birch } \\
\text { Balsam poplar } \\
\text { Nonstocked }\end{array}$ & $\begin{array}{r}75,136 \\
57,523 \\
14,144 \\
51,136 \\
568 \\
19,264 \\
15,259 \\
9,884 \\
70,560 \\
39,666 \\
141,189 \\
509,542 \\
57,774 \\
48,254 \\
1,565\end{array}$ & $\begin{array}{r}(-) 567 \\
360 \\
-- \\
1,594 \\
(-) 2,036 \\
94 \\
33 \\
61 \\
(-) 152 \\
(-) 305 \\
2,116 \\
3,970 \\
182 \\
(-) 1,482 \\
1,524\end{array}$ & $\begin{array}{r}719 \\
1,165 \\
131 \\
455 \\
-- \\
777 \\
48 \\
836 \\
754 \\
693 \\
3,654 \\
15,151 \\
435 \\
341 \\
5\end{array}$ & $\begin{array}{r}1,967 \\
372 \\
-- \\
1,331 \\
261 \\
848 \\
103 \\
166 \\
97 \\
1,020 \\
(-) 276 \\
30,531 \\
1,455 \\
375 \\
--\end{array}$ & $\begin{array}{r}15,932 \\
6,891 \\
664 \\
5,471 \\
198 \\
1,528 \\
(-) 1 \\
121 \\
3,270 \\
2,027 \\
12,363 \\
79,956 \\
6,578 \\
7,199 \\
--\end{array}$ & $\begin{array}{r}24,704 \\
4,925 \\
1,310 \\
16,623 \\
(-) 1,866 \\
2,797 \\
(-) 696 \\
1,965 \\
10,004 \\
10,460 \\
13,065 \\
166,023 \\
19,370 \\
18,166 \\
20\end{array}$ & $\begin{array}{r}19,078 \\
5,904 \\
804 \\
16,663 \\
981 \\
6,609 \\
794 \\
3,510 \\
11,277 \\
3,939 \\
29,692 \\
132,793 \\
11,100 \\
9,914 \\
--\end{array}$ \\
\hline All types & $1,111,464$ & 5,392 & 25,164 & 38,250 & 142,197 & 286,870 & 253,058 \\
\hline
\end{tabular}

1/International 1/4-inch rule.

(Table 52 continued)

\begin{tabular}{|c|c|c|c|c|c|c|c|}
\hline Forest & & & & d-age cT & s (years) & & \\
\hline type & $61-70$ & $71-80$ & $81-90$ & $91-100$ & $101-120$ & $121-140$ & $141+$ \\
\hline Jack pine & 9,840 & 1,233 & 986 & 1,121 & 123 & -- & -- \\
\hline Red pie & 2,833 & 20,814 & 4,892 & 8,657 & 710 & -- & -- \\
\hline White pine & 3,019 & 2,586 & 444 & 3,926 & 1,260 & -- & -- \\
\hline Bal sam fir & 5,691 & 3,255 & $(-) 686$ & 509 & 230 & -- & -- \\
\hline White spruce & 1,444 & 117 & 381 & 988 & 100 & -- & -- \\
\hline Black spruce & 1,308 & 2,631 & 774 & 1,603 & 157 & 138 & -- \\
\hline Northen white-cedar & 2,753 & 2,255 & 4,595 & 1,769 & 143 & 3,270 & 193 \\
\hline Tamarak & 494 & $(-) 310$ & 1,208 & 282 & 1,247 & 304 & -- \\
\hline Oak-hikory & 16,819 & 13,071 & 1,890 & 9,428 & 3,551 & 551 & -- \\
\hline Elm-ash-cottonwood & 4,143 & 5,014 & 5,752 & 3,838 & 1,329 & 1,756 & -- \\
\hline Maple-basswood & 21,401 & 26,144 & 13,460 & 9,366 & 6,039 & 4,165 & -- \\
\hline Aspen & 52,045 & 17,277 & 9,437 & 1,778 & 451 & 130 & -- \\
\hline Paper birch & 7,110 & 5,656 & 3,857 & 1,724 & 307 & -- & -- \\
\hline Balsam poplar & 9,142 & 2,709 & 1,361 & 529 & -- & -- & -- \\
\hline Nonstocked & -- & 16 & -- & -- & -- & -- & -- \\
\hline All types & 138,042 & 102,468 & 48,351 & 45,518 & 15,647 & 10,314 & 193 \\
\hline
\end{tabular}


Table 53.--Timber removals $1 /$ from growing stock and sawtimber on commercial forest land by species group, Minnesota, 1962 and 1976

\begin{tabular}{|c|c|c|c|c|}
\hline \multirow[b]{2}{*}{ Species } & \multicolumn{2}{|c|}{ Growing stock } & \multicolumn{2}{|c|}{ Sawtimber } \\
\hline & $\underline{2} / 1962$ & 1976 & $\underline{2 / 1962}$ & 1976 \\
\hline & \multicolumn{2}{|c|}{ Thousand cubic feet } & \multicolumn{2}{|c|}{$3 /$ Thousand board feet } \\
\hline $\begin{array}{l}\text { SOF TWOODS: } \\
\text { Whi te pine } \\
\text { Red pine } \\
\text { Jack pine } \\
\text { Spruce } \\
\text { Balsam fir } \\
\text { Tamarack } \\
\text { Northern white-cedar } \\
\text { Other } \\
\text { Total }\end{array}$ & $\begin{array}{r}3,620 \\
3,416 \\
22,678 \\
20,589 \\
12,601 \\
3,485 \\
2,445 \\
66 \\
68,900\end{array}$ & $\begin{array}{r}2,910 \\
4,177 \\
21,470 \\
17,943 \\
13,893 \\
4,982 \\
3,315 \\
110 \\
68,800\end{array}$ & $\begin{array}{r}20,759 \\
15,976 \\
54,844 \\
45,600 \\
20,860 \\
6,305 \\
4,586 \\
49 \\
168,979\end{array}$ & $\begin{array}{r}13,190 \\
15,214 \\
56,934 \\
38,809 \\
27,000 \\
10,165 \\
8,548 \\
82 \\
169,942\end{array}$ \\
\hline $\begin{array}{l}\text { HARDWOODS: } \\
\text { Whi te oak } \\
\text { Red oak } \\
\text { Yellow birch } \\
\text { Hard maple } \\
\text { Soft maple } \\
\text { Ash } \\
\text { Paper birch } \\
\text { Aspen } \\
\text { Basswood } \\
\text { Elm } \\
\text { Other } \\
\text { Total }\end{array}$ & $\begin{array}{r}4,738 \\
6,743 \\
441 \\
1,036 \\
1,273 \\
2,632 \\
3,408 \\
45,675 \\
2,941 \\
4,180 \\
3,933 \\
7,000\end{array}$ & $\begin{array}{r}5,119 \\
10,102 \\
16 \\
1,388 \\
2,840 \\
5,063 \\
9,113 \\
72,979 \\
3,529 \\
7,813 \\
6,838 \\
124,800\end{array}$ & $\begin{array}{r}12,763 \\
21,149 \\
1,840 \\
2,534 \\
3,737 \\
6,781 \\
8,476 \\
85,089 \\
12,773 \\
13,872 \\
10,903 \\
179,917\end{array}$ & $\begin{array}{r}11,856 \\
26,782 \\
41 \\
3,297 \\
5,992 \\
10,247 \\
13,865 \\
172,530 \\
10,162 \\
21,143 \\
14,450 \\
290,365\end{array}$ \\
\hline All species & 145,900 & 193,600 & 348,896 & 460,307 \\
\hline
\end{tabular}

$1 /$ Removals in 1976 are trend-level removals. "Other" removals from the transfer of commercial forest 1 and to productive-reserved are not included in 1976 removals.

2/Figures have been adjusted from those published after the 1962 survey to conform to 1976 volumes because of changes in survey definitions and procedures.

3/International $1 / 4-$ inch rule. 


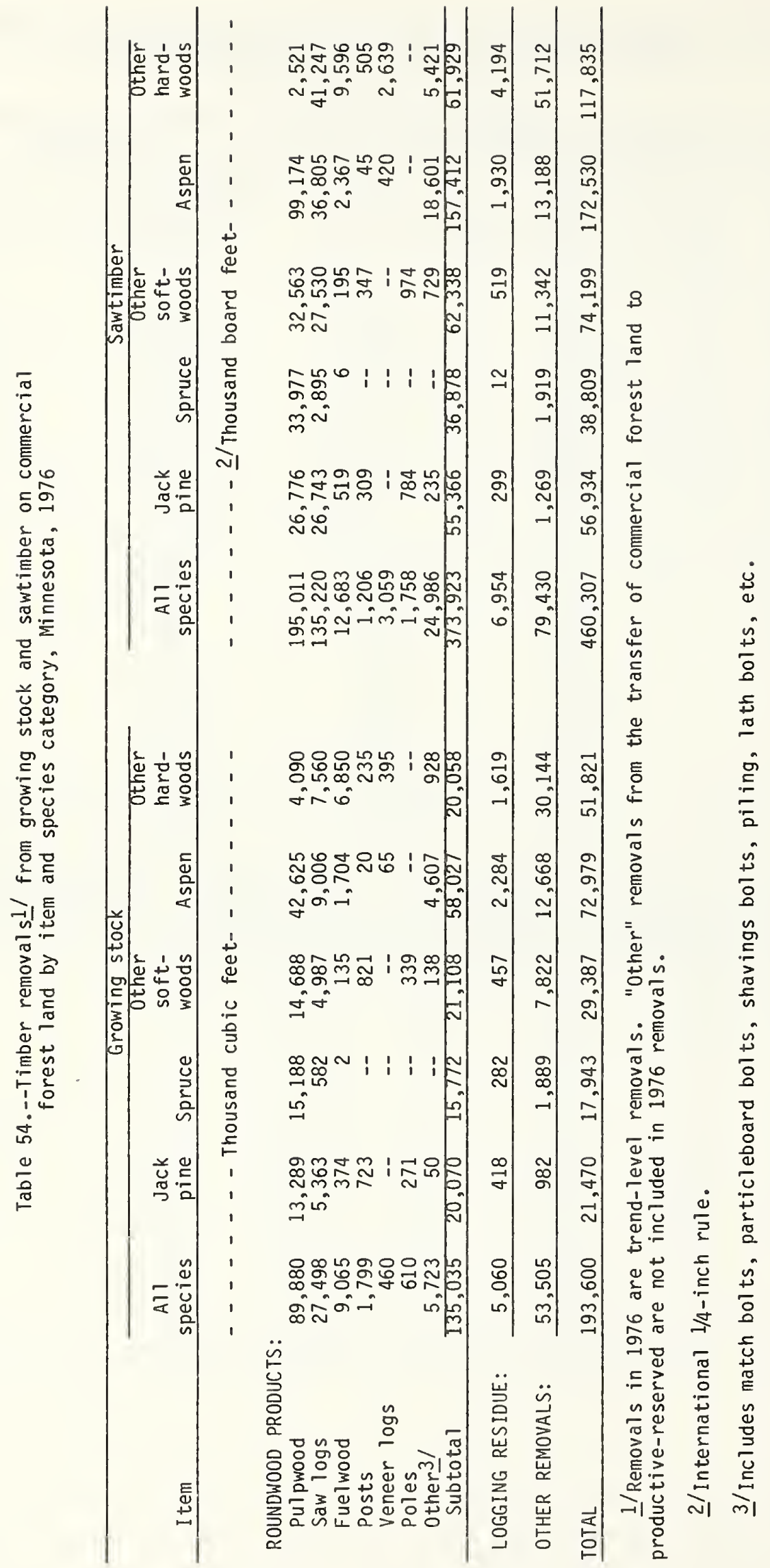


Table 55.--Timber removals 1/ from growing stock on commercial forest 1 and by species group and Forest Survey Unit, Minnesota, 1976

(In thousand cubic feet)

\begin{tabular}{|c|c|c|c|c|c|}
\hline Species & $\begin{array}{l}\text { AाT } \\
\text { units }\end{array}$ & $\begin{array}{l}\text { Aspen- } \\
\text { Birch } \\
\end{array}$ & $\begin{array}{c}\text { Northern } \\
\text { Pine } \\
\end{array}$ & $\begin{array}{l}\text { Central } \\
\text { Hardwood }\end{array}$ & Prairie \\
\hline \multicolumn{6}{|l|}{ SOFTWOODS: } \\
\hline White pine & 2,910 & 1,711 & 1,013 & 186 & -- \\
\hline Red pine & 4,177 & 1,485 & 2,540 & 132 & 20 \\
\hline Jack pine & 21,470 & 10,718 & 10,259 & 484 & 9 \\
\hline White spruce & 4,188 & 2,888 & 1,265 & 20 & 15 \\
\hline Bl ack spruce & 13,755 & 10,275 & 3,458 & 21 & 1 \\
\hline Balsam fir & 13,893 & 7,728 & 6,040 & 119 & 6 \\
\hline Tamarack & 4,982 & 2,503 & 2,126 & 304 & 49 \\
\hline Northern white-cedar & 3,315 & 1,495 & 1,801 & 1 & 18 \\
\hline Other softwoods & 110 & - & - & 72 & 38 \\
\hline Total & 68,800 & 38,803 & 28,502 & 1,339 & 156 \\
\hline \multicolumn{6}{|l|}{ HARDWOODS: } \\
\hline Select white oak & 5,119 & 10 & 595 & 3,136 & 1,378 \\
\hline Select red oàk & 9,779 & 113 & 1,980 & 7,255 & 431 \\
\hline Other red oak & 323 & -- & 8 & 299 & 16 \\
\hline Hickory & 198 & -- & 5 & 182 & 11 \\
\hline Yellow birch & 16 & 3 & 8 & 5 & -- \\
\hline Hard maple & 1,388 & 28 & 319 & 813 & 228 \\
\hline Soft maple & 2,840 & 77 & 269 & 2,356 & 138 \\
\hline Ash & 5,063 & 302 & 1,237 & 2,893 & 631 \\
\hline Balsam poplar & 3,078 & 1,044 & 1,455 & 207 & 372 \\
\hline Paper birch & 9,113 & 3,253 & 4,540 & 1,265 & 55 \\
\hline Bigtooth aspen & 3,444 & 665 & 2,202 & 577 & -- \\
\hline Quaking aspen & 69,535 & 32,686 & 30,440 & 4,965 & 1,444 \\
\hline Basswood & 3,529 & 48 & 805 & 2,000 & 676 \\
\hline Elm & 7,813 & 207 & 1,050 & 4,620 & 1,936 \\
\hline Select hardwoods & 66 & -- & -- & 54 & 12 \\
\hline other hardwoods & 3,496 & 9 & 13 & 2,231 & 1,243 \\
\hline Noncommercial species & & -- & -- & -- & -- \\
\hline Total & 124,800 & 38,445 & 44,926 & 32,858 & 8,571 \\
\hline All species & 193,600 & 77,248 & 73,428 & 34,197 & 8,727 \\
\hline
\end{tabular}

1 Removals in 1976 are trend-level removals. "0ther" removals from the transfer of commercial forest 1 and to productive-reserved are not included in 1976 removals. 
Table 56.--Timber removal 1 / from sawtimber on commercial forest land by species group and Forest Survey Unit, Minnesota, 1976

(In thousand board feet)?

\begin{tabular}{|c|c|c|c|c|c|}
\hline Species & $\begin{array}{l}\text { ATा } \\
\text { units }\end{array}$ & $\begin{array}{l}\text { Aspen- } \\
\text { Birch }\end{array}$ & $\begin{array}{l}\text { Northern } \\
\text { Pine }\end{array}$ & $\begin{array}{l}\text { Central } \\
\text { Hardwood }\end{array}$ & Prairie \\
\hline \multicolumn{6}{|l|}{ SOFTWOODS: } \\
\hline White pine & 13,190 & 7,641 & 5,026 & 523 & -- \\
\hline Red pine & 15,214 & 4,210 & 10,535 & 405 & 64 \\
\hline Jack pine & 56,934 & 27,678 & 27,943 & 1,296 & 17 \\
\hline White spruce & 12,679 & 6,368 & 6,224 & 54 & 33 \\
\hline Black spruce & 26,130 & 21,857 & 4,269 & 4 & -- \\
\hline Balsam fir & 27,000 & 15,329 & 11,563 & 108 & -- \\
\hline Tamarack & 10,165 & 5,237 & 4,523 & 318 & 87 \\
\hline Northern white-cedar & 8,548 & 4,948 & 3,568 & 8 & 24 \\
\hline Other softwoods & 82 & -- & -- & 50 & 32 \\
\hline Total & 169,942 & 93,268 & 73,651 & 2,766 & $25 T$ \\
\hline \multicolumn{6}{|l|}{ HARDWOODS: } \\
\hline Select white oak & 11,856 & 10 & 1,729 & 7,494 & 2,623 \\
\hline Select red oak & 25,721 & 160 & 4,587 & 20,026 & 948 \\
\hline other red oak & 1,061 & -- & 10 & 1,010 & 41 \\
\hline Hickory & 274 & -- & 9 & 252 & 13 \\
\hline Yellow birch & 41 & 14 & 17 & 10 & -- \\
\hline Hard maple & 3,297 & 39 & 676 & 2,103 & 479 \\
\hline Soft maple & 5,992 & 69 & 558 & 4,971 & 394 \\
\hline Ash & 10,247 & 683 & 3,353 & 4,861 & 1,350 \\
\hline Balsam poplar & 4,508 & 1,165 & 2,522 & 603 & 218 \\
\hline Paper birch & 13,865 & 6,582 & 6,257 & 1,012 & 14 \\
\hline Bigtooth aspen & 8,797 & 1,472 & 5,473 & 1,852 & -- \\
\hline Quaking aspen & 163,733 & 73,787 & 76,701 & 12,708 & 537 \\
\hline Basswood & 10,162 & 152 & 2,768 & 5,353 & 1,889 \\
\hline Elm & 21,143 & 618 & 4,073 & 11,371 & 5,081 \\
\hline Select hardwoods & 374 & -- & -- & 298 & 76 \\
\hline other hardwoods & 9,294 & 7 & 22 & 5,312 & 3,953 \\
\hline Noncommercial species & $-\infty$ & -- & -- & $-\infty$ & -- \\
\hline Total & 290,365 & 84,758 & 108,755 & 79,236 & 17,616 \\
\hline All species & 460,307 & 178,026 & 182,406 & 82,002 & 17,873 \\
\hline
\end{tabular}

1 Removals in 1976 are trend-level removals. "Other" removals from the transfer of commercial forest 1 and to productive-reserved are not included in 1976 removals.

2/International $1 / 4$-inch rule. 
Table 57.--Net annual growth and removals $1 /$ of growing stock on commercial forest land by species group, Minnesota, 1976

(In thousand cubic feet)

\begin{tabular}{|c|c|c|}
\hline Species & $\begin{array}{l}\text { Net annual } \\
\text { growth }\end{array}$ & $\begin{array}{c}\text { Annual timber } \\
\text { removals }\end{array}$ \\
\hline \multicolumn{3}{|l|}{ SOFTWOODS: } \\
\hline White pine & 5,599 & 2,910 \\
\hline Red pine & 15,684 & 4,177 \\
\hline Jack pine & 25,677 & 21,470 \\
\hline White spruce & 10,701 & 4,188 \\
\hline Black spruce & 16,520 & 13,755 \\
\hline Balsam fir & 31,902 & 13,893 \\
\hline Tamarack & 2,269 & 4,982 \\
\hline Northern white-cedar & 11,274 & 3,315 \\
\hline Other softwoods & 155 & 110 \\
\hline Total & 119,781 & 68,800 \\
\hline \multicolumn{3}{|l|}{ HARDWOODS: } \\
\hline Select white oak & 8,733 & 5,119 \\
\hline Select red oak & 15,691 & 9,779 \\
\hline Other red oak & 256 & 323 \\
\hline Hickory & 455 & 198 \\
\hline Yellow birch & 118 & 16 \\
\hline Hard maple & 8,660 & 1,388 \\
\hline Soft maple & 6,454 & 2,840 \\
\hline Ash & 9,468 & 5,063 \\
\hline Balsam poplar & 17,757 & 3,078 \\
\hline Paper birch & 30,290 & 9,113 \\
\hline Bigtooth aspen & 1,086 & 3,444 \\
\hline Quaking aspen & 93,083 & 69,535 \\
\hline Basswood & 17,252 & 3,529 \\
\hline E $7 m$ & 18,022 & 7,813 \\
\hline Select hardwoods & 440 & 66 \\
\hline Other hardwoods & 1,374 & 3,496 \\
\hline Noncommercial species & -- & -- \\
\hline Total & 229,139 & 124,800 \\
\hline All species & 348,920 & 193,600 \\
\hline
\end{tabular}

1/Removals in 1976 are trend-level removals. "Other" removals from the transfer of commercial forest land to productivereserved are not included in 1976 removals. 
Table 58.--Net annual growth and removals $1 /$ of sawtimber on commercial forest land by species group, Minnesota, 1976

(In thousand board feet)ㄹㄱ

\begin{tabular}{|c|c|c|}
\hline Species & $\begin{array}{l}\text { Net annua } 7 \\
\text { growth }\end{array}$ & $\begin{array}{c}\text { Annual timber } \\
\text { removals }\end{array}$ \\
\hline \multicolumn{3}{|l|}{ SOFTWOODS: } \\
\hline White pine & 33,304 & 13,190 \\
\hline Red pine & 74,768 & 15,214 \\
\hline Jack pine & 88,650 & 56,934 \\
\hline White spruce & 36,750 & 12,679 \\
\hline Black spruce & 18,568 & 26,130 \\
\hline Balsam fir & 86,053 & 27,000 \\
\hline Tamarack & 8,460 & 10,165 \\
\hline Northern white-cedar & 33,003 & 8,548 \\
\hline Other softwoods & 763 & 82 \\
\hline Total & 380,319 & 169,942 \\
\hline \multicolumn{3}{|l|}{ HARDWOODS: } \\
\hline Select white oak & 30,845 & 11,856 \\
\hline Select red oak & 61,191 & 25,721 \\
\hline Other red oak & 960 & 1,061 \\
\hline Hickory & 1,484 & 274 \\
\hline Yellow birch & 612 & 41 \\
\hline Hard maple & 30,649 & 3,297 \\
\hline Soft maple & 18,380 & 5,992 \\
\hline Ash & 18,003 & 10,247 \\
\hline Balsam poplar & 51,922 & 4,508 \\
\hline Paper birch & 53,434 & 13,865 \\
\hline Bigtooth aspen & 11,116 & 8,797 \\
\hline Quaking aspen & 330,071 & 163,733 \\
\hline Basswood & 48,788 & 10,162 \\
\hline $\begin{array}{l}\text { Elm } \\
\text { Select hardwoods }\end{array}$ & 61,699 & 21,143 \\
\hline Select hardwoods & 2,261 & 374 \\
\hline Other hardwoods & 9,730 & 9,294 \\
\hline Noncommercial species & -. & -. \\
\hline Total & 731,145 & 290,365 \\
\hline All species & $1,111,464$ & 460,307 \\
\hline \multicolumn{3}{|c|}{$\begin{array}{l}1 / \text { Removals in } 1976 \text { are trend-level removals. "Other" remo } \\
\text { vals from the transfer of commercial forest land to productive } \\
\text { reserved are not included in } 1976 \text { removals. }\end{array}$} \\
\hline national $1 /$ & & \\
\hline
\end{tabular}


Table 59.--Net annual growth and removalsl/ of growing stock on commercial forest land by ownership class and softwoods and hardwoods, Minnesota, 1976

(In thousand cubic feet)

FEDERAL

\begin{tabular}{|c|c|c|c|c|c|c|}
\hline \multirow[b]{2}{*}{ Owner } & \multicolumn{3}{|c|}{ Net annual growth } & \multicolumn{3}{|c|}{ Annual timber removals } \\
\hline & $\begin{array}{c}\text { AाT } \\
\text { species }\end{array}$ & Softwoods & Hardwoods & $\begin{array}{c}\text { AाI } \\
\text { species }\end{array}$ & Sof twoods & Hardwoods \\
\hline $\begin{array}{l}\text { National Forest } \\
\text { Other federal } \\
\text { Subtotal }\end{array}$ & $\begin{array}{l}50,903 \\
18,686 \\
69,589\end{array}$ & $\begin{array}{r}25,111 \\
7,048 \\
32,159\end{array}$ & $\begin{array}{r}25,792 \\
11,638 \\
37,430\end{array}$ & $\begin{array}{r}16,423 \\
5,202 \\
21,625\end{array}$ & $\begin{array}{r}7,946 \\
2,686 \\
10,632 \\
\end{array}$ & $\begin{array}{r}8,477 \\
2,516 \\
10,993\end{array}$ \\
\hline
\end{tabular}

STATE

\begin{tabular}{llllllll}
\hline Total & 63,052 & 25,862 & 37,190 & 34,123 & 18,920 & 15,203 \\
\hline
\end{tabular}

COUNTY \& MUNICIPAL

\begin{tabular}{lrrrrrr}
\hline Total & 54,203 & 23,653 & 30,550 & 18,354 & 5,753 & 12,601 \\
\hline \multicolumn{7}{c}{ PRIVATE } \\
\hline $\begin{array}{c}\text { Forest industry } \\
\text { Farm and other }\end{array}$ & 15,899 & 8,872 & 7,027 & 15,165 & 7,491 & 7,674 \\
$\quad$ Subtotal & 146,177 & 29,235 & 116,942 & 104,333 & 26,004 & 78,329 \\
\hline
\end{tabular}

ALL OWNERS

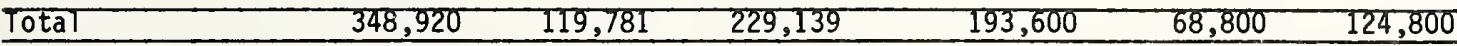

1 Removals in 1976 are trend-level removals. "Other" removals from the transfer of commercial forest 1 and to productive-reserved are not included in 1976 removals.

Table 60.--Net annual growth and removals1/ of sawtimber on commercial forest land by ownership class and softwoods and hardwoods, Minnesota, 1976

(In thousand board feet) 2/

FEDERAL

\begin{tabular}{|c|c|c|c|c|c|c|}
\hline \multirow{3}{*}{$\begin{array}{l}\text { Owner } \\
\text { National forest } \\
\text { Other federal } \\
\text { Subtotal }\end{array}$} & \multicolumn{3}{|c|}{ Net annual growth } & \multicolumn{3}{|c|}{ Annual timber removals } \\
\hline & $\begin{array}{c}\text { AाT } \\
\text { species }\end{array}$ & Sof twoods & Hardwoods & $\begin{array}{c}\text { ATT } \\
\text { species }\end{array}$ & Softwoods & Hardwoods \\
\hline & $\begin{array}{r}208,935 \\
45,034 \\
253,969\end{array}$ & $\begin{array}{r}117,477 \\
15,357 \\
132,834\end{array}$ & $\begin{array}{r}91,458 \\
29,677 \\
121 \\
\end{array}$ & $\begin{array}{r}41,631 \\
12,926 \\
54,557\end{array}$ & $\begin{array}{r}21,862 \\
6,839 \\
28701 \\
\end{array}$ & $\begin{array}{r}19,769 \\
6,087 \\
\end{array}$ \\
\hline \multicolumn{7}{|c|}{ STATE } \\
\hline Total & 151,118 & 58,859 & 92,259 & 90,134 & 52,004 & 38,130 \\
\hline
\end{tabular}

COUNTY \& MUNICIPAL

\begin{tabular}{ccccccc}
\hline Total & 182,828 & 66,220 & 116,608 & 46,436 & 14,769 & 31,667 \\
\hline & PR IVATE
\end{tabular}

\begin{tabular}{lrrrrrr}
\hline Forest industry & 60,338 & 27,906 & 32,432 & 34,606 & 16,845 & 17,761 \\
Farmer and other & 463,211 & 94,500 & 368,711 & 234,574, & 57,623 & 176,951 \\
\cline { 2 - 7 } & 523,549 & 122,406 & 401,143 & 269,180 & 74,468 & 194,712 \\
\hline
\end{tabular}

ALL OWNERS

\begin{tabular}{llllllll}
\hline Total & $1,111,464$ & 380,319 & 731,145 & 460,307 & 169,942 & 290,365 \\
\hline
\end{tabular}

1/Removals in 1976 are trend-level removals. "Other" removals from the transfer of commercial forest 1 and to productive-reserved are not included in 1976 removals.

2/International 1/4-inch rule. 
Table 61.--Annual mortality of growing stock and sawtimber on commercial forest land by species group, Minnesota, 1976

\begin{tabular}{|c|c|c|}
\hline Species & Growing stock & Sawtimber \\
\hline & $\begin{array}{l}\text { Thousand } \\
\text { cubic feet }\end{array}$ & $\begin{array}{l}\text { Thousand } \\
\text { board feet } 1 /\end{array}$ \\
\hline \multicolumn{3}{|l|}{ SOF TWOODS: } \\
\hline White pine & 960 & 2,994 \\
\hline Red pine & 110 & 345 \\
\hline Jack pine & 3,322 & 6,009 \\
\hline White spruce & 1,102 & 3,408 \\
\hline Black spruce & 3,899 & 1,513 \\
\hline Balsam fir & 16,774 & 21,233 \\
\hline Tamarack & 5,857 & 8,748 \\
\hline Northern white-cedar & 1,704 & 6,212 \\
\hline Other & -- & - \\
\hline Total & 33,728 & 50,462 \\
\hline \multicolumn{3}{|l|}{ HARDWOODS: } \\
\hline Select white oak & $\begin{array}{l}1,098 \\
2,093\end{array}$ & $\begin{array}{r}3,444 \\
10,815\end{array}$ \\
\hline $\begin{array}{l}\text { Select red oak } \\
\text { Other red oak }\end{array}$ & $\begin{array}{r}2,993 \\
--\end{array}$ & 10,810 \\
\hline Hickory & -- & -- \\
\hline Yellow birch & 87 & 27 \\
\hline Hard maple & 1,122 & 1,003 \\
\hline Soft maple & 252 & 914 \\
\hline Ash & 5,071 & 11,195 \\
\hline Bal sam poplar & 10,723 & 17,656 \\
\hline Paper birch & 4,870 & 7,231 \\
\hline Bigtooth aspen & 4,841 & 7,153 \\
\hline Quaking aspen & 66,519 & 110,093 \\
\hline Basswood & 1,410 & 5,043 \\
\hline Elm & 8,125 & 37,957 \\
\hline Select hardwoods & 223 & -- \\
\hline Other hardwoods & 479 & -- \\
\hline Noncommercial species & -- & -- \\
\hline Total & 107,813 & 212,531 \\
\hline All species & 141,541 & 262,993 \\
\hline
\end{tabular}

$1 /$ International $1 / 4$-inch rule. 


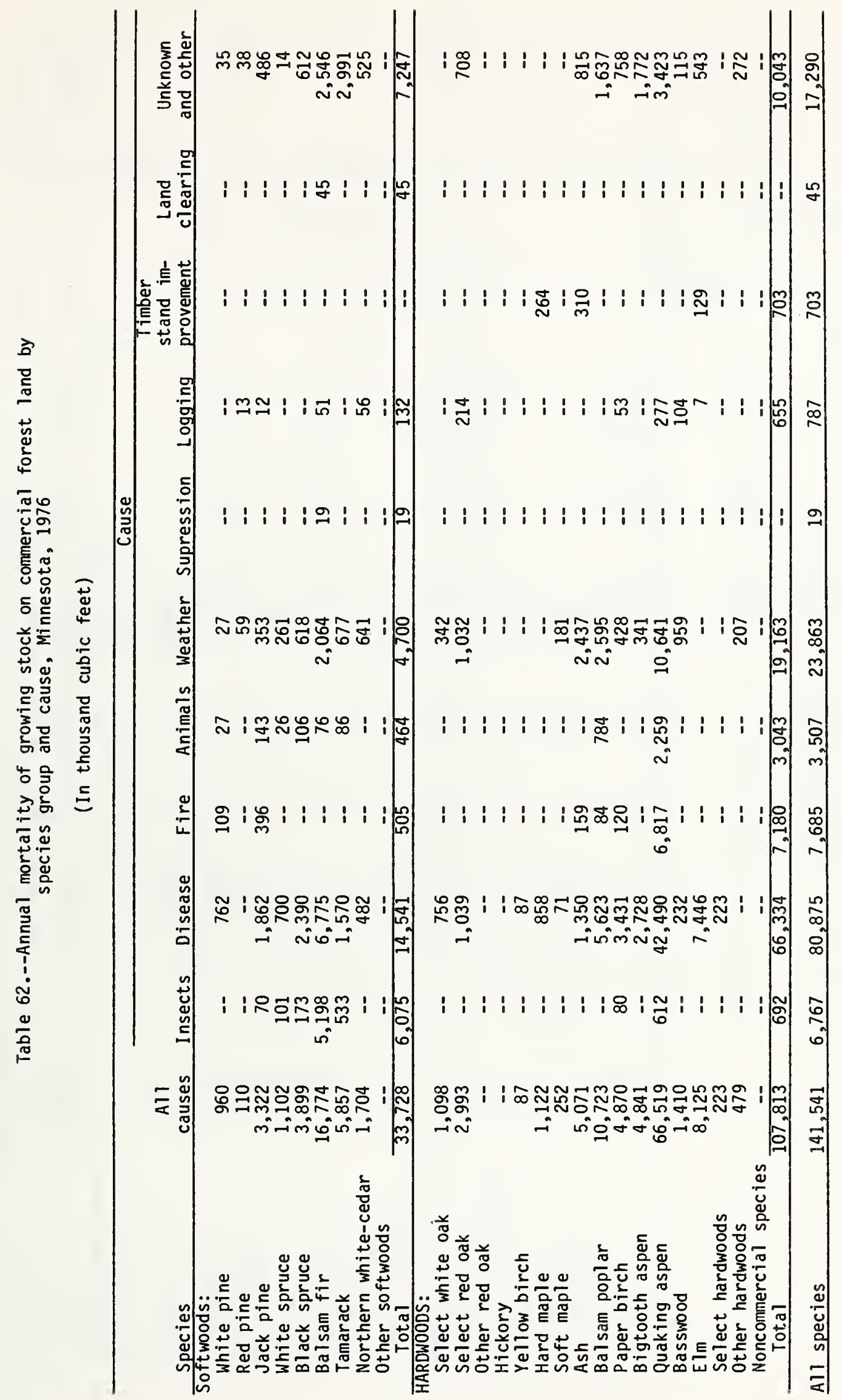




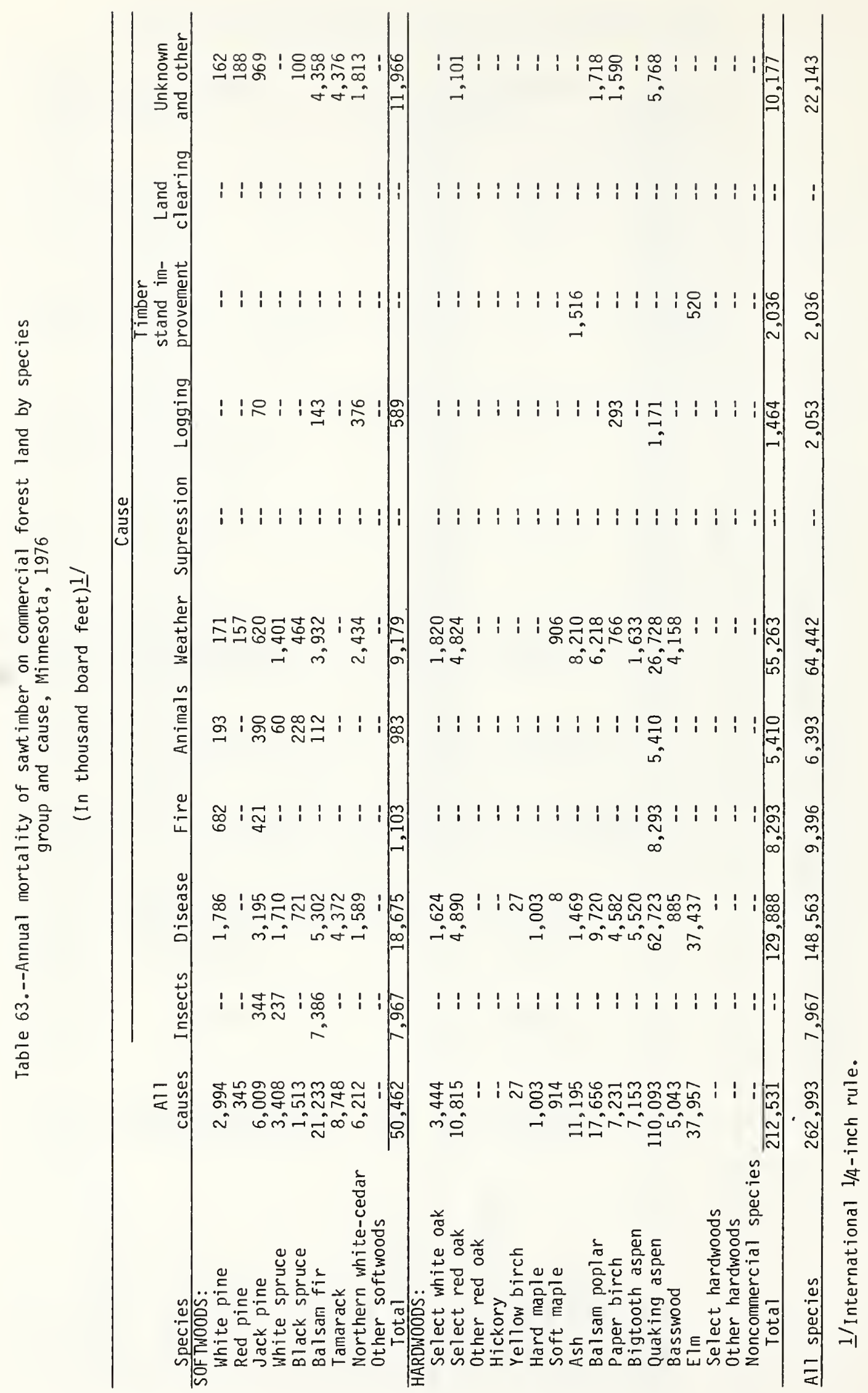


Table 64.--Annual mortality of growing stock and sawtimber on commercial forest land by ownership class and softwoods and hardwoods, Minnesota, 1976

\begin{tabular}{|c|c|c|c|c|c|c|}
\hline \multirow[b]{2}{*}{ Owner } & \multicolumn{3}{|c|}{ Growing stock } & \multicolumn{3}{|c|}{ Sawtimber } \\
\hline & $\begin{array}{l}\text { ATा } \\
\text { species }\end{array}$ & Softwoods & Hardwoods & $\begin{array}{c}\text { ATT } \\
\text { species }\end{array}$ & Softwoods & Hardwoods \\
\hline & --- & sand cubi & $t--\cdot$ & $--\frac{1}{1}$ & usand boarc & et- - - - \\
\hline National Forest & 14,272 & 7,505 & 6,767 & 9,352 & 2,426 & 6,926 \\
\hline Bureau of Land Management & -1 & -- & & 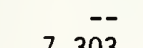 & -- & -- \\
\hline $\begin{array}{l}\text { Indian } \\
\text { Miscellaneous federal } \\
\text { State } \\
\text { County and municipal } \\
\text { Forest industry } \\
\text { Farmer }\end{array}$ & $\begin{array}{r}4,029 \\
861 \\
20,998 \\
31,990 \\
12,680 \\
36,797\end{array}$ & $\begin{array}{r}1,019 \\
131 \\
8,772 \\
4,477 \\
3,534 \\
4,312\end{array}$ & $\begin{array}{r}3,010 \\
730 \\
12,226 \\
27,513 \\
9,146 \\
32,485\end{array}$ & $\begin{array}{r}7,303 \\
1,914 \\
35,131 \\
51,818 \\
18,497 \\
94,273\end{array}$ & $\begin{array}{r}1,994 \\
388 \\
12,350 \\
7,623 \\
6,419 \\
10,728\end{array}$ & $\begin{array}{r}5,309 \\
1,526 \\
22,781 \\
44,195 \\
12,078 \\
83,545\end{array}$ \\
\hline Farmer owned-leased & & -- & -- & -- & -- & -- \\
\hline $\begin{array}{l}\text { Misc. private-corp. } \\
\text { Misc. private-indiv. }\end{array}$ & $\begin{array}{r}6,213 \\
13,507\end{array}$ & $\begin{array}{l}1,543 \\
2,435\end{array}$ & $\begin{array}{r}4,670 \\
11,072\end{array}$ & $\begin{array}{l}16,586 \\
28,119\end{array}$ & $\begin{array}{l}3,994 \\
4,540\end{array}$ & $\begin{array}{l}12,592 \\
23,579\end{array}$ \\
\hline Misc. priv.-corp., leased & 194 & - & 194 & - & - & -- \\
\hline Misc. priv.-indiv., leased & $\cdots$ & - & - & -- & - & -- \\
\hline 11 owners & 141,541 & 33,728 & 107,813 & 262,993 & 50,462 & 212,531 \\
\hline
\end{tabular}

$1 /$ International $1 / 4$-inch rule. 


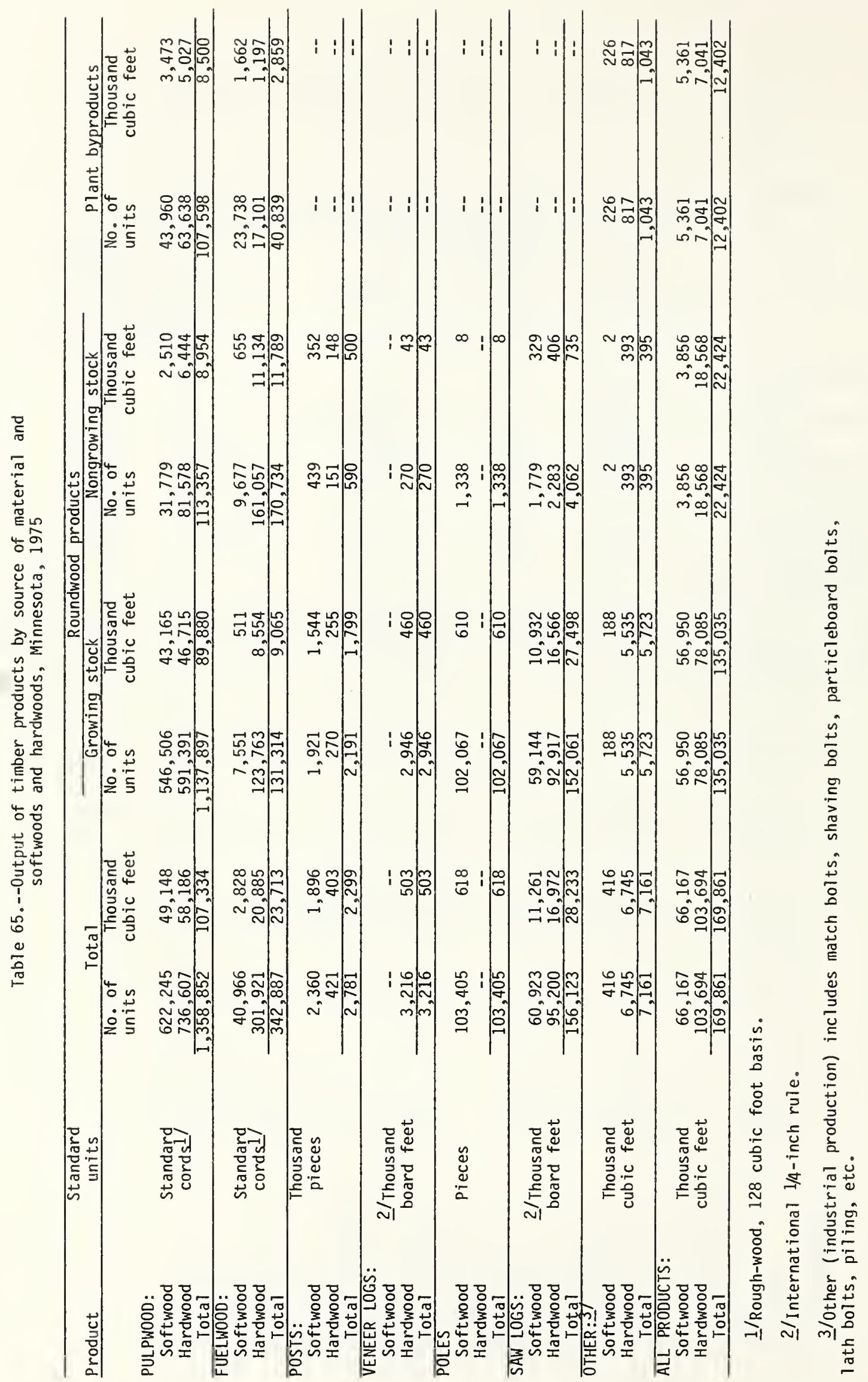




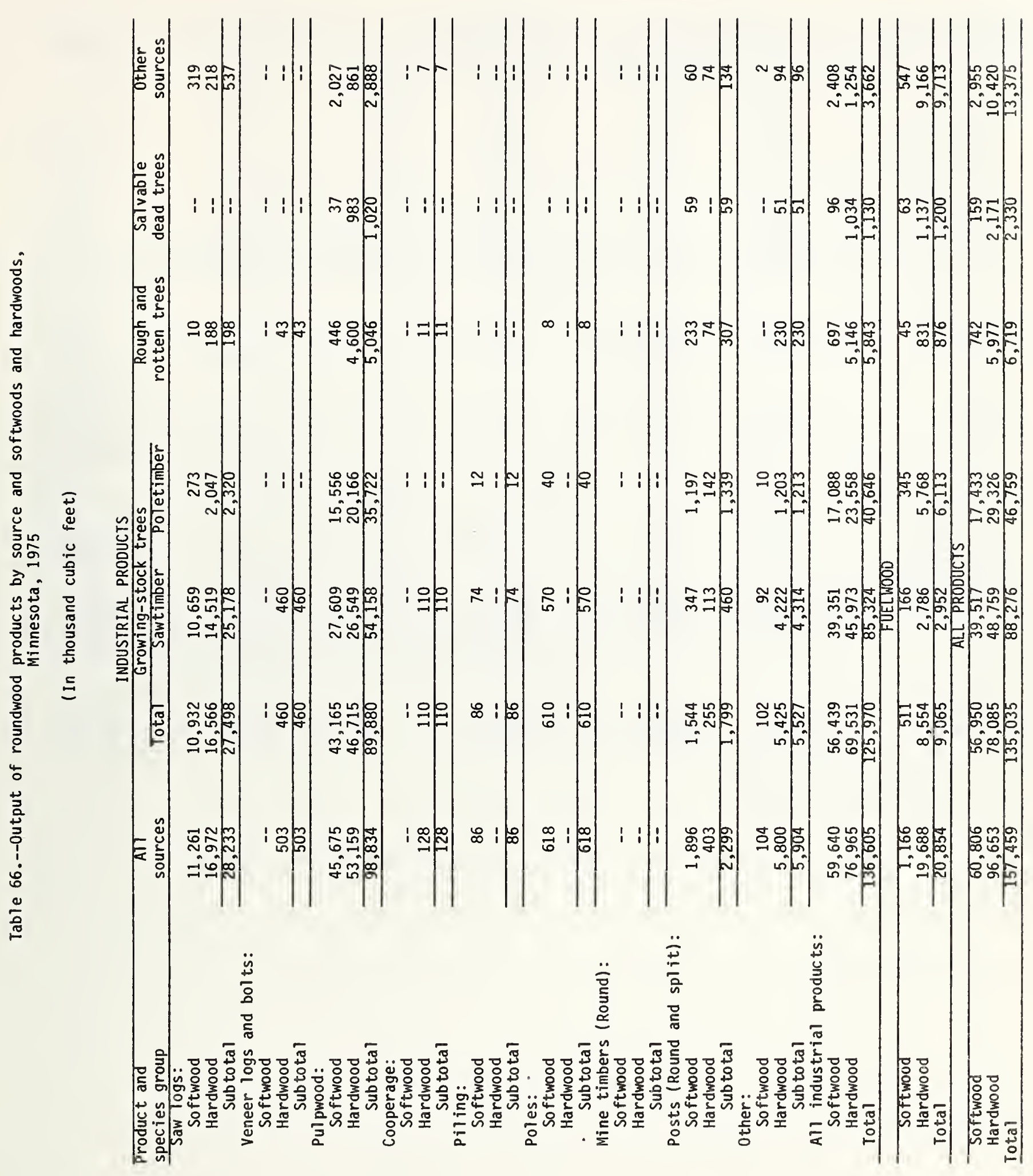


Table 67.--Forest products harvested by ownership class and product, Minnesota, 1975

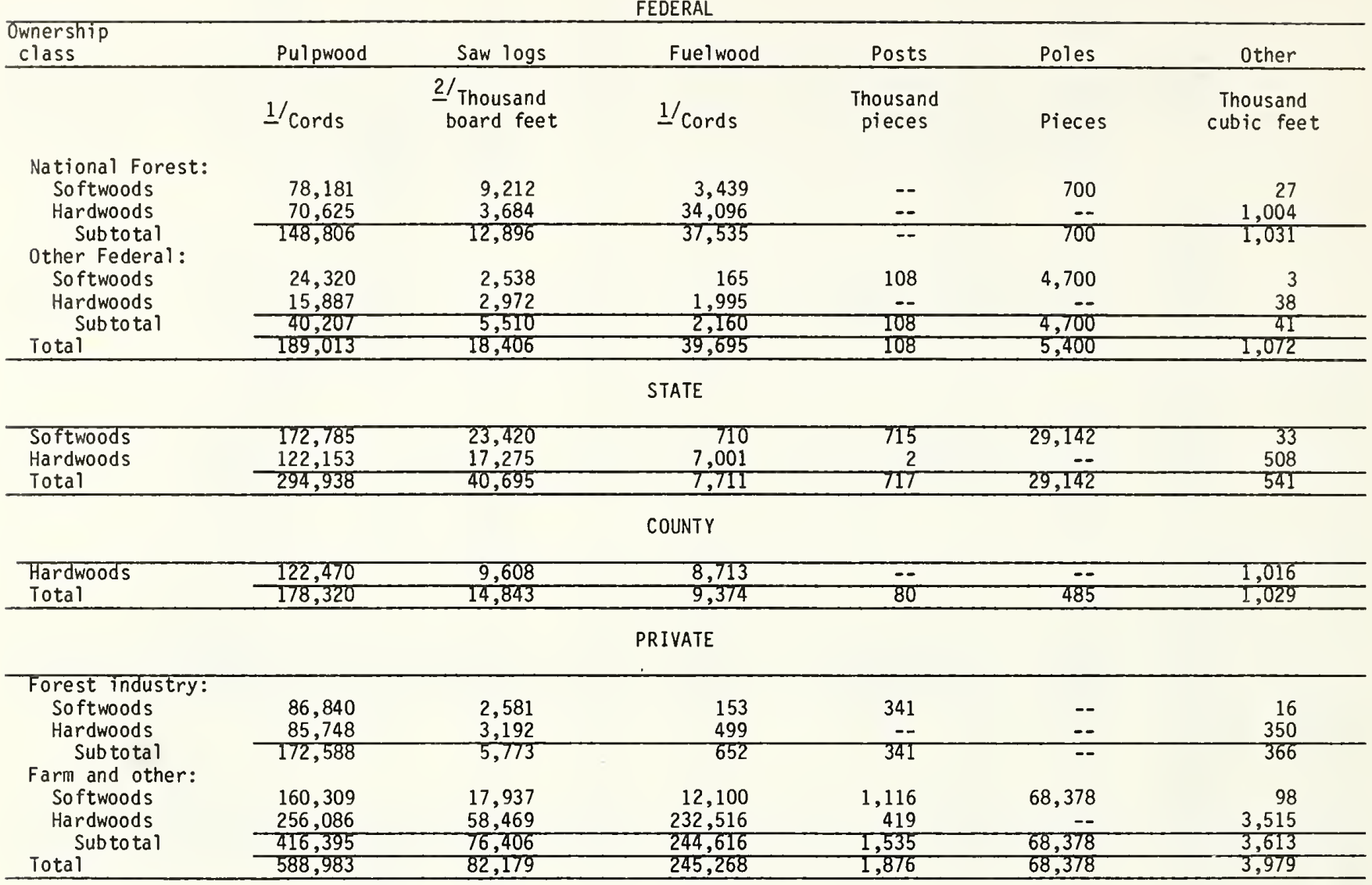

ALL OWNERS

\begin{tabular}{lrrrrrr}
\hline Softwoods & 578,285 & 60,923 & 17,228 & 2,360 & 103,405 & -- \\
Hardwoods & 672,969 & 95,200 & 284,820 & 421 & 6,431 \\
Total & $1,251,254$ & 156,123 & 302,048 & 2,781 & 103,405 \\
\hline
\end{tabular}

$1 /$ Rough-wood, 128 cubic foot basis.

2/International $1 / 4$-inch rule. 
Table 68.--Timber products from roundwood by species group and product, Minnesota, 1975

\begin{tabular}{|c|c|c|c|c|c|c|c|}
\hline \multirow[t]{2}{*}{ Species } & \multirow{2}{*}{$\begin{array}{l}\text { 1/Thousand } \\
\text { Products } \\
\begin{array}{l}\text { cub ic } \\
\text { feet }\end{array}\end{array}$} & \multicolumn{2}{|c|}{ Saw logs } & \multicolumn{2}{|c|}{ Veneer logs } & \multicolumn{2}{|c|}{ Pulpwood } \\
\hline & & $\begin{array}{l}\text { 2/Thousand } \\
\text { board } \\
\text { feet }\end{array}$ & $\begin{array}{c}1 / \text { Thousand } \\
\text { cubic } \\
\text { feet }\end{array}$ & $\begin{array}{l}\text { 2/Thousand } \\
\text { board } \\
\text { feet }\end{array}$ & $\begin{array}{l}\text { Thousand } \\
\text { cubic } \\
\text { feet }\end{array}$ & $\begin{array}{l}\text { Thousand } \\
\text { cords }\end{array}$ & $\begin{array}{l}1 / \text { Thousand } \\
\text { cubic } \\
\text { feet }\end{array}$ \\
\hline \multicolumn{8}{|l|}{ SOF TWOODS: } \\
\hline White pine & 2,541 & 11,272 & 1,943 & -- & -- & 7,386 & 584 \\
\hline Red pine & 3,519 & 10,680 & 1,840 & -- & -- & 10,790 & 850 \\
\hline Jack pine & 21,922 & 29,058 & 5,579 & -- & -- & 180,543 & 14,260 \\
\hline Spruce & 16566 & 3,145 & 605 & -- & -- & 202,004 & 15,956 \\
\hline Balsam fir & 10,073 & 2,100 & 400 & -- & -- & 121,296 & 9,583 \\
\hline Tamarack & 4,620 & & 14 & -- & -- & 56,266 & 4,442 \\
\hline Northern white-cedar & 1,565 & 4,589 & 880 & -- & -- & -- & -- \\
\hline Total & 60,806 & 60,923 & 11,261 & -- & -- & 578,285 & 45,675 \\
\hline \multicolumn{8}{|l|}{ HARDWOOUS: } \\
\hline Whi te oak & 2,498 & 4,187 & 695 & 29 & 3 & -- & -- \\
\hline Red oak & 7,917 & 14,355 & 2,404 & 751 & 118 & 9,703 & 769 \\
\hline Hickory & 29 & 78 & 13 & -- & -- & -- & -- \\
\hline Yellow birch & 23 & -- & -- & 20 & 3 & 3 & -- \\
\hline Hard maple & 747 & 934 & 153 & 290 & 45 & 200 & 13 \\
\hline Soft maple & 546 & 781 & 130 & 168 & 25 & 79 & 3 \\
\hline Ash & 2,639 & 3,954 & 683 & 17 & 2 & 55 & 5 \\
\hline Bal sam poplar & 1,210 & 1,269 & 222 & -- & - & 10,725 & 848 \\
\hline Paper birch & 8,294 & 2,728 & 473 & 190 & 29 & 35,474 & 2,804 \\
\hline Aspen & 66,831 & 49,501 & 9,166 & 443 & 72 & 615,995 & 48,660 \\
\hline Basswood & 1,042 & 4,369 & 758 & 417 & 66 & -- & -- \\
\hline Elm & 3,810 & 9,508 & 1,665 & 376 & 59 & 733 & 57 \\
\hline Black walnut & 61 & 236 & 37 & 151 & 24 & -- & -- \\
\hline Cottonwood & 778 & 3,196 & 556 & 358 & 56 & -- & -- \\
\hline Other hardwoods & 228 & 104 & 17 & 6 & 1 & 2 & -- \\
\hline Total & 96,653 & 95,200 & 16,972 & 3,216 & 503 & 672,969 & 53,159 \\
\hline All species & 157,459 & 156,123 & 28,233 & 3,216 & 503 & $1,251,254$ & 98,834 \\
\hline
\end{tabular}

$1 /$ Small quantities may round off to less than 500 cubic feet and will be shown as a dash in columns showing thousand cubic feet.

2/International 1/4-inch rule. 


\begin{tabular}{|c|c|c|c|c|c|c|c|}
\hline \multirow[t]{2}{*}{ Species } & \multicolumn{2}{|r|}{ Fuel wood } & \multicolumn{2}{|c|}{ Poles } & \multicolumn{2}{|c|}{ Posts } & \multirow{2}{*}{$\begin{array}{l}\text { Other } \\
\text { Products } \\
\text { 1/Thousand } \\
\text { cubic } \\
\text { feet }\end{array}$} \\
\hline & Cords & $\begin{array}{c}\text { 1/Thousand } \\
\text { cubic } \\
\text { feet }\end{array}$ & Pieces & $\begin{array}{c}1 / \text { Thousand } \\
\text { cubic } \\
\text { feet }\end{array}$ & $\begin{array}{l}\text { Thousand } \\
\text { pieces }\end{array}$ & $\begin{array}{c}1 / \text { Thousand } \\
\text { cub ic } \\
\text { feet }\end{array}$ & \\
\hline SOF TWOODS: & & & & & & & \\
\hline White pine & 360 & 14 & -- & -- & -- & -- & -- \\
\hline Red pine & & 27 & 57,284 & 343 & 523 & 419 & 40 \\
\hline Jack pine & 12,353 & 865 & 46,121 & 275 & 1110 & 893 & 50 \\
\hline Spruce & 125 & 5 & - & - & - & -- & -- \\
\hline Bal sam fir & 1,338 & 90 & -- & -- & -- & -- & -- \\
\hline Tamarack & 1,919 & 126 & -- & -- & 49 & 38 & -- \\
\hline Northern whi te-cedar & 614 & 39 & -- & -- & 678 & 546 & 100 \\
\hline Total & 17,228 & 1,166 & 103,405 & 618 & 2,360 & 1,896 & 190 \\
\hline HARDWOODS: & & & & & & & \\
\hline $\begin{array}{l}\text { Whi te oak } \\
\text { Red oak }\end{array}$ & $\begin{array}{l}21,759 \\
63,515\end{array}$ & $\begin{array}{l}1,509 \\
4,436\end{array}$ & -- & -- & $\begin{array}{l}233 \\
139\end{array}$ & 231 & 60 \\
\hline Hickory & 410 & 16 & -- & -- & 139 & $\begin{array}{l}140 \\
--\end{array}$ & 50 \\
\hline Yellow birch & 364 & 20 & -- & -- & -- & -- & -- \\
\hline Hard maple & 7,820 & 521 & -- & -- & -- & -- & 15 \\
\hline Soft maple & 5,805 & 388 & -- & -- & -- & -- & -- \\
\hline Ash & 28,206 & 1,945 & -- & -- & 11 & 4 & -- \\
\hline Bal sam poplar & 2,101 & 140 & -- & -- & 3 & -- & -- \\
\hline Paper birch & 61,335 & 4,276 & -- & -- & - & -- & 712 \\
\hline Aspen & 56,326 & 3,937 & -- & -- & 35 & 28 & 4,968 \\
\hline Basswood & 2,652 & 149 & -- & -- & -- & -- & 69 \\
\hline Elm & 28,280 & 1,975 & -- & -- & -- & -- & 54 \\
\hline Black walnut & - & -- & -- & -- & -- & -- & - \\
\hline Cottonwood & 2,811 & 166 & -- & -- & -- & -- & -- \\
\hline Other hardwoods & 3,436 & 210 & -- & -- & -- & -- & -- \\
\hline Tota 1 & 284,820 & 19,688 & -- & -- & 421 & 403 & 5,928 \\
\hline All species & 302,048 & 20,854 & 103,405 & 618 & 2,781 & 2,299 & 6,118 \\
\hline
\end{tabular}


Table 69.--Volume of primary plant residue by kind of material and type of use, Minnesota, 1975

(In thousand cubic feet)

Kind of wood residue

\begin{tabular}{|c|c|c|c|c|c|c|c|c|}
\hline \multirow{3}{*}{$\begin{array}{l}\text { Type } \\
\text { of use } \\
\end{array}$} & \multicolumn{6}{|c|}{ Kind of wood residue } & & \\
\hline & \multicolumn{2}{|c|}{ Total } & \multicolumn{2}{|c|}{ Coarse $1 /$} & \multicolumn{2}{|c|}{$\mathrm{Fine}^{2 /}$} & \multicolumn{2}{|c|}{ Bark $\underline{3}$ / } \\
\hline & Softwood & Hardwood & Softwood & Hardwood & Softwood & Hardwood & Softwood & Hardwood \\
\hline Fiber products 4 / & $1,127.1$ & $2,968.2$ & $1,015.1$ & $2,797.1$ & 112.0 & 171.1 & -- & 76.6 \\
\hline Charcoal & 57.6 & 216.2 & 57.6 & 216.2 & -- & -- & 35.9 & 100.2 \\
\hline Industrial fuel & $1,292.9$ & 161.4 & 800.7 & 28.1 & 492.2 & 133.3 & 694.1 & 404.5 \\
\hline Domestic fuel & 368.4 & $1,034.9$ & 354.4 & 851.5 & 14.0 & 183.4 & 167.8 & 423.5 \\
\hline Miscell aneous $\underline{5}$ & 167.9 & 600.9 & 89.7 & 51.1 & 78.2 & 549.8 & 14.0 & 459.2 \\
\hline Not used- & $\underline{1,430.0}$ & $2,155.8$ & 494.0 & 508.1 & 936.0 & $1,647.7$ & $1,109.7$ & $2,136.1$ \\
\hline Total & $4,443.9$ & $7,137.4$ & $2,811.5$ & $4,452.1$ & $1,632.4$ & $2,685.3$ & $2,021.5$ & $3,600.1$ \\
\hline
\end{tabular}

$\underline{1}$ Suitable for chipping such as slabs, edgings, veneer cores, etc.

2/Not suitable for chipping such as sawdust, veneer clippings, etc.

3/Does not include bark disposal at pulpmills.

$4 /$ For manufacture of pulp, hardboard or roofing felt.

5/Livestock bedding, mulch, small dimension, and specialty items.

6/Includes residue burned as waste.

Table 70.--Sampling errors- for estimates smaller than the State totals of volume, net growth, removals, and area of commercial forest land, Minnesota, 1977

\begin{tabular}{|c|c|c|c|c|c|c|c|}
\hline \multirow{2}{*}{$\begin{array}{l}\text { Sampl ing } \\
\text { error }\end{array}$} & \multirow{2}{*}{$\begin{array}{c}\text { Commercial } \\
\text { forest } \\
\text { area }\end{array}$} & \multicolumn{3}{|c|}{ Growing Stock } & \multicolumn{3}{|c|}{ Sawtimber } \\
\hline & & Inventory & Growth & Removals & Inventory & Growth & Removats \\
\hline Percent & $\begin{array}{l}\text { Thousand } \\
\text { acres }\end{array}$ & \multicolumn{3}{|c|}{ - - - Million cubic feet- . - } & \multicolumn{3}{|c|}{ - - -2/Million board feet- - - - } \\
\hline $\begin{array}{r}1 \\
2 \\
3 \\
4 \\
5 \\
10 \\
15 \\
20 \\
25 \\
50 \\
100\end{array}$ & $\begin{array}{r}2,131.6 \\
532.9 \\
237.2 \\
133.2 \\
85.3 \\
21.3 \\
9.4 \\
5.3 \\
3.4 \\
0.8 \\
0.2\end{array}$ & $\begin{array}{r}12,460.9 \\
3,115.2 \\
1,385.3 \\
779.7 \\
498.4 \\
124.6 \\
55.2 \\
31.3 \\
19.9 \\
5.0 \\
1.2\end{array}$ & $\begin{array}{r}705.6 \\
176.4 \\
78.4 \\
44.1 \\
28.2 \\
7.1 \\
3.1 \\
1.8 \\
1.2 \\
0.3 \\
0.1\end{array}$ & $\begin{array}{r}5,500.0 \\
1,375.0 \\
611.0 \\
343.7 \\
220.0 \\
55.0 \\
24.4 \\
13.7 \\
8.8 \\
2.2 \\
0.5\end{array}$ & $\begin{array}{r}84,456.1 \\
21,114.0 \\
9,382.0 \\
5,280.8 \\
3,378.2 \\
844.6 \\
375.8 \\
211.6 \\
135.4 \\
33.9 \\
8.5\end{array}$ & $\begin{array}{r}3,312.4 \\
828.1 \\
368.5 \\
207.0 \\
132.5 \\
33.1 \\
14.6 \\
8.3 \\
5.3 \\
1.3 \\
0.3\end{array}$ & $\begin{array}{r}8,750.1 \\
2,187.5 \\
972.2 \\
546.9 \\
350.0 \\
87.5 \\
38.9 \\
21.9 \\
14.0 \\
3.5 \\
0.9\end{array}$ \\
\hline
\end{tabular}

$1 /$ At the 68-percent probability level.

2/International $1 / 4$-inch rule. 

Jakes, Pamela J.

1980. Minnesta forest statistics, 1977. U.S. Department of Agriculture Forest Service, Resource Bulletin NC-53, 85 p. U.S. Department of Agriculture Forest Service, North Central Forest Experiment Station, St. Paul, Minnesota.

Presents highlights and statistics from the Fourth Minnesota Forest Inventory. Includes detailed tables of forest area, timber volume, net annual growth, timber removals, mortality, and timber products output.

KEY WORDS: forest inventory, forest area, timber volume, timber growth, timber removals.

Jakes, Pamela J.

1980. Minnesta forest statistics, 1977. U.S. Department of Agriculture Forest Service, Resource Bulletin NC-53, 85 p. U.S. Department of Agriculture Forest Service, North Central Forest Experiment Station, St. Paul, Minnesota.

Presents highlights and statistics from the Fourth Minnesota Forest Inventory. Includes detailed tables of forest area, timber volume, net annual growth, timber removals, mortality, and timber products output.

KEY WORDS: forest inventory, forest area, timber volume, timber growth, timber removals. 
